This document is the Accepted Manuscript version of a Published Work that appeared in final form in Macromolecules 53(12) : 4581-4604 (2020) copyright (c 2020 American Chemical Society after peer review and technical editing by the publisher. To access the final edited and published work see https://doi.org/10.1021/ acs.macromol.0c00223

\title{
Self-nucleation Effects on Polymer Crystallization
}

\author{
Leire Sangroniz ${ }^{1}$, Dario Cavallo ${ }^{2 *}$ and Alejandro J. Müller ${ }^{1,3^{*}}$
}

${ }^{1}$ POLYMAT and Polymer Science and Technology Department, Faculty of Chemistry, University of the Basque Country UPV/EHU, Paseo Manuel de Lardizábal, 3, 20018 Donostia-San Sebastián, Spain.

${ }^{2}$ Department of Chemistry and Industrial Chemistry, University of Genova, via Dodecaneso, 31 - 16146 Genova, Italy

${ }^{3}$ IKERBASQUE, Basque Foundation for Science, Bilbao, Spain.

*Corresponding authors: dario.cavallo@unige.it; alejandrojesus.muller@ehu.es 


\begin{abstract}
The existence of a "memory" of the previous crystalline state, which survives melting and enhances re-crystallization kinetics by a self-nucleation process, is wellknown in polymer crystallization studies. Despite being extensively investigated, since the early days of polymer crystallization studies, a complete understanding of melt memory effects is still lacking. In particular, the exact constitution of self-nuclei is still under debate. In this perspective, we provide a comprehensive and critical overview of melt memory effects in polymer crystallization. After the phenomenology of the process and some key concepts are introduced, the main experimental results of the last decades are summarized. Analogies and discrepancies of the melt memory characteristics of different polymeric systems are highlighted. Based on this background, the most significant interpretations and theories of melt memory effects are described; underlining that different interpretations may apply to various specific cases. Recent insights on self-nucleation, gained thanks to a multi-technique approach (combining calorimetry, rheology, infrared and dielectric spectroscopy), are presented. The role of intra/inter-chain segmental contacts in the strength of melt memory effects, and the differences between homopolymers and copolymers behavior, are discussed. Finally, we identify areas where further research in the field is needed to shed light on the longstanding questions regarding the origin of melt memory effects in semi-crystalline polymers.
\end{abstract}

Keywords: Melt memory; self-seeds; self-nucleation; self-nuclei; polymer crystallization. 


\section{Index}

1. Introduction and phenomenology of self-nucleation and melt memory effects......... 4

1.1. Homogeneous and heterogeneous nucleation ..................................................... 4

1.2. Melt memory as a special case of homogeneous nucleation................................... 7

1.3. Self-nucleation and self-seeding in polymer crystallization ................................. 11

2. Effect of experimental variables on melt memory ................................................. 18

2.1. Effect of self-nucleation temperature on crystalline morphology and structure .. 18

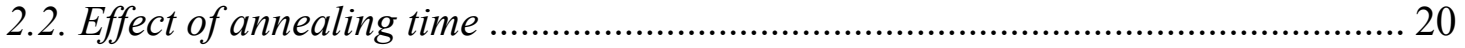

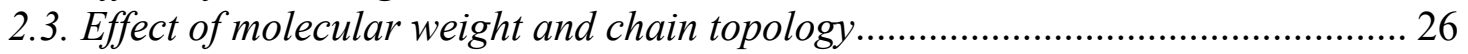

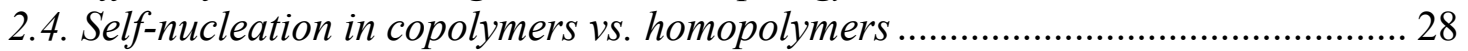

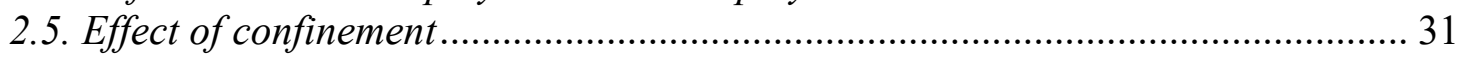

3. Interpretation(s) of melt memory effects ........................................................... 34

4. Recent experiments with combined techniques: the role of intermolecular

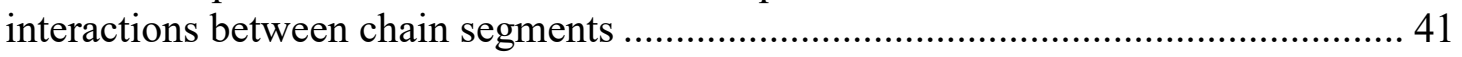

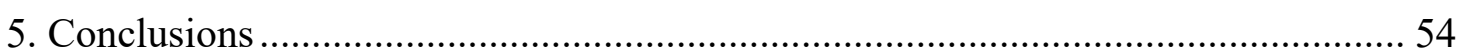

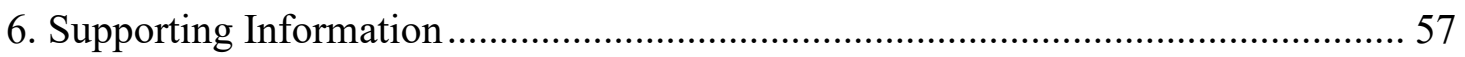



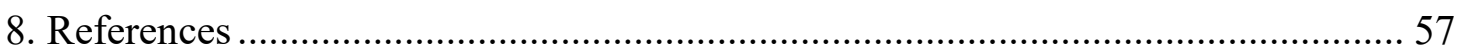




\section{Introduction and phenomenology of self-nucleation and melt memory}

effects

\subsection{Homogeneous and heterogeneous nucleation}

Polymer nucleation can, in principle, proceed by spontaneous aggregation of chain segments to form homogeneous nuclei. This homogeneous nucleation involves the production of new surfaces and results in a high energy barrier that requires very large supercooling to be surpassed ${ }^{1-3}$. Hence, homogeneous nucleation does not often occur in bulk polymers at conventional cooling time scales.

Polymer chains prefer to nucleate on pre-existing surfaces, a process that requires a significantly lower energy barrier and much lower supercoolings. This process is known as heterogeneous nucleation and occurs in bulk polymers since they normally contain a substantial amount of heterogeneities, such as catalytic debris, impurities or additives ${ }^{1-6}$.

When a bulk polymer sample is divided into a series of small domains whose number is larger than the number of efficient heterogeneities that are contained within the polymer, a certain number of domains without any active heterogeneities will be produced. This is the principle behind the classic "droplet experiments" performed in metals $^{7-10}$, alkanes ${ }^{11}$ and polymers ${ }^{4,12-15}$ to study homogeneous nucleation.

Droplet experiments have been recently revisited by Dalnoki-Veress et al. ${ }^{16-20}$. In one example ${ }^{16}$, they spin-coated poly(ethylene oxide), PEO, on a polystyrene, PS, substrate and then they annealed the sample. In this way, PEO is dewetted from the PS substrate, thereby producing an ensemble of PEO droplets. Figure 1a shows results from Massa and Dalnoki-Veress ${ }^{17}$, where they have followed the fraction of solidified droplets as they cooled a sample that is composed of clean PEO droplets produced by dewetting. The droplets that have crystallized are easy to recognize by their birefringence (they appeared as bright white dots in Figure 1a) in comparison to the amorphous dark droplets (see inset). Clean PEO droplets crystallized in the range of -2 until $-7^{\circ} \mathrm{C}$ upon cooling from the melt. For bulk PEO or for droplets with active heterogeneities, the crystallization temperature range was $56-46^{\circ} \mathrm{C}$. Moreover, performing isothermal experiments, Dalnoki-Veress et al. ${ }^{16-20}$ found that for larger droplets, the nucleation rate was faster, and it scaled with the third power of the radius 
of the droplet. This confirmed that the homogeneous nucleation occurs inside the volume of the PEO droplets, and also that surface nucleation was not observed.

a)

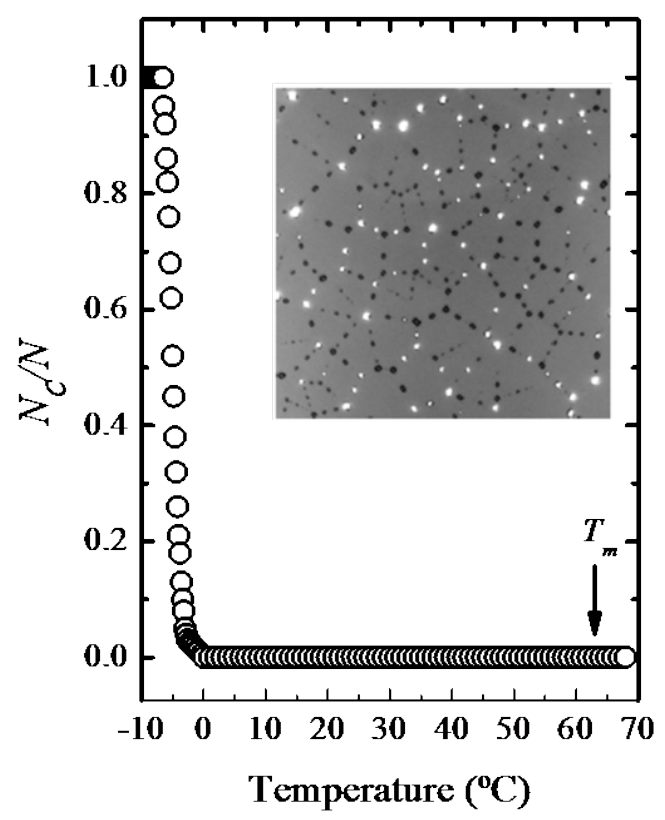

b)

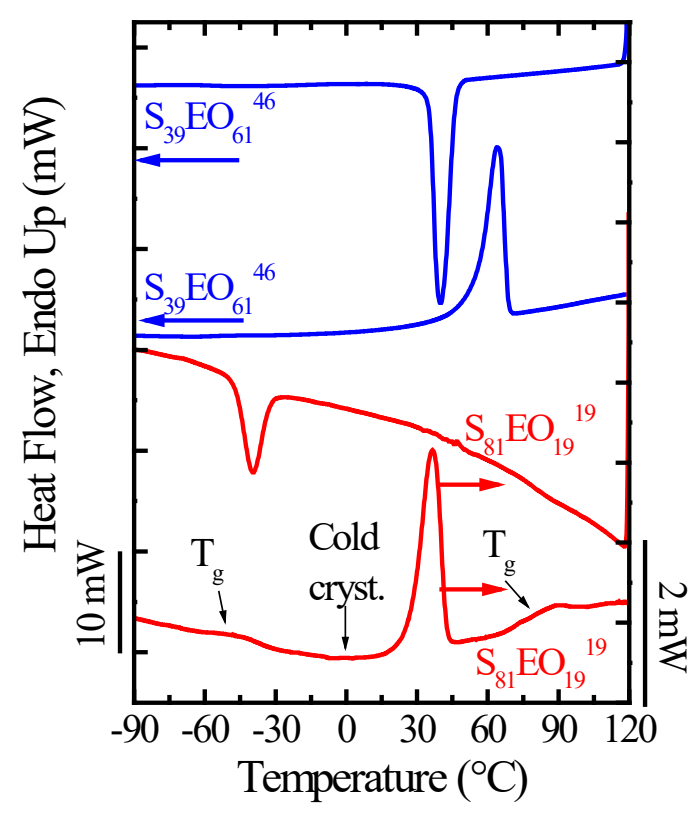

c)

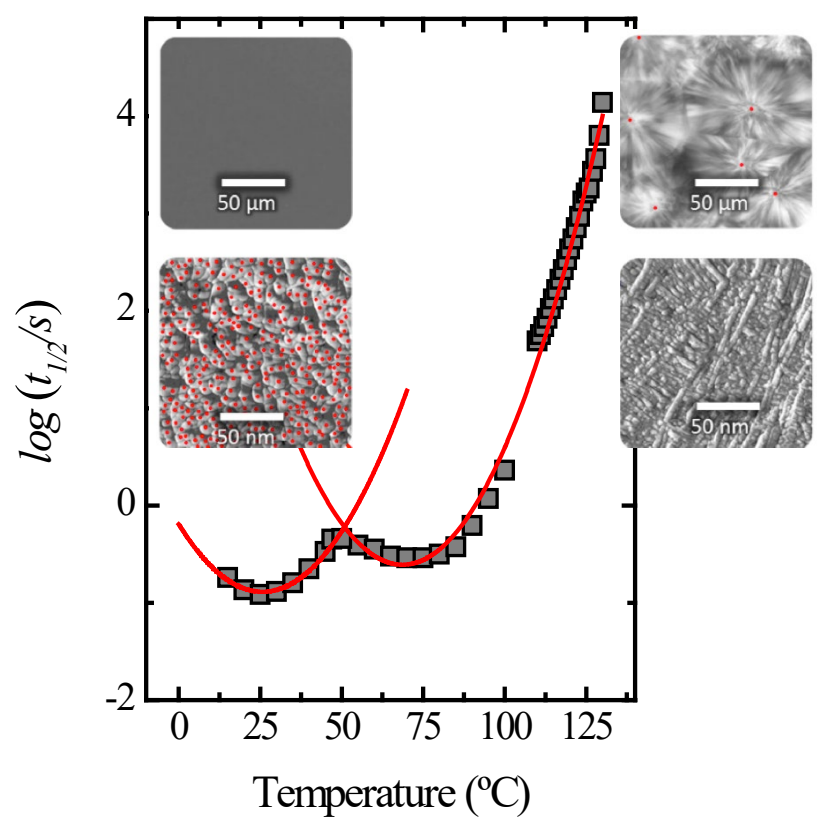

Figure 1. Examples of homogeneous nucleation: a) Polarized light optical micrograph of a section of a PEO droplet dispersion $(1000 \mu \mathrm{m}$ wide $)$ taken at $T_{c}=-2.6^{\circ} \mathrm{C}$. Amorphous droplets appear dark, whereas semi-crystalline droplets appear white under crossed polarizers. The plot shows the fraction of crystallized droplets as a function of temperature upon cooling. Reprinted with permission from ref. 17. Copyright 2004 APS Physics; b) Cooling and heating DSC scans $\left(10^{\circ} \mathrm{C} / \mathrm{min}\right)$ for PS-b-PEO diblock 
copolymers of different compositions (see text). Heterogeneous and homogeneous nucleation give rise to very different supercoolings, see the large differences in peak crystallization temperatures. The difference in melting points is due to the difference in molecular weights between the two PEO blocks. Adapted with permission from ref. 28; c) Crystallization half-times of iPP at low (heterogeneous nucleation) and high supercooling (homogeneous nucleation). Images in the inset show morphologies obtained in the two distinct nucleation regimes (top image optical microscopy, bottom image AFM), with the red dots indicating nuclei position. Left images: homogeneous nucleation; right images: heterogeneous nucleation. Adapted with permission from ref. 36 Copyright 2017 IOP Publishing.

Other convenient ways to subdivide a bulk polymer into individual droplets are the use of immiscible polymer blends, ${ }^{21-23}$ block copolymer microphase separated microdomains ${ }^{23-33}$, or polymers infiltrated in alumina templates ${ }^{23,32-34}$. In some of these cases, much smaller microdomain (or nanodomain) sizes can be obtained.

One example is provided in Figure $1 \mathrm{~b}$, where changing the composition of strongly segregated PS- $b$-PEO diblock copolymers can change the morphology, and concomitantly the nucleation mechanism of the PEO microphase separated domains. In copolymer $\mathrm{S}_{39} \mathrm{EO}_{61}{ }^{46}$ (the subscripts give the composition in wt.\% and the superscript the number average molecular weight of the entire copolymer, $M_{n}$, in $\mathrm{kg} / \mathrm{mol}$ ), the PEO phase forms a matrix where PS cylinders are dispersed. As the PEO phase constitutes a percolated matrix, the crystallization starts from heterogeneous nuclei with an exothermic peak at $37^{\circ} \mathrm{C}$, similar to that of bulk $\mathrm{PEO}^{28}$. However, when the block copolymer only contains $19 \%$ PEO, the morphology changes to a PS matrix filled with PEO nanospheres of approximately $20 \mathrm{~nm}$ in average diameter. The crystallization of these nanodroplets occurs at extreme supercoolings, i.e., around -30 to $-50{ }^{\circ} \mathrm{C}$, very close to PEO glass transition temperature (i.e., at circa $-60{ }^{\circ} \mathrm{C}$ ). Since the number of droplets greatly exceeds the number of heterogeneities typically present in bulk PEO (about $10^{15}$ droplets $/ \mathrm{cm}^{3}$ vs. ca. $10^{6}$ heterogeneities $/ \mathrm{cm}^{3}$ ), the majority of nanodroplets can be considered clean. Therefore, the crystallization must have started within the droplets by bulk homogeneous nucleation. We note that the differences in nonisothermal crystallization temperature of the same polymer (PEO) in dewetted droplet or confined nanodomains of block-copolymers (Figure 1a and b) is attributed to the difference in the domain volume, another characteristic of homogeneous nucleation ${ }^{29}$.

Another condition under which homogeneous nucleation can be observed is crystallization at very large supercoolings. Schick et al. ${ }^{35,36}$ have determined overall crystallization kinetics of several polymers in such temperature range employing fast 
scanning calorimetry at cooling rates as large as 50.000 to $500.000 \mathrm{~K} / \mathrm{s}$. Their results show that at crystallization temperatures relatively close to the glass transition, the kinetics can be completely dominated by homogeneous nucleation even in bulk polymers that contain heterogeneities. The occurrence of homogeneous nucleation is manifested by a distinct change of crystallization kinetics and semi-crystalline morphology (see Figure 1c). ${ }^{35,36}$

\subsection{Melt memory as a special case of homogeneous nucleation}

The existence of memory effects has been experimentally observed for decades in quiescent polymer crystallization ${ }^{18,37-65}$. When a semi-crystalline bulk polymer is heated to temperatures well above the equilibrium melting point of the material $\left(T_{m}{ }^{0}\right)$ for long enough times (e.g., a few minutes), it is expected to melt and achieve an isotropic melt state. In the context of this perspective, the term "isotropic" does not refer to chain orientation, but it is used to describe a "fully relaxed" or "equilibrium" melt. This term is preferred here with respect to the often used "homogeneous", in order to avoid possible confusion with the notion of "homogeneous nucleation". If this isotropic melt is subsequently cooled in a DSC, the sample will crystallize, and the nonisothermal crystallization exotherm observed will be reproducible and will always occur at the same peak crystallization temperature $\left(T_{c}\right)$ (with some small variations due to the experimental error of the DSC technique which are in general smaller than $1{ }^{\circ} \mathrm{C}$ ), if the experimental conditions are kept constant. In this case, the material nucleates on existing heterogeneities which are temperature resistant (in the temperature range employed) and the peak crystallization temperature is a function of the nucleation density in the polymer sample.

Now let's consider the case when this semi-crystalline polymer sample is heated to a temperature (which for convenience we shall term $T_{s}$ ) in between the experimentally observed melting point, $T_{m}$, and the equilibrium melting temperature, for a fixed amount of time (e.g., $5 \mathrm{~min}$ ). As $T_{s}$, in this case, is higher than $T_{m}$ ( $T_{m}$ being the temperature at which the endothermic melting peak completely disappears in a DSC heating scan), the polymer should not contain any crystal fragments that could act as self-seeds. Nevertheless, it is frequently observed (depending on the value of $T_{s}$ ) that upon cooling from the melt, the sample crystallizes at temperatures higher than the previous case (i.e., when the thermal history is completely erased), indicating that a selfnucleation process has occurred. The lower the value of $T_{s}$, the higher is the $T_{c}$ upon 
cooling from the melt, indicating a stronger nucleation effect. This peculiar nucleation effect has been termed in different ways in the literature: crystalline memory ${ }^{38,39,41,56-}$ $58,61,63,66-80$, melt memory $49,52,60,64,81-94$, in some works both terms are used indistinctly ${ }^{18,47,48,62,65,95-98}$ and in other cases a more general term, memory effect, has been employed ${ }^{43,45,51,53,54,55,59,99-119}$. In this perspective, we will use the term melt memory to emphasize that this effect occurs when according to calorimetric evidence, there are no crystal fragments present in the melt (i.e., in the so-called Domain IIa, see section 1.3 below).

These extra nuclei produced by melt memory (or self-nuclei) cannot be heterogeneous in origin, as the heterogeneous nuclei in the sample can only generate a limited amount of crystal nuclei, this is reflected in the constant value of $T_{c}$ obtained when the sample is cooled from the isotropic melt. In fact, the additional self-nuclei generated by the melt memory effects arise from the "structure" of the non-isotropic melt, and therefore must be considered as a special case of homogeneous nucleation, as these extra nuclei are formed by aggregation of polymeric segments in the nonisotropic melt.

We again specify that the term "non-isotropic" in this context does not refer to any particular polymer orientation or anisotropy, but rather to a "heterogeneous" structure. We prefer to avoid the term "heterogeneous" melt in order not to generate possible confusion with heterogeneous nucleation. Such "structure" of the non-isotropic melt is not easy to ascertain with precision. As it will be discussed in more detail below, this melt structure has been hypothesized as being a residual orientation of the chains coming from the original macromolecular conformations in the crystal ${ }^{47,58,62,63,87,120}$ or produced by sticky interactions of the chains in the melt, arising from inter-chain segmental contacts, such as inter/intra molecular hydrogen bonds or other types of weak forces $^{61,63-65,97}$.

Finally, if $T_{s}$ is lower than $T_{m}$, crystal fragments remain unmolten in the sample and constitute ideal heterogeneous nuclei, obviously providing a perfect lattice matching with the crystallizing material, that we shall term self-seeds.

Experimental observations that demonstrated the effects of the employed $T_{S}$ values on polymer crystal nucleation have been reported in the literature since the early days of polymer crystallization studies ${ }^{37-40,66-68,121-127}$. Figure 2 a replots data reported by Banks and Sharples more than 50 years $\mathrm{ago}^{37}$. They studied the nucleation and crystallization of a poly(ethylene oxide) sample by polarized light optical microscopy 
(PLOM) and dilatometry. The sample was isothermally crystallized from the melt at $T_{c}=52.7^{\circ} \mathrm{C}$, then the sample was heated up to a selected $T_{s}$ temperature for $10 \mathrm{~min}$ and later cooled to the same isothermal crystallization temperature, at which the number of nucleation sites was counted.

Figure $2 \mathrm{a}$ plots the number of nuclei observed in the microscope field (corresponding to a volume of $7.5 \times 10^{-6} \mathrm{~cm}^{3}$ ) as a function of $T_{s}$ temperature ${ }^{37}$. There is a very large increase in nucleation density, as $T_{s}$ is reduced. At the time, even when the effect of the melting temperature on the subsequent nucleation was recognized and indirectly related to some kind of memory, the nucleation was thought to be always heterogeneous. The authors explained their results by considering that impurities were present in the polymer containing crevices in which the polymer could crystallize to produce self-seeds with increased melting points, a speculation made earlier by Price ${ }^{122}$. The first data point was taken at a $T_{s}$ temperature of $66.2^{\circ} \mathrm{C}$, which is the experimental melting point of the sample determined by dilatometry.

a)



b)

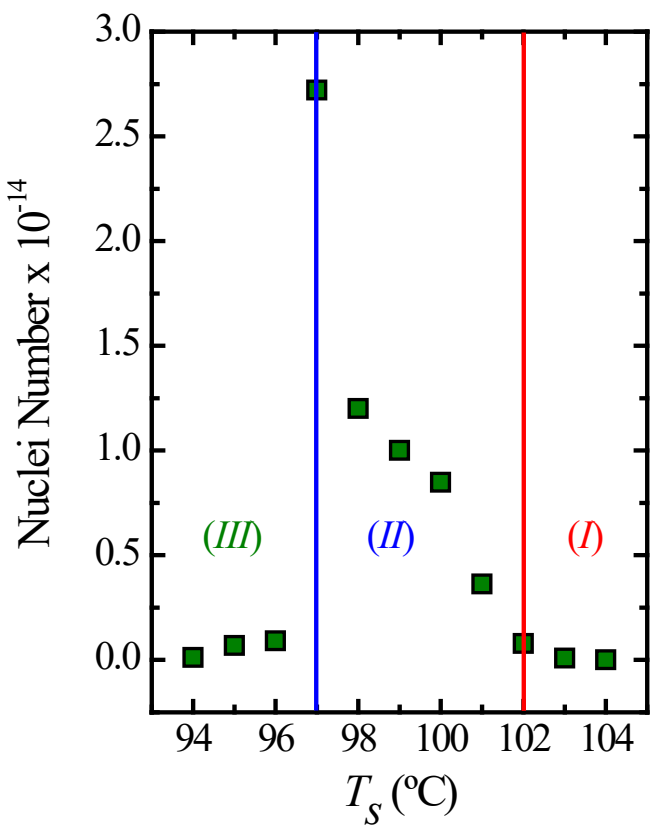

Figure 2. a) Effect of melt temperature on nucleation density observed by polarized light optical microscopy in PEO previously crystallized at $52.7^{\circ} \mathrm{C}$. Data replotted from the work of Banks and Sharples, ref 37 . The y axis represents the number of nuclei observed in a viewed volume of $7.5 \times 10^{-6} \mathrm{~cm}^{3}$. The three regions in the plot marked as $(I)$, (II) and (III) separated by vertical lines, are explained in the text. b) Effect of dissolution temperature on nucleation density measured by electron microscopy 
techniques in PE xylene solution (0.01\%). Data replotted from the work of Blundell and Keller, ref. 39. In the $y$ axis the number of nuclei per gram of polymer is represented.

The data presented in Figure 2a can now be explained, with the hindsight of recent studies on melt memory. The three different cases explained can be identified in Figure 2a from high to low temperatures:

(I) Behavior dominated by heterogeneous nucleation produced by impurities or pre-existing heterogeneities. At temperatures higher than $90^{\circ} \mathrm{C}$, the nucleation density becomes low and constant, corresponding to the common case of heterogeneous nucleation (coming from existing heterogeneities in the bulk polymer) when cooling the polymer from an isotropic melt.

(II) Behavior dominated by homogeneous nucleation produced by melt memory (self-nucleation). At lower temperatures, in between 70 and $90^{\circ} \mathrm{C}$, the nucleation density strongly depends on $T_{s}$ temperature and increases as $T_{s}$ decreases, indicating that a melt memory process is present. This additional nucleation is occurring from selfnuclei present in the non-isotropic melt, a case of peculiar homogeneous nucleation that occurs in structured or self-nucleated melts.

(III) Behavior dominated by heterogeneous nucleation produced by self-seeds. At $T_{s}$ values of $66.2^{\circ} \mathrm{C}$ and lower, self-seeding occurs (i.e., crystals fragments will be present) and the nucleation density increased so much that Banks and Sharples were not able to determine it quantitatively (it is represented schematically as a dotted line in Figure 2a).

Blundell et al. ${ }^{38}$ reported similar findings when studying the crystallization of single crystals in solution. They denoted the effects they measured as a new selfnucleation phenomenon. They found that the crystallization time could be substantially shortened if the dissolution of a standard suspension of polyethylene crystals in xylene was carried out below a specific temperature, which they denoted $T_{f}$. The crystallization rate in solution decreased with the increase of $T_{s}$ (the dissolution temperature, where $T_{s}$ $<T_{f}$ ), and the effect disappeared at $T_{f}$.

In a follow-up publication, Blundell and $\mathrm{Keller}^{39}$ studied the crystallization of linear polyethylene (PE) in solution. First, the polymer was completely dissolved in xylene, and then it was cooled down until $80^{\circ} \mathrm{C}$ to obtain PE solution grown crystals. These PE crystals were reheated to a $T_{\mathrm{s}}$ temperature. The effect of heating rate, the time spent at $T_{\mathrm{s}}$ temperature and the value of $T_{\mathrm{s}}$ temperature on the nuclei number were 
studied. Microscopy techniques were employed to analyze the number of nuclei produced at different conditions.

In Figure $2 b$, the data obtained by Blundell and Keller are shown by plotting the number of nuclei per gram of polymer as a function of dissolution temperature. As in the case of PEO (Figure 2a), at temperatures above $102^{\circ} \mathrm{C}$ (i.e., temperature region labeled I), the number of nuclei does not change. At temperatures between 97 and $102^{\circ} \mathrm{C}$ the nucleation density increases upon reducing the $T_{\mathrm{s}}$ temperature due to the presence of self-nuclei which apparently were not completely dissolved, i.e., temperature region labeled II. At temperatures below $97^{\circ} \mathrm{C}$, there is some peculiar behavior in the nucleation density; in fact, a significant reduction of the nuclei number is observed, i.e., temperature region labeled III. This behavior can be explained considering that in this temperature region the polymer is not completely dissolved.

\subsection{Self-nucleation and self-seeding in polymer crystallization}

The terms self-seeding and self-nucleation have been used indistinctively in polymer nucleation studies in the past. However, we propose that a clear distinction should be made to clarify the nomenclature related to melt memory effects.

Self-seeding is the process that leads to the production of seeds or crystal fragments that can act as nuclei. Although many early studies had noticed that incomplete polymer melting could generate self-seeding $37-40,66-68,121-128$, it was not until the work of Blundell et al. ${ }^{38}$ that this process was systematically studied ${ }^{128}$. In this early reference, the authors employed the self-seeding technique to control the nucleation process and the size of polyethylene single crystals grown from solution. Later, Fillon et al. ${ }^{43}$ developed a DSC based thermal protocol to apply similar concepts on meltcrystallized isotactic polypropylene. They employed a very large temperature range encompassing the three different cases described in Figure 2 above. They referred to their study as self-nucleation (SN). 


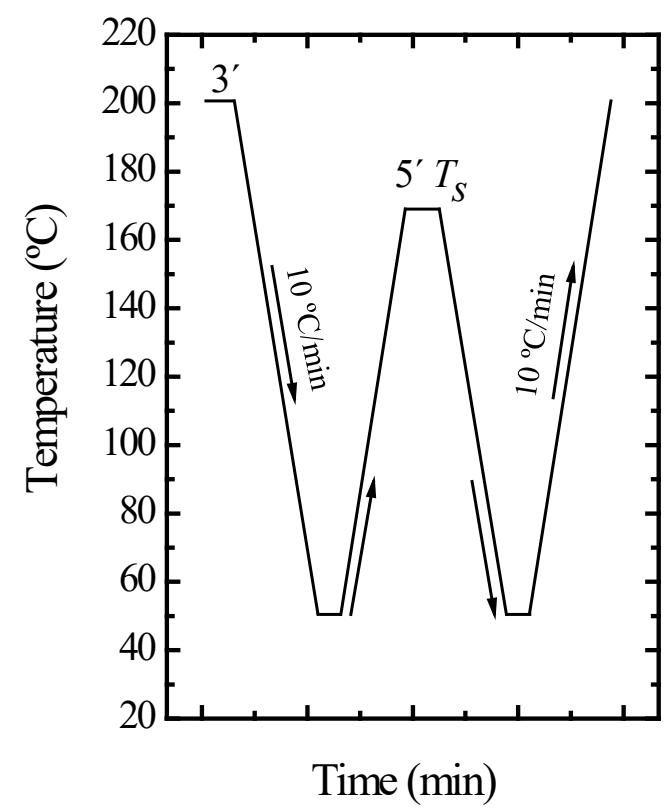

Figure 3. Schematic representation of a self-nucleation (SN) experiment ${ }^{43}$.

We shall use the term self-nucleation (SN) in the general sense of applying a thermal protocol (like the one shown in Figure 3) to study polymer nucleation including a self-seeding temperature range, a melt memory temperature range and a heterogeneous nucleation temperature range, depending on the applied temperature and thermal history. Polymer self-nucleation employing DSC has been recently reviewed by Michell et al. ${ }^{62}$.

Figure 3 illustrates the typical SN experimental protocol applied by DSC experiments. The standard procedure consists of 5 steps performed at a constant scan rate (e.g., $\left.10^{\circ} \mathrm{C} / \mathrm{min}\right)$ :

(1) Erasing melt memory by heating up to $25-30^{\circ} \mathrm{C}$ above the peak melting temperature for $3 \mathrm{~min}$. This treatment is generally effective in erasing all thermal history and produce an isotropic melt in most homopolymer samples.

(2) Producing a standard thermal history by cooling from the isotropic melt to a low temperature (at which the material has finished the non-isothermal crystallization process). The peak crystallization temperature obtained during this DSC cooling run constitutes the standard crystallization temperature (or standard $T_{c}$ ), and it is proportional to heterogeneity density present in the polymer sample. 
(3) Heating to a temperature that we denote $T_{s}$. The sample is kept at this $T_{s}$ value for 5 minutes.

(4) Cooling from $T_{s}$ to the minimum temperature at which the sample has finished its non-isothermal crystallization process.

(5) Heating to the melting temperature needed to erase crystalline history, as established in step 1.

Three self-nucleation Domains are defined by inspecting the DSC scans during the cooling and subsequent heating runs ${ }^{43,62}$. They critically depend on the value of $T_{s}$ applied during step (3) above.

The polymer sample is in Domain I or isotropic melt Domain if $T_{S}$ is high enough to completely melt the polymer and produce an isotropic melt where only hightemperature resistant heterogeneities remain.

Domain II or self-nucleation Domain occurs when $T_{s}$ is high enough to: (a) melt the majority of the polymer crystals but low enough to leave some crystal fragments that act as self-seeds in the lower part of Domain II or self-seeding subDomain (Domain IIb); or (b) melt the polymer crystals but without erasing its melt memory, thereby creating self-nuclei (not self-seeds) in the higher temperature range of Domain II or melt memory sub-Domain (Domain IIa).

If the $T_{s}$ value is lower than a certain temperature, it will only produce partial melting. In this case, the sample will be self-seeded and additionally, the crystals that did not melt will be annealed, and the polymer will be in Domain III or self-nucleation and annealing Domain (or self-seeding and annealing Domain).

To illustrate the application of the self-nucleation protocol, we have chosen poly(butylene succinate), PBS, as an example, as it displays all 3 self-nucleation Domains, including a pronounced melt memory sub-Domain IIa. 
a)

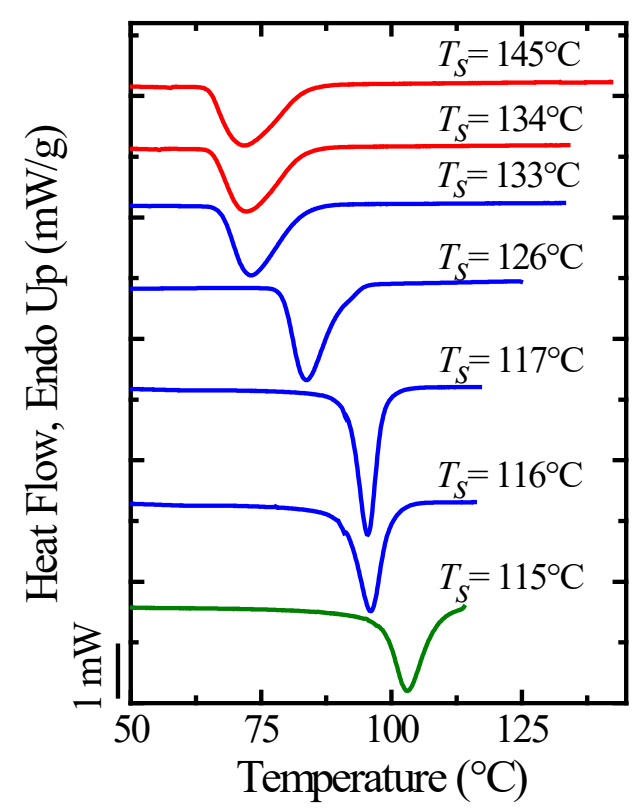

b)

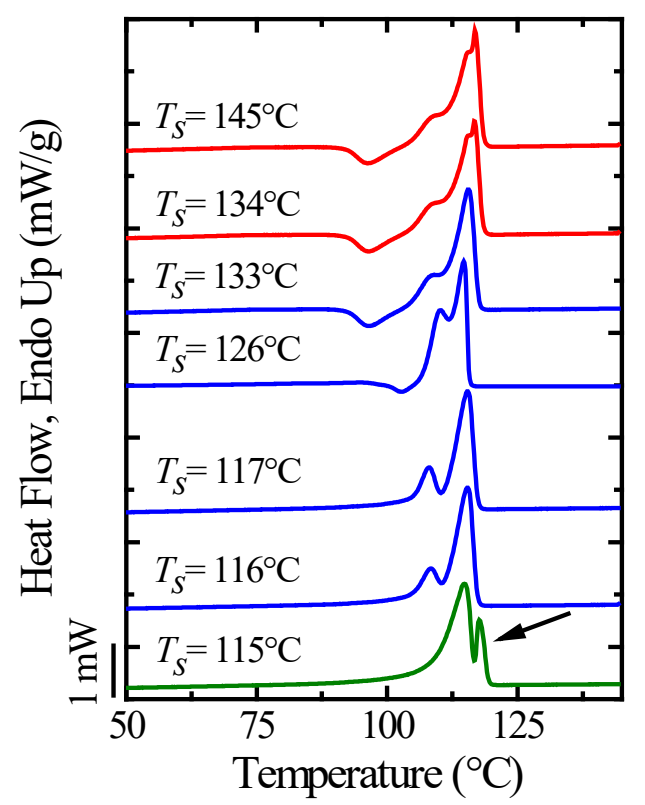

Figure 4. Self-nucleation of PBS: a) DSC cooling scans from the indicated self-nucleation $\left(T_{s}\right)$ temperatures and b) subsequent heating scans at $10^{\circ} \mathrm{C} / \mathrm{min}$ from ref. 57 .

Figure 4a shows DSC cooling runs from selected $T_{s}$ values. At $T_{s} \geq 134^{\circ} \mathrm{C}$, the melt memory is completely erased, and the material is in Domain I or complete melting Domain. The peak crystallization temperature is constant in Figure $4 \mathrm{a}$ for these $T_{s}$ temperatures (as the number of temperature resistant existing heterogeneities in the bulk polymer is also constant) and the melting traces in Figure $4 \mathrm{~b}$ are invariant upon subsequent heating. The DSC scans corresponding to Domain I have been drawn in red in the digital version of this paper. This is the typical behavior of a bulk polymer crystallizing by heterogeneous nucleation.

PBS experiences only self-nucleation when $T_{s}$ temperatures lower than $134^{\circ} \mathrm{C}$, but higher than or equal to $116^{\circ} \mathrm{C}$, are employed, and its crystallization temperature correspondingly increases (Figure $4 \mathrm{a}$ ). The maximum peak melting points in Figure $4 \mathrm{~b}$ do not show any signs of annealing. The sample is in the exclusive self-nucleation Domain (i.e., Domain II). A blue color code has been used to denote Domain II in Figure 4 (see the digital version of the paper). As $T_{s}$ temperature decreases, the cold crystallization process in the subsequent melting of PBS disappears, as the sample can increase its crystallinity degree during cooling because of the enhanced nucleation.

Finally, when $T_{s} \leq 115^{\circ} \mathrm{C}$ is employed, the melting is only partial and the unmolten crystals thicken (i.e. anneal) during the $5 \mathrm{~min}$ holding time at $T_{s}$, the sample is 
then in the self-nucleation and annealing Domain (Domain III) or self-seeding and annealing Domain. The appearance of a high temperature additional melting peak (see Figure $4 \mathrm{~b}$, heating scan after SN at $T_{S}=115^{\circ} \mathrm{C}$ ) is a signature of Domain III, as signaled with an arrow in Figure 4b. The DSC curves in Domain III are plotted in green in the digital version of the paper.



Figure 5. A plot of the self-nucleation Domains for PBS homopolymer (vertical lines) on top of the standard DSC melting trace (with color codes for the digital version of the article, green: DIII, blue: DII and red: DI). Insets include PLOM micrographs taken during cooling from $T_{s}=145^{\circ} \mathrm{C}$ (Domain $I$ ) and $T_{S}=116^{\circ} \mathrm{C}$ (Domain II). The data points represent peak crystallization temperatures obtained after SN (plotted on the right-hand side y-axis) as a function of $T_{s}$ values (plotted on the x-axis). Notice the solid vertical lines dividing the three different self-nucleation Domains. A segmented vertical line indicates the division of Domain II into two sub-Domains: at low temperatures, the narrow self-seeding sub-Domain $I I b$ (where crystal fragments act as self-seeds) and at high temperatures, the much wider melt memory sub-Domain IIa exhibited by PBS (where the $T_{s}$ temperature is higher than the end of the melting endotherm). Modified figure from ref. 57.

Figure 5 shows a summary of the $\mathrm{SN}$ results plotted in a single figure. The standard DSC melting scan of the PBS homopolymer has been plotted (i.e., the subsequent heating scan after the sample was cooled from the isotropic melt, Domain I, at $10^{\circ} \mathrm{C} / \mathrm{min}$ ) and vertical lines separate the three different self-nucleation Domains. Color codes are used (in the digital version of the paper) to indicate the Domains as in Figure 4: red for Domain I, blue for Domain II and green for Domain III. The changes in peak crystallization temperatures $\left(T_{c}\right)$ as a function of $T_{s}$ values have also been 
represented in Figure 5 (see data points referred to the right-hand side Y-axis with $T_{s}$ temperatures plotted in the $\mathrm{X}$-axis).

A significantly large increase in $T_{c}$ can be seen in Figure 5, as the sample crosses from Domain I to Domain II (going from right to left), which is directly related to the exponential increase in nucleation density caused by the newly created self-nuclei in Domain $I I^{43,62}$. Müller et al. have argued that Domain II can be divided into two subDomains $^{62,65}$, as indicated by a segmented vertical line. In the low-temperature part of Domain II (labeled DIIb), self-seeding is the origin of the increase in nucleation density as $T_{s}$ decreases, since crystal fragments (i.e., self-seeds) are present in this $T_{s}$ temperature range, as the polymer did not completely melt at temperatures between 116 and $118^{\circ} \mathrm{C}$. In DIIb crystal fragments surviving from partial melting can produce nucleation by homo-epitaxy.

It is remarkable how the high-temperature melt memory sub-Domain IIa (labeled DIIa in Figure 5) encompasses temperatures much higher than the end of PBS melting. The self-nuclei present in the temperature interval between 119 and $133^{\circ} \mathrm{C}$ cannot be crystalline as the temperature is too high. Hence, they must be regions in the self-nucleated melt with memory but without crystalline order. The nature of such selfnuclei will be discussed in detail below.

Two micrographs (obtained by PLOM) are shown in Figure 5 to illustrate the increase in the number of nuclei per unit area produced by self-nucleation. The micrographs were taken during cooling at $10^{\circ} \mathrm{C} / \mathrm{min}$, during step (4) of the $\mathrm{SN}$ protocol (see Figure 3 and its description above). The first one upon cooling from $T_{s}=145^{\circ} \mathrm{C}$ (Domain I) and the second from $T_{s}=116^{\circ} \mathrm{C}$ (Domain IIb). The self-nucleated sample (Domain IIb) contains a much larger number of smaller spherulites as compared to the sample cooled from Domain I.

Figure 6 presents a cartoon that summarizes and schematizes the SN Domains. Domain I is characterized by the presence of random coils (i.e., a way to represent the isotropic melt state). Self-nucleation occurs in Domain II, where two possibilities or cases are depicted in Figure 6. In the high-temperature range Domain II (or DIIa in Figure 5), some residual chain orientation remains in the melt (with ordered chain segment conformations similar to those in the crystals) as one possible way of representing melt memory (detailed discussion on the nature of the self-nuclei or melt structure will be given later in the text). 
In the low-temperature range Domain II (or DIIb in Figure 5), the situation is similar to that in DIIa but with additional small fragments of crystals (self-seeds) that do not anneal during the time spent at $T_{s}$. Finally, in Domain III the material is only partially melted, and the unmolten crystals thicken or anneal during the $5 \mathrm{~min}$ at $T_{s}$.

A final important definition can be made based on the above discussion and on Figure 6. From the melt memory point of view, it is important to define two types of melts. An isotropic melt is a relaxed melt obtained in Domain I after all thermal history and melt memory have been erased. On the other hand, the self-nucleated melt or nonisotropic melt is the melt that contains self-nuclei (and not self-seeds) of unknown origin (see the cartoon on the right-hand side of Figure 6 corresponding to the high $T_{s}$ DII or DIIa melt). These chains have neighboring segments possessing a residual correlation in the orientation (which have been drawn in the cartoon of Figure 6, corresponding to DIIa, in blue color) as they were once in the same crystal.

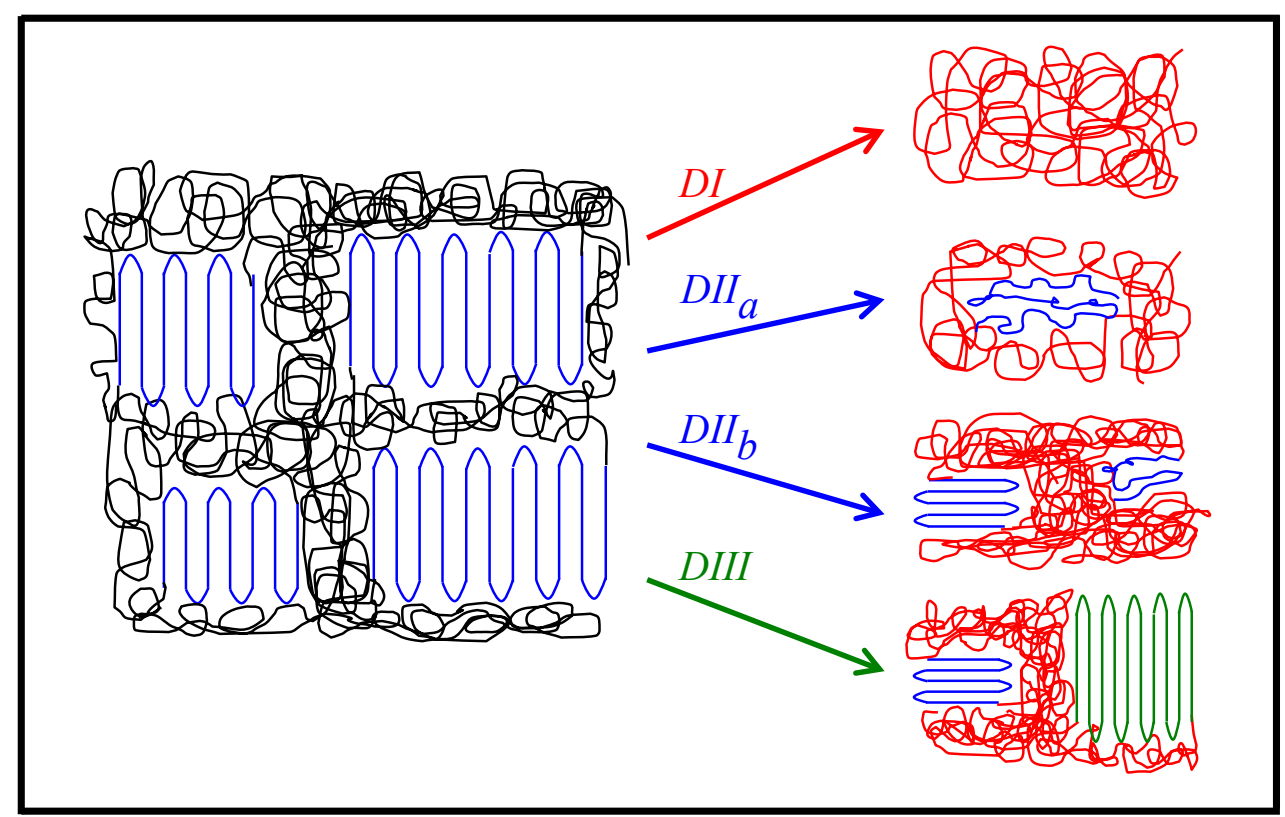

Figure 6. A schematic cartoon illustrating the different self-nucleation Domains. Based on ref. 62. Copyright 2017 Springer Nature.

We note that, so far, we only discussed melt-memory effects on crystallization upon cooling. Recently, it has been underlined that a partially ordered melt is also generated when crystals of low thermal stability are melted, and this structured meltstate is able to enhance the re-crystallization process upon heating, before final melting. 
The interested reader can find more detailed information in some recent publications ${ }^{129-}$ ${ }^{131}$, as this particular case of self-nucleation will not be discussed here. Moreover, nonequilibrium melt memory phenomena which are known to affect crystallization, such as those arising due to the relaxation of the effect of flow ${ }^{91,132,133}$, or to the existence of a transient partially disentangled melt topology ${ }^{134-136}$, are similarly not the focus of this Perspective article.

Therefore, after the essential concepts related to self-nucleation and memory effect have been outlined, the main experimental findings will be presented in the next section and used as a basis for the discussion of the main interpretations that have been proposed in the literature. Finally, the most recent results obtained by combining several characterization techniques will be summarized, and some directions for future research will be outlined.

\section{Effect of experimental variables on melt memory}

\subsection{Effect of self-nucleation temperature on crystalline morphology and}

\section{structure}

As outlined in section 1, self-nucleation greatly changes the morphology of the re-crystallized material. In general, the nucleation density and, consequently the final size of the spherulites, can be modified with this thermal procedure.

Lorenzo et al. $^{47}$ studied the morphology of isotactic polypropylene (iPP) employing polarized light optical microscopy (PLOM). They observed that for samples crystallized upon cooling from temperatures corresponding to Domain I, the morphology does not change since, in this temperature region, only temperature resistant heterogeneities remain in the sample, and nuclei form onto them at a given undercooling. When the self-nucleation temperature is reduced to temperatures corresponding to Domain II, an increase of the nucleation density and a reduction of the size of the spherulites is observed due to the presence of self-nuclei in DIIa or self-seeds in DIIb. At the temperature range corresponding to Domain IIb the size of the spherulites is really small and a granular morphology is obtained. Finally, in Domain III, according to the authors, a "phantom spherulite" morphological pattern is observed $^{43,47}$.

A detailed study of the morphology of iPP corresponding to each self-nucleation Domain has been performed by $\mathrm{Hu}$ et al. ${ }^{116}$. The authors have found that depending on 
the self-nucleation temperature, the size, birefringence, and the parent and daughter lamellae content (i.e., radial and tangential lamellae) varies. When the sample is crystallized upon cooling from a $T_{s}$ corresponding to Domain $I$, the spherulites contain radial and tangential lamellae. For samples crystallized in Domain II, the morphology changes from spherulites to granular, but the cross-hatched lamellar structure is preserved. Finally, for samples crystallized in Domain III, there are mainly radial lamellae and only a few tangential lamellae, resulting in negative birefringence ${ }^{116}$.

In Table S1 of the Supporting Information, the increase of the nucleation density obtained in the literature employing self-nucleation procedure is summarized for different polymers. A large variability between the different materials in the nucleation density increase can be noticed. In fact, the nucleation density of the self-nucleated samples can be from few times up to several orders of magnitude higher than the one obtained for heterogeneous nucleation from an isotropic melt. These differences could arise from the preparation method, the detection method and the thermal procedure (isothermal vs. non-isothermal crystallization). Although this spread in the measured nucleation density does not allow the identification of a common limiting value for the number of self-nuclei, it clearly indicates that the detectability of memory effect is highly dependent on the initial value of nucleating heterogeneities in the polymer.

On the other hand, the literature values of spherulite sizes or nucleation density suggest that the typical concentration of self-nuclei induced by SN at the ideal selfnucleation temperature within Domain IIb lays in the range of $10^{6}-10^{9} \mathrm{~cm}^{-3}$. Although the increase is meaningful, if compared to the common number of nucleating heterogeneities, it is still much smaller with respect of the number of nuclei that can be generated through homogeneous nucleation at much lower temperatures (larger supercoolings) by employing Fast Chip Calorimetry (of the order of $10^{15-18} \mathrm{~cm}^{-3} 36,137$ ). This difference may be related indeed to the larger supercoolings that can be applied by the ultra-fast cooling rates before nucleation can start. For instance, Fillon et al. ${ }^{43,100,101}$ reported for iPP an increase in nucleation density of one order of magnitude for each degree of reduction in $T_{s}$ temperature (equivalent to an increase in supercooling by $1^{\circ} \mathrm{C}$ ). Such results highlight the importance of supercooling also on self-nucleation. If the differences in supercooling could be neglected for the two cases (selfnucleated/homogeneous nucleated polymer), one could speculate that the striking difference in the generated number of nuclei may indicate that self-nucleation involves a 
relatively low number of molecules that conform the self-nuclei or a smaller number of crystal fragments that form the self-seeds.

In the case of polymorphic polymers, the content of the different crystalline phases can also vary, depending on the self-nucleation temperature. The effect of the self-nucleation thermal procedure on the content of polymorphism has been studied for several polymers, such as $\mathrm{iPP}^{43}$, poly(1-butene $)^{51,54,87}$, poly(amide 11$)^{45}$, poly(vinylidenefluoride ${ }^{85,119}$, poly(butylene adipate) $)^{73}$ among others ${ }^{138-140}$. The relevant literature is summarized in Table S2 of the Supporting Information. The interested reader is referred to the cited works since the extensive discussion of the role of polymorphism in self-nucleation is out of the main scope of this perspective article. However, since often the polymorphs of a given semi-crystalline polymer differ in chain conformation (e.g., extended vs. helical, etc...), the observed memory effect on polymorph selection, other than on re-crystallization kinetics, implies persistence in the melt of specific ordered conformers. In fact, a relation between self-nucleation and content of certain conformational bands in the melt, as measured via FT-IR, has been suggested in few cases $^{106,141}$.

\subsection{Effect of annealing time}

The determination of the different self-nucleation Domains critically depends on the self-nucleation temperature $\left(T_{s}\right)$, but it could also be dependent on the time spent at $T_{s}^{62}$. As it has been explained in Section 1, during the SN protocol employed to determine the different self-nucleation Domains, the values of $T_{s}$ are systematically varied, while the time spent at $T_{s}$ is maintained constant. Varying this time may affect the transition temperatures between Domains, as well as the width of Domain II. In the literature, the effect of annealing time at constant $T_{s}$ temperatures has been studied, although the results obtained are not always in agreement.

Fillon et al. ${ }^{43}$ studied the effect of annealing time on SN. The self-nucleation procedure was applied to iPP varying the time from $5 \mathrm{~min}$ to $20 \mathrm{~min}$, at a temperature corresponding to Domain II. However, the crystallization temperature upon subsequent cooling from $T_{s}$ did not change. They concluded that $\mathrm{SN}$ times longer than 5 min were unnecessary, as the produced self-nuclei saturated the total possible number of nuclei in the sample. 
Similar results have been found more recently with propene/ethylene copolymers $^{64}$, as maintaining the sample at $T_{s}$ temperatures within Domain IIb (i.e., the self-seeding Domain, where crystal fragments remain in the sample) for $1000 \mathrm{~min}$ did not change crystallization temperatures significantly. The effect of self-nucleation time on the crystallization temperature has also been studied with halogen-substituted polyethylenes $^{94}$. A $T_{s}$ temperature corresponding to Domain IIa was selected and long holding times at $T_{s}$ were employed (i.e., 600 to $1200 \mathrm{~min}$ ). No large variations of crystallization temperatures were observed.

However, different trends have also been reported in the literature. It has been observed that the number of nuclei can be reduced for some polymers when long times at $T_{S}$ temperatures within Domain II are applied. In most cases, the complete disappearance of the memory effects has not been observed.

Alfonso and Ziabicki ${ }^{142}$ studied iPP with different molecular weights focusing on the effect of the time spent at temperatures above melting on the subsequent crystallization kinetics. The thermal procedure employed was different than that usually applied in SN studies (see Section 1), as in their work, the sample was cooled down from $T_{s}$ until a constant temperature, at which isothermal crystallization kinetics was determined.

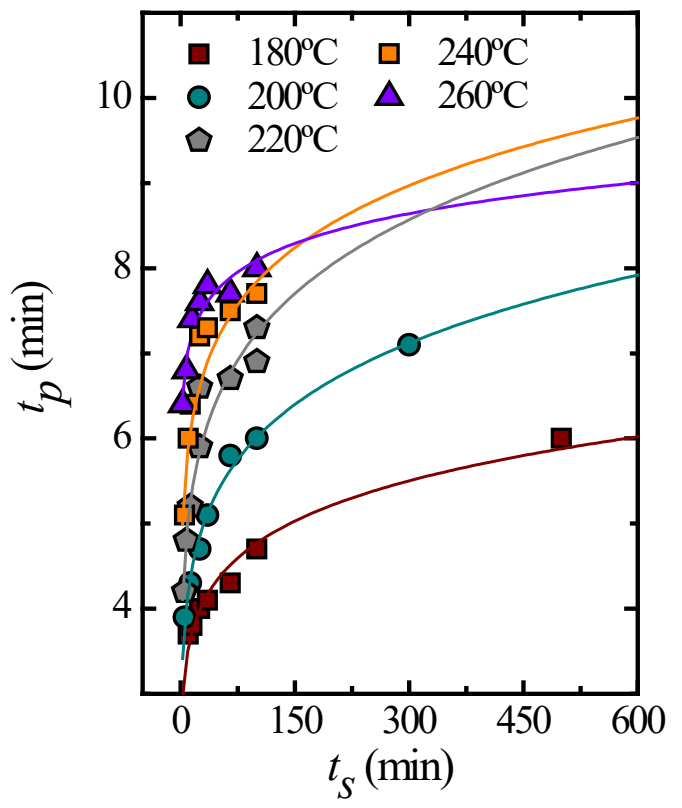


Figure 7. iPP crystallization peak-time $\left(t_{p}\right)$ as a function of holding time $\left(t_{s}\right)$ at the indicated $T_{s}$ temperatures $^{142}$.

Figure 7 shows the time needed to isothermally crystallize iPP (expressed as the peak crystallization time at constant $T_{c}$ values) as a function of holding time for different $T_{s}$ temperatures. Increasing the time spent at $T_{s}$ in the melt, the time needed to crystallize the sample increases, which indicates that the number of active nuclei is reduced. At temperatures that are well above the experimental melting temperature, i.e., 240 and $260{ }^{\circ} \mathrm{C}$, the crystallization rate after about $100 \mathrm{~min}$ is practically the same as the one after a holding time of $20 \mathrm{~min}$. Although the plateau value is not reached yet, the memory effect is clearly vanishing. Interestingly, this final crystallization time is independent of the adopted $T_{s}$ temperature, as long as $T_{s}$ is above the equilibrium melting temperature of iPP. At temperatures just above the experimentally measured melting temperature, the crystallization time does not reach the same plateau value, even when very long holding times (hours) are applied. Analyzing the kinetics of decay of the memory effect on crystallization at the different $T_{s}$, an activation energy of 89 $\mathrm{kJ} / \mathrm{mol}$ was calculated. Accordingly, Alfonso and Ziabicky ${ }^{142}$ proposed that the disappearance of clusters or athermal nuclei (self-nuclei) is controlled by chain selfdiffusion.

a)

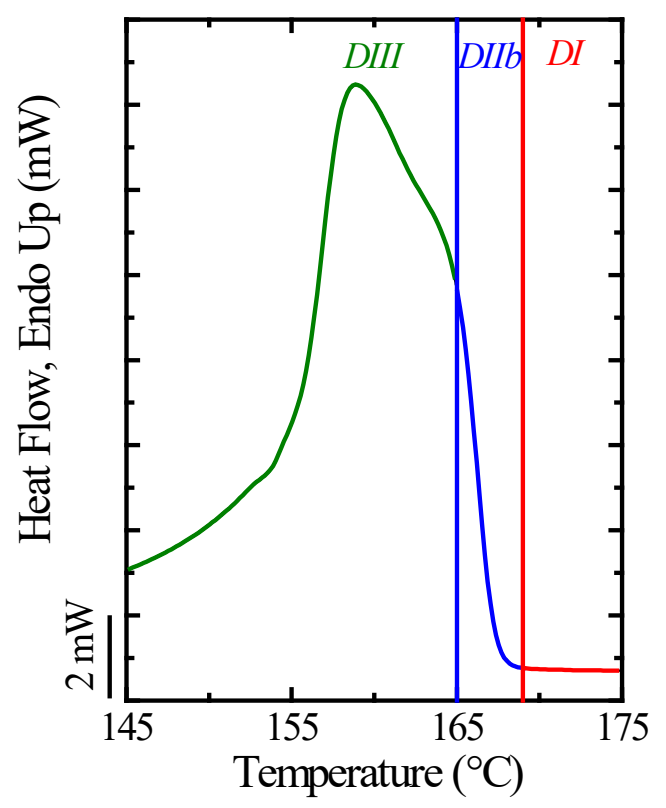

b)

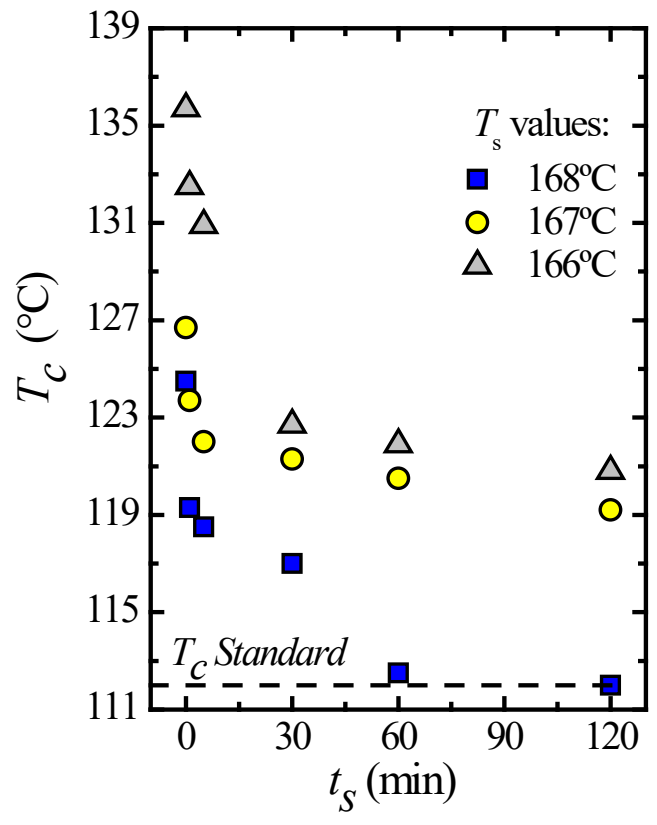


Figure 8. a) Standard melting endotherm of the iPP sample studied by Lorenzo et al. ${ }^{47}$. Vertical bars represent the limits between Domains. b) Dependence of the peak crystallization temperature $\left(T_{c}\right)$ of iPP as a function of the time spent at the different $T_{s}$ temperatures during self-nucleation in Domain II. Data taken from ref. 47.

The work of Lorenzo et al. ${ }^{47}$ should be highlighted since they studied the effect of the time spent at $T_{s}$ for temperatures within both Domain IIa and IIb in iPP and PCL. Figure $8 \mathrm{a}$ shows the melting DSC scan of the iPP sample employed by Lorenzo et al. ${ }^{47}$ together with the indication of the SN Domains. Figure $8 \mathrm{~b}$ shows the SN time dependence of crystallization temperature for the same polymer. Increasing the time spent at $T_{s}$, the nucleation density was reduced, and thus the crystallization temperature decreased. As could be expected, the decrease in the crystallization temperature depends on the values of $T_{s}$. At $T_{s}$ temperatures corresponding to Domain IIa (see for example, $T_{S}=168^{\circ} \mathrm{C}$ in Figure 8), the nucleation density was reduced within 1 hour to the characteristic values of the iPP sample whose melt memory effect has been erased (i.e., standard iPP sample in Domain I). On the other hand, at lower $T_{s}$ temperatures, within Domain $I I b$, even when maintaining the sample for 2 hours at $T_{s}$, the nucleation density remained higher than that in the standard iPP sample, implying that much longer times would be required. Analogous results were obtained with PCL samples of different molecular weights ${ }^{47}$.

Chen et al. ${ }^{143}$ have recently studied the effect of annealing time on the memory effect of ethylene/1-butene random copolymers by varying the time spent at the $T_{S}$ temperature. A hydrogenated polybutadiene (HPB) sample with a low molecular weight $\left(16,000 \mathrm{~g} / \mathrm{mol}\right.$ ) was heated to $140^{\circ} \mathrm{C}$ (a $T_{s}$ value corresponding to Domain IIa), which in this particular case is just above its equilibrium melting temperature $\left(T_{m}{ }^{0}=138^{\circ} \mathrm{C}\right)$, for times as long as $1200 \mathrm{~min}$. A reduction on the crystallization temperature of only $1^{\circ} \mathrm{C}$ was obtained, in comparison with holding the same sample for only $5 \mathrm{~min}$ at the same temperature. This indicates the very high stability of self-nuclei.

The authors ${ }^{143}$ have also analyzed the extent of melt memory, which they defined as the difference between the crystallization temperature of the sample cooled from an isotropic melt and that of the sample self-nucleated at $140^{\circ} \mathrm{C}$. The disappearance of the melt memory effect is more difficult with the increase of the molecular weight of the samples due to the increase in their melt viscosity, which 
results in an enhancement of the topological constraints that hinder the randomization of the polymer melt.

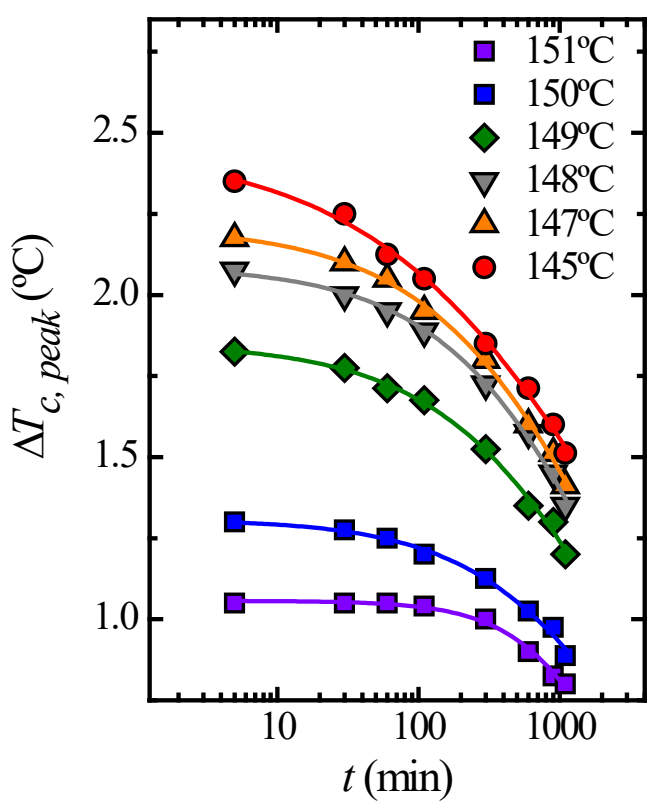

Figure 9. Strength of the melt memory effect as a function of annealing time at different selfnucleation temperatures for a random ethylene/1-alkene copolymer (denoted as P16 sample in their work) at different $T_{s}$ temperatures ${ }^{143}$.

In the case of a random ethylene/1-alkene copolymer, the extent of melt memory is reduced by increasing the $T_{s}$ temperature, however, it is not completely erased although annealing times as long as $1200 \mathrm{~min}$ are employed (Figure 9). In particular, the times for the dissapearance of melt memory effect are by far longer than the times involved in the reptation process of the polymer molecules. Chen et al. have been able to construct a master curve with the extent of melt memory effect versus annealing time, obtaining an activation energy from the "time-melt temperature" shift-factor of 1100 $\mathrm{kJ} / \mathrm{mol}$. Such value is much higher than, for instance, the values reported for the dissolution of flow-induced nuclei in iPP. The activation energy for the dissolution of nuclei is also larger than that of viscous flow, and this was explained by the fact that it is related with the detachment of chain stems from the nuclei and their subsequent diffusion, being the stem detachment the rate-limiting step of the process.

Summarizing, the literature reports different trends for the time dependence of memory effects. In some cases no effect of time was observed, even if times as long as several hours have been employed. However, if the temperature range of the different 
self-nucleation Domains is considered (in case of the works for which this data is available), it should be noted that the effect of holding time at $T_{s}$ becomes negligible for materials that are held at temperatures within Domain III. In the case of Domain IIb, where crystal fragments are still present, authors report either no effect or a reduction of nucleation density without reaching values similar to those of standard (non-selfnucleated) samples. Only few works report that upon increasing holding time a reduction of melt memory effect is obtained, until all self-nuclei disappear and the behavior reverts to that of a sample whose melt memory effect has been erased. This has been reported for materials that are in the upper temperature range of Domain IIa, therefore at temperatures close to the border with Domain I.

The persistence of the memory effect due to the presence of self-nuclei can be compared with that of flow-induced precursors. The kinetics of disappearance of the effect of flow on crystallization has been widely studied in the literature, on several polymers and with different techniques ${ }^{91,133,144,145}$. Generally, flow-induced effects on crystallization relax with characteristic kinetics which are much slower than rheological reptation times. The dissolution of these extra-nuclei is a thermally activated process, with activation energies ranging from 100 to $300 \mathrm{~kJ} / \mathrm{mol}$. Consequently, it is believed that the rate-determining step is not the relaxation of the oriented chain conformation, but rather the melting of quasi-ordered structures. Although some analogies between the dissolution processes of the two types of nuclei (self-nuclei and flow-induced nuclei) can be recognized, the differences in the disappearance temperatures and times are apparent when comparing the same polymer. For example, in iPP, flow-induced precursors can survive for few hundreds minutes ${ }^{91}$ at temperatures as high as $250{ }^{\circ} \mathrm{C}$, while usually melt memory disappear in a self-nucleated sample upon holding the polymer for a few minutes at $170{ }^{\circ} \mathrm{C}$. Therefore, the different nature of the two nonequilibrium melts can be clearly inferred.

It can be concluded that very long annealing times are needed to destroy selfnuclei or to erase melt memory effect in a self-nucleated sample in Domain II. The increase of annealing temperature (within Domain II) facilitates the erasure of melt memory by shortening the necessary holding time. Nevertheless, long times are still required (usually hours), and this probably explains why in some of the systems reported in the literature no effect of time is observed in the analyzed $T_{s}$ ranges and time intervals. The typically high activation energies associated to the disappearance of melt 
memory effects point towards a highly cooperative process involved in the dissolution of self-nuclei.

\subsection{Effect of molecular weight and chain topology}

Molecular weight also influences melt memory, since the number of entanglements per chain is a function of chain length. Blundell and Keller studied the effect of molecular weight on melt memory with PE single crystals obtained after dissolving PE in xylene and then crystallizing the material from solution ${ }^{39}$. They studied PE narrow molecular fractions with $M_{w}$ values of $6.8 \times 10^{3} \mathrm{~g} / \mathrm{mol}$ to $2.6 \times 10^{6} \mathrm{~g} / \mathrm{mol}$, with polydispersity ratios of 1.1 . To compare the results, they measured the $T_{\mathrm{s}}$ required to obtain crystals of a certain size, which corresponds to a predetermined constant number of nuclei. They found that to have the same crystal size with the different molecular weights, the $T_{s}$ required in each case was very different, from $95^{\circ} \mathrm{C}$ in the case of the lowest molecular weight fraction to $105^{\circ} \mathrm{C}$ in the case of the highest. These results imply that the melt memory effect increases as molecular weight increases. They speculated that nuclei are made by one single macromolecule, i.e., a high molecular weight chain from the end tail of the molecular weight distribution.

In subsequent work, Keller and Willmouth studied the sensitivity of SN technique to the high molecular weight fraction of the molecular weight distribution ${ }^{68}$. They found that the presence of a high molecular weight fraction increases the nucleation density and, by measuring the number of nuclei for purposely designed blends, they could correlate the formation of self-nuclei with the specific high-end tail of the molecular weight distribution.

More recently, several authors have studied the effect of molecular weight on the melt memory in bulk polymers. Lorenzo et al. ${ }^{47}$ and Pérez et al. ${ }^{56}$ studied PCLs of different molecular weights; they found that the transition temperature between Domains increased with the molecular weight. The width of Domain II, i.e., the temperature range at which self-seeding $(D I I b)$ and melt memory effects $(D I I a)$ are observed, also increased with molecular weight. In the case of PCL with a $M_{w}=10$ $\mathrm{kg} / \mathrm{mol}$, the width of Domain II was equal to $6^{\circ} \mathrm{C}$, whereas, for a sample with $65 \mathrm{~kg} / \mathrm{mol}$, Domain II had a larger width of $15^{\circ} \mathrm{C}$. ${ }^{47}$ This increase in melt memory effect can be explained considering that as molecular weight increases, the entanglements restrict the diffusion of the chains, which hinders the transition to an isotropic melt state. Thus, the 
sample has to be heated to higher temperatures to reach Domain I and a wider Domain II is obtained.

Similar results were reported for the case of ethylene/1-butene random copolymers (containing $2.2 \mathrm{~mol} \%$ of ethyl branches). Reid et al. ${ }^{52}$ observed that for samples with molecular weights lower than $4.5 \mathrm{~kg} / \mathrm{mol}$ the crystallization temperature is independent of $T_{s}$ temperature, or, in other words, there is no melt memory effect. For samples with higher molecular weights, the transition temperature between Domain II and Domain I, increases with molecular weight. Remarkably, this transition temperature becomes higher than the equilibrium melting temperature $T_{m}{ }^{0}$ for samples with molecular weights higher than $13.0 \mathrm{~kg} / \mathrm{mol}$, which corresponds to the critical molecular weight to obtain entanglements in polyethylene. The behavior of these random copolymers will be described in more detail in the next section.

In addition to the molecular weight, chain topology is also important for the melt memory effect. In Figure 10, the self-nucleation results obtained by Pérez et al. for PCLs having the same molar mass but composed of either linear or cyclic chains are shown $^{56}$. The width of Domain IIa, i.e., the temperature range at which memory effects occur, is wider for the linear sample than for the cyclic one. To explain the differences in Domain IIa widths, it should be noted that the entanglement density of linear and cyclic PCLs is different. In fact, in the case of cyclic PCL there are no chain ends, resulting in a lower entanglement density. Therefore, in the case of cyclic PCL lower temperatures are needed to reach an isotropic melt state or Domain I, which explains the narrower Domain IIa width in comparison with the linear PCL.

The results obtained on different polymers with varying chain lengths, as well as the comparison between linear and cyclic analogs at constant molar mass, clearly highlight the role of topological constraints, i.e., entanglements, in determining the ultimate stability of self-nuclei or disappearance of melt memory effect. Moreover, a minimum value of molecular weight is also required for the manifestation of the melt memory effect, as confirmed by the absence of self-nucleation in low molecular weight molecules. The melt memory effects on re-crystallization are thus strongly linked to the macromolecular nature of polymers. The experimental results summarized in this section agree with the recent molecular dynamics simulation work of Luo and Sommer ${ }^{55}$ that highlight the dependence of the memory effect on the relaxation of chain segments between entanglements (see also section 3 below). 
a)

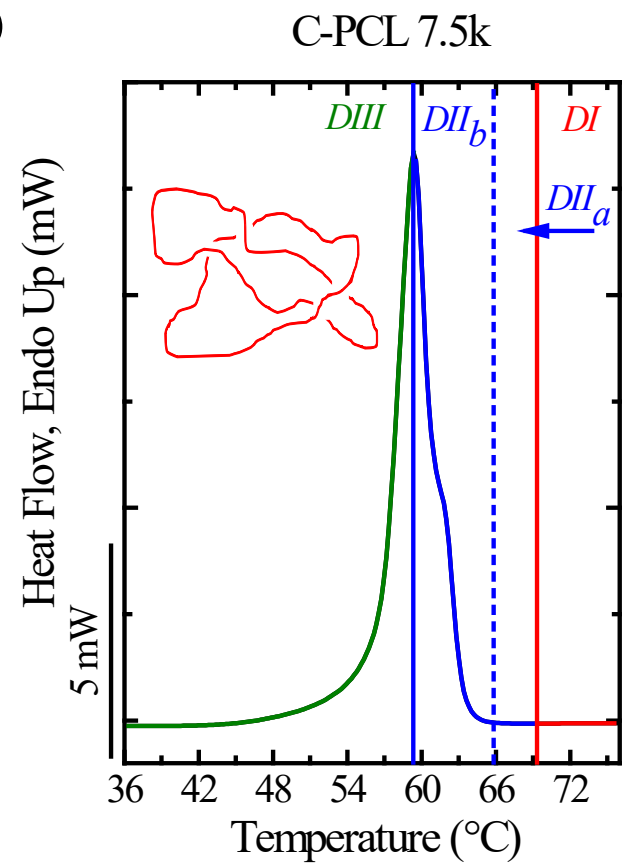

b)

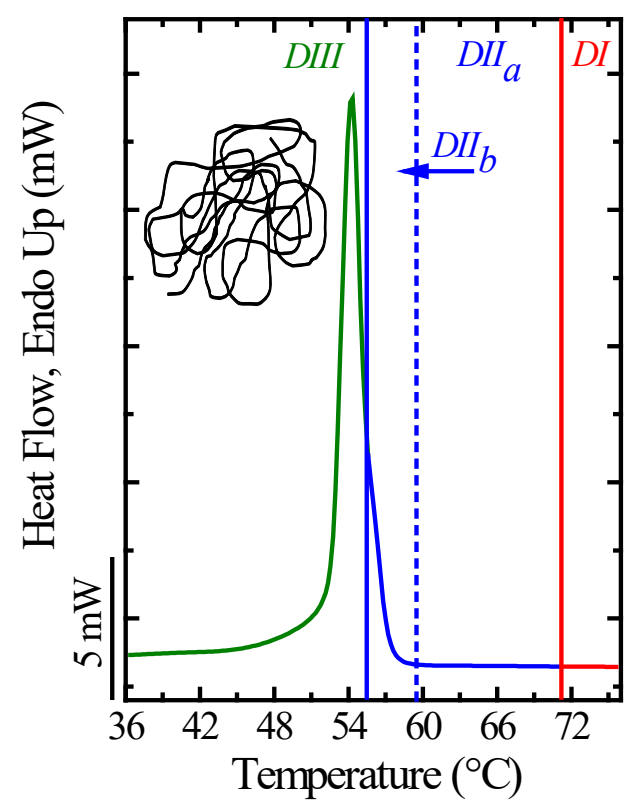

Figure 10. DSC heating traces showing the melting endotherms of cyclic and linear PCLs (with a number average molecular weight of $7.5 \mathrm{~kg} / \mathrm{mol}$ ), the different self-nucleation Domains are indicated by vertical lines ${ }^{56}$. Copyright 2014 Elsevier B.V.

\subsection{Self-nucleation in copolymers vs. homopolymers}

The self-nucleation behavior of different random copolymers has been analyzed in the literature. The groups of Alamo and $\mathrm{Hu}$ have analyzed ethylene/1-butene copolymers $^{52}$ with different content of branches. Figure 11 plots the crystallization temperature as a function of $T_{s}$ temperature for samples with different branch contents. 


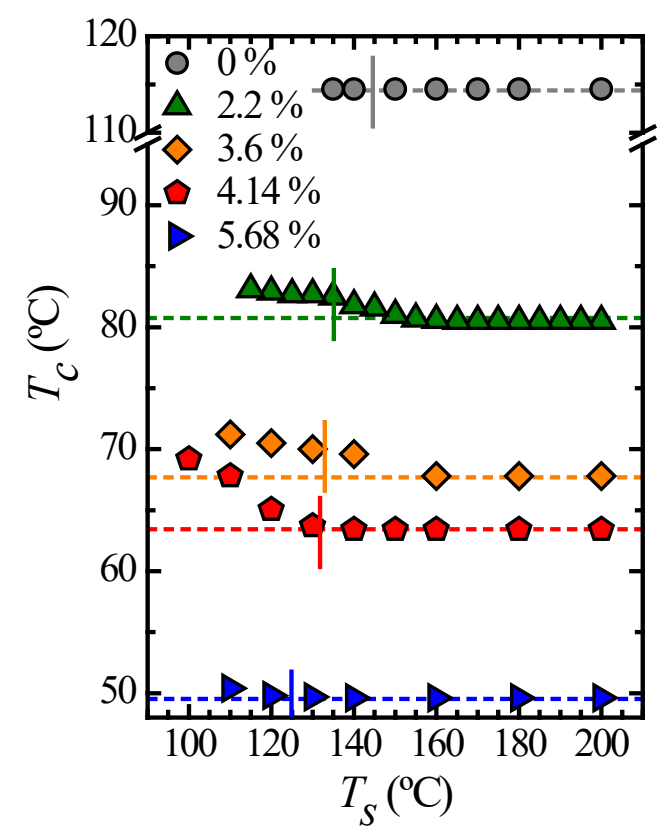

Figure 11. Crystallization temperature as a function of self-nucleation temperature for ethylene/1-alkene copolymers with different branching content ${ }^{52}$. The horizontal lines correspond to the standard crystallization temperature, whereas the vertical lines indicate the equilibrium melting temperatures of the different polymers. Data replotted from ref. 52.

Remarkably, they have found that these copolymers have a melt memory effect that persists at temperatures above the equilibrium melting point, whereas in the case of unbranched PE homopolymer (or for homopolymers in general) this behavior has never been observed. This result was attributed to the formation of a complex amorphous phase during crystallization, due to the partitioning of crystallizable sequences of proper length. During the crystallization process of random copolymers with non-crystallizable comonomers, chain sequences of adequate lengths have to diffuse from the melt to the crystal growth front, and in this way, a complex topology comprising knots and ties is formed in the melt. According to the authors, due to these topological constraints, temperatures higher than $T_{m}{ }^{0}$ are needed to obtain a completely isotropic melt. The width of the melt memory effect is reduced when the branch content increases. When the length of the crystallizable sequences is shortened, thereby reducing the crystalline fraction as well as the lamellar thickness, less topological constraints are created in the melt, and therefore lower temperatures are needed to obtain a completely isotropic melt state. 
Similar results were obtained by Mamum et al. for narrowly distributed ethylene/1-alkene random copolymers ${ }^{88}$. The highest temperature to observe memory effect increases with comonomer content, showing a maximum at $2 \mathrm{~mol} \%$, and then decreases. Again, this result was interpreted as the competition between the enhancement of topological constraints and the reduced crystallinity.

A different situation arises when studying copolymers with broad comonomer composition distribution. In this case, Mamun et al. ${ }^{88}$ observed a peculiar behavior. At high melting temperatures ( $T_{s}$ temperatures), the crystallization temperatures were constant, as an isotropic melt sate was obtained. Reducing the melt temperature $\left(T_{s}\right.$ temperature), an increase in $T_{c}$ was obtained, due to the presence of self-nuclei, but a further decrease of $T_{m}$ results in an unexpected reduction of $T_{c}$. This latter effect results from the liquid-liquid phase separation of molecular chains with different contents of comonomer. This liquid-liquid phase separation facilitates the diffusion of chain segments, effectively reducing the number of self-nuclei. For even lower $T_{m}$ temperatures, an increase in $T_{c}$ was obtained due to the presence of unmolten crystals (i.e., self-seeding). The occurrence of liquid-liquid phase separation was supported by small angle neutron scattering measurements ${ }^{146}$.

More recently, Marxsen and Alamo ${ }^{94}$ have compared precision and random polyethylenes with halogen substitution. Random copolymers of polyethylene with different content of $\mathrm{Br}$ and $\mathrm{Cl}$ atoms exhibited strong melt memory effects, even above the equilibrium melting point, showing similar behavior to that of ethylene/1-alkene copolymers. It has been observed that those random copolymers show memory effect until $6.67 \mathrm{~mol} \%$ of halogen substitution, and at higher halogen content, the memory effect disappears. The co-unit content for which the memory effect disappears is higher than in ethylene/1-alkene copolymers because some of the halogen atoms are included in the crystals.

However, in the case of precision halogen-substituted polyethylenes, the selfnucleation behavior is equivalent to that of PE homopolymers, as there is not a significant change in the crystallization rate when the sample is heated just above the melting point. Moreover, the authors have shown that recrystallization of those polymers depends on the original polymorph. The melt memory effect is very weak or disappears completely depending on the type of polymorph obtained.

The self-nucleation of a series of propene/ethylene random copolymers has been recently studied as a function of composition ${ }^{64}$. Self-nucleation experiments show that 
the DIIa width of the materials increases with the content of defects: for iPP that contains $1.17 \mathrm{~mol} \%$ of defects (regio- and stereo-errors) the width of Domain IIa is equal to $4^{\circ} \mathrm{C}$, whereas for $\mathrm{P} / \mathrm{E}$ with $10.1 \mathrm{~mol} \%$ of defects the width reaches a value of $30^{\circ} \mathrm{C}$. This large increase of the temperature range at which melt memory effect occurs can be explained considering the sequence partitioning proposed by Reid et al. in ethylene/1-alkene copolymers ${ }^{52}$ and explained above. However, contrary to the results observed for ethylene/1-alkene copolymers, the melt memory effect for all the propene/ethylene random copolymers samples disappeared at temperatures well below the equilibrium melting point. These differences in comparison with ethylene/1-alkene copolymers are ascribed to the lower melt viscosity of polypropylene with respect to that of PE, which enables faster diffusion of the chain segments and disappearance of the melt memory effect at temperatures below $T_{m}{ }^{0}$.

The studies of Alamo and $\mathrm{Hu}$ et al. ${ }^{52,88,94,110}$ highlight that partitioning of crystallizable sequences controls the memory effect of random copolymers. To sustain crystal growth, non-crystallizable comonomers need to diffuse away from the interface and accumulate in the amorphous regions, giving rise to a complex melt topology. As such, very high temperatures, even above the equilibrium melting point might be required to obtain an isotropic melt state and erase the memory effect. While this mechanism captures well the memory effect features observed in random copolymers, it is unable to explain the clear differences in self-nucleation behavior exhibited by various homopolymers. The possible different origins of the memory effects in the two polymer classes will be addressed in the subsequent section.

\subsection{Effect of confinement}

Since confinement has paramount importance in the crystallization process of polymeric materials, it is no surprise that it affects the self-nucleation behavior, as demonstrated for several confined systems, including block copolymer microdomains and nanocomposites.

In the case of block copolymers, the self-nucleation behavior depends on the degree of confinement, which in turn is related to the volumetric fraction of each phase and the segregation strength. Three different self-nucleation behaviors have been

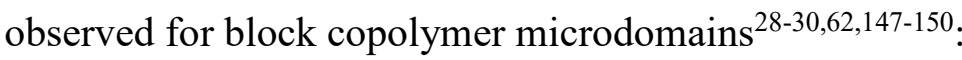

a. Classical self-nucleation behavior: The crystallizable block component shows the typical self-nucleation behavior characterized by three 
Domains $^{149-151}$. This behavior has been observed for crystallizable block components within: a) strongly segregated block copolymers when the block under consideration constitutes the matrix or percolated microdomains (such as lamellae or cylinders) and b) homogeneous or weakly segregated copolymers when the block under consideration crystallizes from a single-phase melt or provokes break-out during its crystallization.

b. $\quad$ Absence of the self-nucleation $\operatorname{Domain}^{148,149}$ (i.e., Domain II): The crystallizable block copolymer component is confined within microscopic isolated microdomains (typically cylinders or spheres) and shows only Domain I and Domain III. Domain II or self-nucleation Domain is absent due to the high number of microdomains (typically between $10^{12}-10^{16}$ microdomains $/ \mathrm{cm}^{3}$ ) that would require an equally high amount of self-nuclei. In order to generate such a high amount of self-nuclei, the temperature must be reduced to low values where the material is only partially molten. Therefore, these unmolten crystals undergo annealing, and the material reaches Domain III (i.e., the self-nucleation and annealing Domain).

c. Absence of self-nucleation Domain (Domain II) and partitioning of Domain III into two sub-Domains ${ }^{148,149,151}$ : in the case of block copolymers with a very high number of microdomains, the injection of self-nuclei into every microdomain is difficult. Thus, Domain III can be divided into two subDomains: $\mathrm{DIII}_{A}$ sub-Domain, in which annealing occurs in the absence of selfnucleation, and DIIISA sub-Domain, in which annealing and self-nucleation occur. This DIIISA sub-Domain is the equivalent to the standard DIII.

Arnal et al. ${ }^{151}$ have studied the self-nucleation behavior of PS- $b$-PEO- $b$-PCL copolymers, which contain two crystallizable blocks: PEO and PCL. First, the selfnucleation behavior of the PEO and PCL homopolymers was examined, observing that they show the classical self-nucleation behavior with three Domains. Then, they extended the study to PS- $b$-PEO diblock copolymer. When the PEO block forms the matrix (with PS block forming spheres or cylinders depending on composition) only one PEO crystallization peak is observed at the same supercooling as in PEO homopolymer. This PEO block shows classical self-nucleation behavior with the three Domains. However, the transition temperatures between DI/DII and DII/DIII are shifted to lower temperatures in comparison with PEO homopolymer; therefore the self- 
nucleation and annealing processes are somewhat hindered in the copolymer. On the other hand, when the composition of PEO is reduced in the copolymer to approximately $20 \%$, the PEO block forms isolated spheres in a PS block matrix. In this case, the PEO block crystallizes only at very large supercoolings, which indicates that homogeneous nucleation occurs. The high number of microdomains inhibits the self-nucleation process, in fact, with reducing $T_{s}$, crystal annealing occurs before self-nucleation, i.e., a direct transition from $D I$ to $D I I I_{A}$ is observed. At even lower temperatures $D I I I_{S A}$ is obtained, at which both self-nucleation and annealing occur. An analogous behavior to the PEO block was observed when the PCL block was self-nucleated.

The effect of nanoparticles' addition on the self-nucleation behavior has been investigated in the literature. Trujillo et al. ${ }^{30,152}$ have studied HDPE/carbon nanotubes (CNT) nanocomposites prepared by in situ polymerization and containing different types of CNTs: single wall (8-32\%) and double wall (\% 8) carbon nanotubes. The HDPE homopolymer employed showed only Domain I and Domain III. The absence of self-nucleation Domain or Domain II is attributed to the high number of active heterogeneities present in this material, as in many HDPEs. The presence of an extremely high heterogeneous nucleation density does not allow further increases in the total concentration of nuclei with self-nuclei.

Similarly, in the case of nanocomposites, Domain II is absent. However, the transition temperature from Domain I to Domain III is higher for nanocomposites in comparison with neat HDPE. It should be noted that for these samples, nucleation efficiency values between 199 and $298 \%$ are obtained (these efficiencies are calculated employing $T_{c}$ values obtained after $\mathrm{SN}$ in the upper temperature limit of Domain III as a reference because Domain II is absent). The nucleation efficiency of CNTs was found to be higher than HDPE's own self-nuclei, an effect termed supernucleation and, in this particular case, related to the growth of the chains on CNT surface.

Colonna et al. $^{153}$ prepared poly(butylene terephthalate) containing reduced graphene oxide (rGO). These nanocomposites were prepared by in-situ polymerization. Neat poly(butylene terephthalate) shows the three classical self-nucleation Domains: at temperatures higher than $230^{\circ} \mathrm{C}$, Domain I is observed, at temperatures between 230 and $227^{\circ} \mathrm{C}$, Domain II is encountered, and finally, at temperatures equal or below $226^{\circ} \mathrm{C}$, Domain III is achieved. To quantify the nucleation efficiency of rGO, self-nucleation studies of the nanocomposites were performed. From these experiments, it was found that when $10 \%$ of rGO is added only $D I$ and $D I I I$ are found, while the self-nucleation 
Domain II disappears. Employing the equation proposed by Fillon et al. ${ }^{100}$, a nucleation efficiency of $270 \%$ is obtained for one of the nanocomposites containing rGO. In this particular case, the supernucleation effect is associated with the presence of extended chain crystals on rGO layers.

More recently, Wen et al. ${ }^{154}$ have studied the self-nucleation behavior of methoxypolyethylene glycol (MPEG) grafted onto nanosilica. They have studied MPEG of different molecular weights (2000 and $4000 \mathrm{~g} / \mathrm{mol})$ and the effect of different grafting densities. The nanocomposites prepared by Wen et al. have a very high content of nanosilica, between 68 and $80 \%$ by weight. Reducing the grafting density, there is a higher amount of silica in the nanocomposites, thus increasing the confinement experienced by the polymer. In the case of neat MPEG of $2000 \mathrm{~g} / \mathrm{mol}$, three selfnucleation Domains are observed, being the width of Domain $I I$ about $3^{\circ} \mathrm{C}$ and mostly on top of the melting peak (i.e., crystal fragments are present, DIIb). For nanocomposites, at low grafting densities, only $D I$ and $D I I I$ are observed due to the confined crystallization of the material. However, with increasing the grafting density the degree of confinement is reduced because the nanoparticle content is decreased, so Domain II re-appears; with a temperature extension wider than in neat MPEG and equal to $5^{\circ} \mathrm{C}$, showing both DIIa and DIIb. A similar trend is observed with MPEG of 4000 $\mathrm{g} / \mathrm{mol}$.

In essence, confinement of macromolecular chains in isolated block copolymer microdomains or by tethering to the surfaces of nanoparticles profoundly affects the self-nucleation behavior. In all cases, strong confinement leads to the disappearance of Domain II. In some specific cases, where weak confinement is present, an extension of the temperature window of Domain II could be found. These results suggest that the relevant length scale for the self-nucleation phenomenon is indeed just a few tens of nanometers, commensurate to the lamellar thickness dimensions. It is worth noting that although for many aspects we consider self-nucleation as a peculiar case of homogeneous nucleation, this latter nucleation modality is not hampered by confinement in nanodomains.

\section{Interpretation(s) of melt memory effects}

The large number of experimental observations, part of which has been described in the previous section, has led the scientific community to propose several 
interpretations of melt memory effects. To a first approximation, the various views mainly dispute the very nature of self-nuclei, and in particular, on considering them either truly crystalline remnants or non-crystalline entities. We feel that this dichotomy has been somehow over-emphasized in the literature. In fact, as we briefly anticipated in Section 1, both situations of crystalline memory (self-seeding) or melt memory (selfnucleation) might exist, depending on the particular experimental conditions or polymeric systems under study. In the following, we will briefly illustrate the proposed interpretations in this wider framework.

The origin of crystalline memory effects was attributed to the presence of "annealed (stabilized) crystal fragments" by Fillon et al. ${ }^{43}$ in their seminal work on the calorimetric investigation of iPP self-nucleation. It should be noted that the employed self-nucleation temperatures corresponded to the high-temperature tail of the melting endotherm, in fact, Domain IIa, or melt memory Domain, in the employed iPP sample, was very small or nonexisting ${ }^{43}$.

Reiter et al. arrived at a similar conclusion to that of Fillon et al. $^{43}$ by investigating the recrystallized morphologies of polymer single crystals in thin films ${ }^{49,53}$. Via a self-seeding procedure, the authors generated in the second crystallization step arrays of small crystallites of uniform size and shape, which inherited the molecular orientation from the initial single crystal. The concentration of these "clones" crystals decreases strongly with increasing seeding temperature (Figure 12a), but the phenomenon was found independent of the duration of the thermal treatment at that temperature, for times up to one hour. Both the lack of a kinetic control in the self-seeding process and the correlation between the orientation of the unit cell vectors of re-grown crystals and the initial single crystal, led the authors to the conclusion that sub-microscopic crystalline remains could resist the melting procedure, due to a spontaneous lamellar thickening and enhanced stability.

It should be noted that, while the above arguments certainly support the proposed interpretation, they might not be conclusive. In fact, the explored range of melting time is relatively short, in comparison to the typical surviving time exhibited by melt memory effects (see section 2.2). 
a)

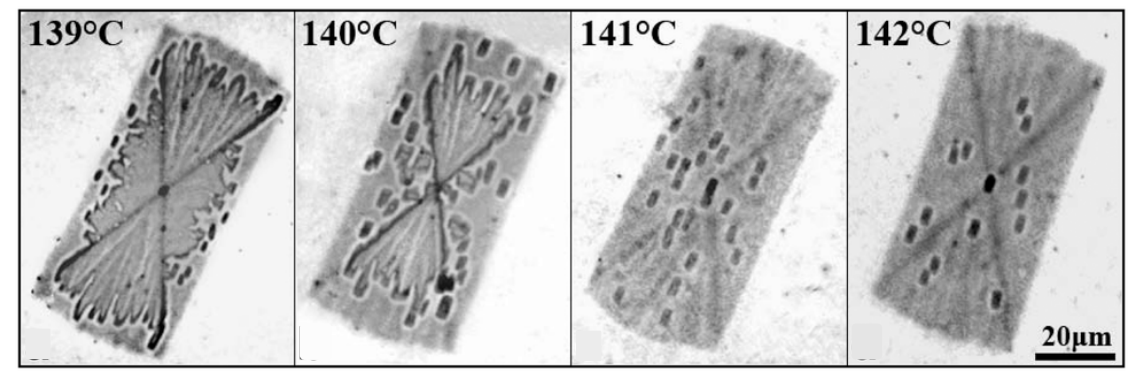

b) i)

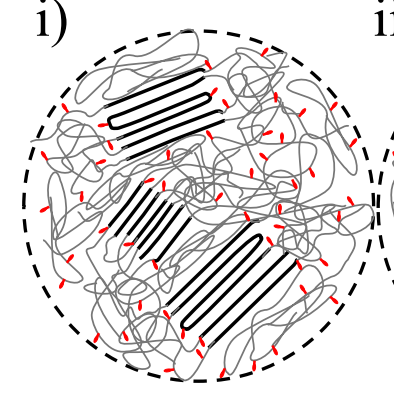

Copolymer ii)

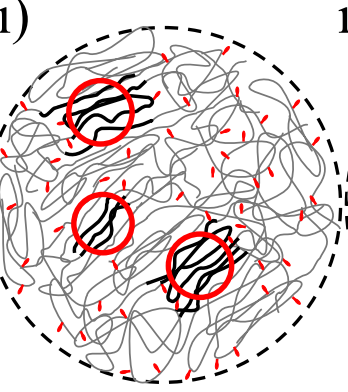

Heterogeneous melt iii)

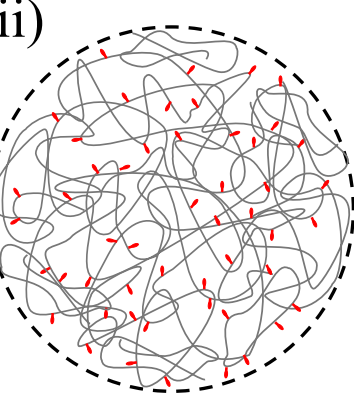

Homogeneous melt Semi-crystalline structure

c)

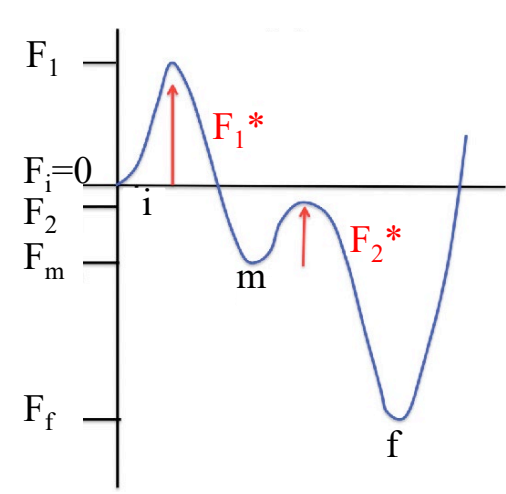

d)

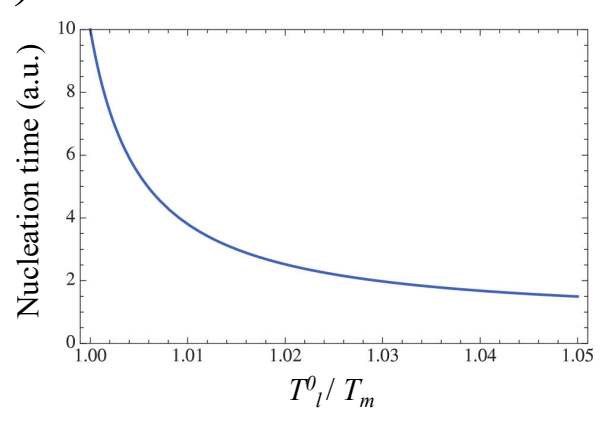

Figure 12. a) Optical micrographs showing "re-grown" poly(ferrocenyl dimethyl silane) crystals after self-nucleation for a short time at the indicated temperatures from ref. 53. Copyright 2014 Royal Society of Chemistry; b) Scheme illustrating melt memory effects in random copolymers. From left to right: i) semi-crystalline structure, after selection of the longest crystallizable sequences and partitioning of the comonomer into the amorphous phase, ii) heterogeneous (non-isotropic) melt with clusters of defect-free chain segments surrounded by topological constraints, iii) homogeneous melt with random distribution of crystallizable sequences and comonomers from ref. 52; c) Free energy variation for the crystallization process occurring either from an isotropic melt ("i") or metastable heterogeneous melt state ("m"). The crystalline energy minimum is indicated with " $\mathrm{f}$ ". The reaction coordinate is the overall crystallinity. Reproduced with permission from ref. 60. Copyright 2016 AIP Publishing, LLC.; d) Example of the dependence of nucleation times on the ratio between isotropization temperature of the heterogeneous melt and self-nucleation temperature before crystallization (denoted as $T_{m}$ in ref. 60). See ref. 60 for details. Copyright 2016 AIP Publishing, LLC. 
For what concerns the inheritance of crystalline orientation between the different "crystal generations", it is interesting to underline that the same effect could be found in molecular dynamics simulation experiments on single crystalline seeds by Luo and Sommer ${ }^{55}$. However, in this case, the complete melting of the original morphology was assured, and the behavior was attributed to the slow relaxation of the local entanglement topology. In addition, other experimental results showed that when longer holding times at the self-nucleation temperature are used, the re-grown crystals become more and more randomly oriented ${ }^{53}$.

Despite the above comments, the existence of un-molten crystalline fragments could certainly be the origin of melt memory effects, as suggested by Fillon ${ }^{43}$ and Reiter $^{49,53}$, under certain specific conditions. In particular, this situation is most likely to contribute to self-nucleation in some homopolymers, at temperatures superposed or slightly above the end of the melting endotherms (i.e., the temperature range within Domain IIb). In cases like PBS (Figure 5) and PCL (Figure 10), where Domain IIa extends well above the end of the experimentally recorded melting endotherm (up to 15 ${ }^{\circ} \mathrm{C}$ above it), it is unlikely that any crystal seeds remain, as described in section 2. However, from a strict thermodynamic point of view, the possible persistence of "undetectable "residual crystals cannot be excluded at self-nucleation temperatures below the equilibrium melting point. Although most of the literature typically refers to the easily experimentally accessible nominal melting temperature, rather than to the equilibrium value, for most of the homopolymers melt memory effect seldom exceeds $T_{m}{ }^{0}$.

The presence of self-seeds (crystal remnants) can be safely ruled out as a possible cause of melt memory in the case of random copolymers (see section 2.4). In fact, Alamo and $\mathrm{Hu}$ et al. reported the observation of enhanced re-crystallization kinetics in random ethylene/1-butene and ethylene/vinyl halide copolymers for selfnucleation temperatures ranging from about 5 to $25^{\circ} \mathrm{C}$ above the respective equilibrium melting temperature ${ }^{52,94}$. Obviously, such effects cannot arise from any crystalline remnants. Similarly, random propene/ethylene copolymers with ethylene content up to $10 \mathrm{~mol} \%$ show self-nucleation at temperatures up to $30{ }^{\circ} \mathrm{C}$ above the nominal melting temperature. Although in this case, the Domain I/Domain II transition temperature is lower than the equilibrium melting temperature, the survival of any crystal at this overheating is hard to justify, also in view of the hindered thickening process of the 
lamellae expected for these copolymers, due to the limited crystallizable sequence lengths along the chain.

Thus, Alamo and $\mathrm{Hu}$ et al. ${ }^{52,110}$ attributed the melt memory effect found in random copolymers to clusters of relatively long ethylene sequences, with negligible structural order, which remain in close proximity upon crystal melting (Figure 12b). The selection of regular sequences causes this weak segregation or melt heterogeneity during crystal growth, and its disappearance to give a randomized and homogeneous melt can only occur very slowly, due to the constrained amorphous phase topology (containing loops, ties and entanglements) ${ }^{52,110}$. This view is substantiated by the effects of time and molecular weight on the crystallization kinetics enhancement, as described in sections 2.2 and 2.3. Accordingly, no meaningful memory effects were found by the authors upon self-nucleation of polyethylene homopolymers. We underline that the drawing in Figure $12 \mathrm{~b}$ should be considered as a schematic representation only. In particular, there is no actual need for the defect-free chain in the clusters to adopt a strict parallel alignment, as more recently pointed out by Men et al. ${ }^{138}$ This is especially true under crystallization conditions for which the critical size of a primary nucleus is smaller than the size of such segregated region, as in that case crystallization of the longest crystallizable sequences can effectively occur as in an homopolymer melt. ${ }^{138}$

As previously mentioned, a Domain II extending considerably above the end of the melting endotherm is also found in homopolymers as well, such as in the aliphatic polyesters poly( $\varepsilon$-caprolactone) and poly(butylene succinate $)^{47,57}$. Despite such behavior cannot be explained with the concepts outlined above, valid for random copolymers which undergo defects partitioning upon crystallization, the role of a "frozen topology" of polymer melts can still be valid. Indeed, Luo and Sommer ${ }^{55}$ showed by means of molecular dynamics simulations and primitive path analysis of poly(vinyl alcohol) chains, that melt memory effects are related to the persistence of partially disentangled regions after melting, where nucleation upon supercooling the melt occurs preferentially.

Next to the suggestions that persistent annealed crystal fragments (Figure 12a) or non-isotropic melts (i.e., heterogeneous melt in Figure 12b) induce self-nucleation, the existence of residual non-crystalline order above the nominal melting temperature has also been widely discussed in the literature. Several hypotheses on the exact nature of these ordered "aggregates", surviving crystal melting in self-nucleated polymers, have been put forward. 
Strobl and coworkers ${ }^{82}$ rationalized crystalline memory effects by postulating that a crystallization precursor phase is formed first. As crystallization proceeds, the precursors domain volume fills with crystals and grows with crystallization time. During melting, this precursor, which they denote as a "conceptual object", is progressively emptied. Therefore, Strobl et al. consider that melt memory effects are related to the creation rate of the crystals within the precursor phase, which is a function of melting temperature. Accordingly, the development of a mesomorphic phase with liquid-crystal like properties has been suggested to precede crystal formation in poly(ethylene-co-octene) melt below Domain I/Domain II transition temperature ${ }^{82}$. However, its presence could only be indirectly inferred by changes in the crystallizing morphology and its mechanism, while no evidence of dissimilar melt structures could be gathered, for the admittedly very low difference in density between isotropic and liquid crystalline state.

On the other hand, experimental evidence exists for several systems, indicating that short-chain segments with ordered conformations, analogous to the one in the crystal, can exist up to temperatures considerably higher than the nominal melting point, where all the long-range crystalline order is destroyed ${ }^{106,155,156}$. Notably, a close link between persisting ordered conformations in the melt and memory effects on recrystallization has been established. For example, short helical and trans-planar sequences of syndiotactic polypropylene dissolve completely to an isotropic melt at temperatures related to the disappearance of melt memory effects ${ }^{156}$. In isotactic polystyrene, the concentration of chain segments comprising 5 monomers arranged in $3{ }_{1}$-helical conformation, which survive at a given melt temperature, shows a remarkable correlation with the maximum number of nuclei obtained upon re-crystallization from that $T_{s}{ }^{106}$.

A different kind of non-crystalline order which might be present in the melt has been proposed by Lorenzo et al. ${ }^{47}$, in order to explain the wide Domain II evidenced in PCL. The authors hypothesized that upon melting, although strict crystalline register between neighboring chain segments is lost, some residual (parallel) segmental orientation can be preserved. Such small bundles of aligned chains act as self-nuclei upon re-crystallization. More recently, a low concentration of strongly aligned polymer chains, obtained by coalesced inclusion compounds of various polymers with cyclodextrins molecules, has been shown to efficiently self-nucleate an isotropic melt of the same polymer ${ }^{157,158}$. These experiments corroborate the interpretation of Lorenzo et 
al. ${ }^{47}$, although the role of partial disentangled melt topology cannot be ruled out. In the next section, we will show some convincing evidence that residual interactions between chain segments can constitute a kind of non-crystalline melt structure that strongly influences memory effects.

Ziabicki and Alfonso ${ }^{131,159}$ proposed the first theoretical approach to melt memory effects in polymer crystallization. Based on classical nucleation theory, the authors ascribed the role of thermal history on crystallization kinetics to the persistence of non-equilibrium distribution of clusters size. In fact, above the melting temperature, an equilibrium concentration of molecular clusters of various sizes exist in the melt, with the shape of the concentration vs. size distribution being dictated by the free energy barrier for nucleation. When the polymer is supercooled, the clusters with sizes above the temperature-dependent critical dimension becomes "kinetically stable" nuclei. Memory effects can be observed because of the gradual disappearance of large clusters that are left from previous structures. The higher-than-equilibrium concentration of large clusters after incomplete relaxation of the cluster size distribution function directly results in a higher number of predetermined nuclei upon re-crystallization, rather than in faster nucleation rate. The proposed theory has been successfully applied for explaining the decreasing crystallization rate of iPP with increasing melting temperature and time in a related experimental work ${ }^{142}$.

More recently, Muthukumar developed a different theory describing the nucleation rate for a polymer with melt memory, assuming the existence of an intermediate (metastable) heterogeneous melt state in the pathway between the isotropic melt and the crystalline phase ${ }^{60}$. Such non-isotropic melt is characterized by an equilibrium isotropization temperature $\left(T^{\circ}{ }^{\circ}\right)$, which can be either below or above the equilibrium melting temperature of the crystalline state. Therefore, the developed theory can capture all different kinds of observed memory effects.

The generic free energy landscape below the equilibrium melting temperature is sketched in Figure 12c. The initial (isotropic) melt state, the heterogeneous metastable state, and the final crystalline state are indicated with the letters $i, m$, and $f$, respectively. The various states are characterized by the corresponding free energies $\left(F_{i}, F_{m}\right.$ and $\left.F_{f}\right)$, and separated by the energy barriers $F_{1} *$ and $F_{2}{ }^{*}$. Given that after equilibration of the system at $T_{m}$ both melt states have a finite probability of existence, crystallization can occur simultaneously by two different pathways: either from the isotropic state, by crossing the two energy barriers successively, or from the intermediate heterogeneous 
state, by surpassing the barrier between $m$ and $f$ only. Accordingly, the nucleation rate at a certain crystallization temperature can be expressed as a function of $F_{1}{ }^{*}, F_{2}{ }^{*}$, the selfnucleation temperature (which corresponds to $T_{m}$ in this particular case) and the equilibrium isotropization temperature of the heterogeneous melt state. An example of the calculated nucleation times for different ratios of $T_{i}{ }^{\circ}$ to $T_{m}$ is shown in Figure $12 \mathrm{~d}$. The nucleation rate decreases as the self-nucleation temperature approaches the limit for isotropization of the metastable melt state.

Muthukumar's theory certainly captures the major features of melt memory effects on re-crystallization kinetics qualitatively, however, quantitative experimental verifications are still lacking. This is mainly due to the inherent difficulties in measuring nucleation kinetics in many self-nucleated polymers. Nevertheless, a decrease of free energy barrier for primary nucleation with decreasing self-nucleation temperature has been measured for isotactic polystyrene ${ }^{106}$. On the other hand, Lorenzo and Müller measured the isothermal overall crystallization kinetics with DSC for different polymers cooled either from Domain I or from the optimal self-nucleation temperature ${ }^{160}$. They found that the undercooling dependence of the overall crystallization kinetics of the self-nucleated polymers (as determined by DSC) could be perfectly described by the Lauritzen and Hoffman growth barrier only, implying a control of the crystal growth step on the overall crystallization process, with no apparent barrier for primary nucleation. However, the data are not necessarily in conflict with the theory described above, since they might simply indicate that the free energy barrier for nucleation with melt memory is negligible in comparison with the barrier for crystal growth (secondary nucleation).

\section{Recent experiments with combined techniques: the role of intermolecular}

\section{interactions between chain segments}

As outlined before, several hypotheses about the nature of self-nuclei have been put forward. The lack of consensus, or at least of a more precise description of selfnuclei structure arises from the difficulties to detect them using conventional techniques that are sensitive to crystalline order, such as X-ray scattering ${ }^{52}$ or $\mathrm{AFM}^{49}$. In fact, the presence of self-nuclei or melt memory effects is typically indirectly detected by $\operatorname{DSC}^{43}$, FTIR ${ }^{61,63}$ or PLOM $^{57}$, by measuring the crystallization temperature, induction time for crystallization or the nucleation density after a given melt treatment. 
DSC experiments are the most adequate to determine the temperature ranges corresponding to each self-nucleation Domain and to ascertain the width of Domain II. However, for gaining insights about the detailed nature of self-nuclei, techniques that can measure directly intrinsic properties of the melt are needed. Employing NMR techniques, several researchers ${ }^{48,161-163}$ have tried to analyze the relaxation time of isotropic and self-nucleated melts, but the results obtained are not conclusive. However, more recently, it has been found that rheology and dielectric relaxation techniques can detect meaningful differences between self-nucleated and isotropic melts ${ }^{64,65,97}$.

a)

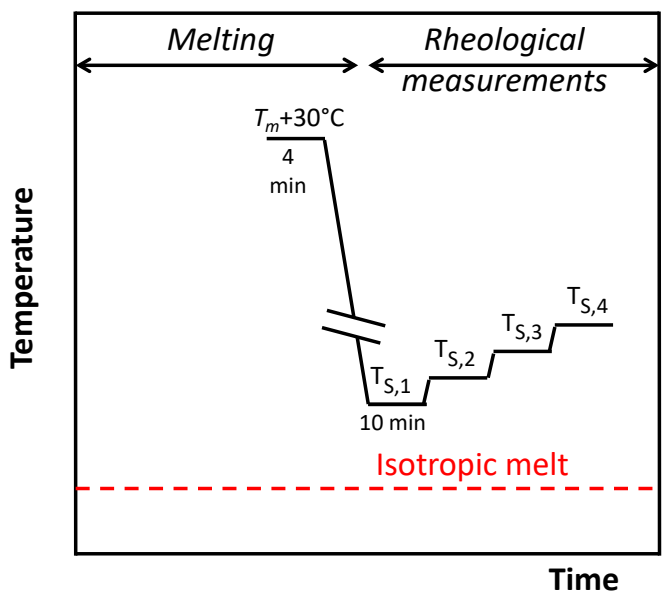

b) Self-nucleated melt

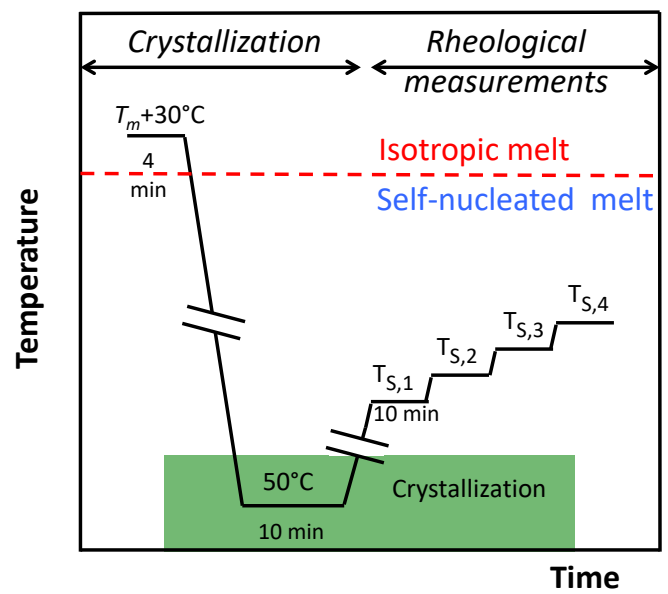

Figure 13. Scheme of the thermal protocols employed to study the rheological properties of: a) isotropic melt state and b) self-nucleated melt state. The difference between the two thermal procedures lies in the initial stages. In the first case (a), the sample is cooled down directly from the isotropic melt to the measurement temperature without any crystallization occurring during cooling. In the case of the selfnucleated melt, the sample is first crystallized by cooling and then heated to measurement temperature in a melt whose thermal history has not been erased (a self-nucleated melt).

Few polymers have been studied by these techniques, employing designed thermal protocols to be able to probe either isotropic or self-nucleated melt states at the same temperatures ${ }^{64,65,97}$. The applied thermal histories are summarized in Figure 13. To analyze the properties of an isotropic melt, the sample is first completely molten above the temperature for Domain I/Domain II transition (TDI/DII), and then cooled down until the measurement temperatures $\left(T_{s, 1}\right.$ etc.). Crystallization does not occur in the measurement time due to the extremely slow nucleation and crystallization kinetics at such low supercoolings in the absence of self-nuclei (Figure 13a). On other hand, to study the self-nucleated melt, the sample is also molten above TDI/DII, cooled down to 
obtain a standard crystalline state and then heated again until a measurement temperature in the molten (but self-nucleated) state ( $T_{s, l}$ etc.), below TDI/TDII (Figure 13b). By applying these thermal procedures the rheological or dielectric measurements can be performed at the very same temperature, but in two different melt states (isotropic vs. self-nucleated).

Sangroniz et al. studied melt memory effects in random propene/ethylene $(\mathrm{P} / \mathrm{E})$ copolymers by rheological methods ${ }^{64}$. Copolymer samples with different amounts of ethylene were employed. Increasing the ethylene content within the random copolymer induced a stronger melt memory effect, as the width of Domain II (or the temperature range at which melt memory effect occurs) increased.

No differences between isotropic and self-nucleated melt were observed regarding basic rheological parameters, such as melt viscosity, elastic and loss moduli and relaxation time for the studied $\mathrm{P} / \mathrm{E}$ copolymer samples. However, the results indicated that isotropic and self-nucleated melts display different thermo-rheological behavior. The isotropic melt state of propene/ethylene copolymers exhibits a thermorheologically simple behavior or, in other words, the application of the timetemperature-superposition (TTS) principle was possible and verified, as indicated by the overlay of the phase angle vs. complex modulus curves obtained at different temperatures (Figure 14a). On the other hand, the self-nucleated melt state displays a thermo-rheologically complex behavior, which was indicative of the presence of melt heterogeneities (Figure 14b).

These heterogeneities are attributed to persisting intermolecular interactions between chain segments (due to Van Der Waals forces) that were previously present in the crystal. The irreversible loosening of these interactions with increasing temperature brings about the thermo-rheological complexity of the self-nucleated melt. 
a)

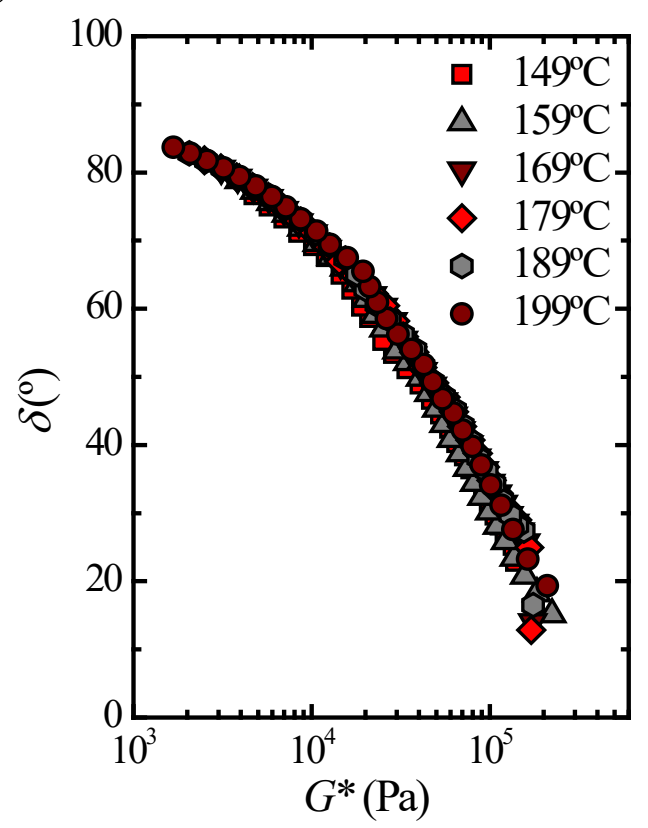

c)

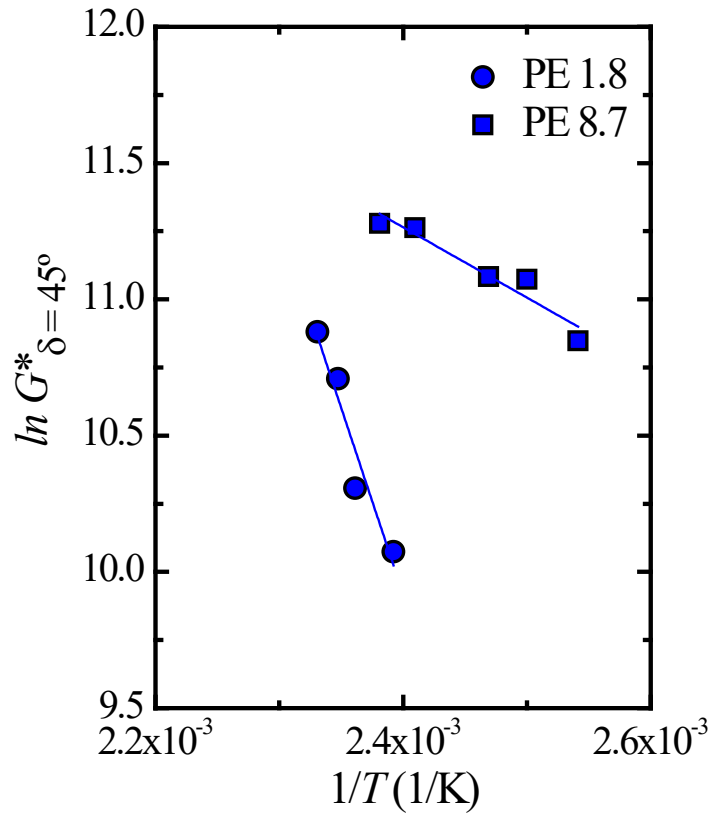

b)

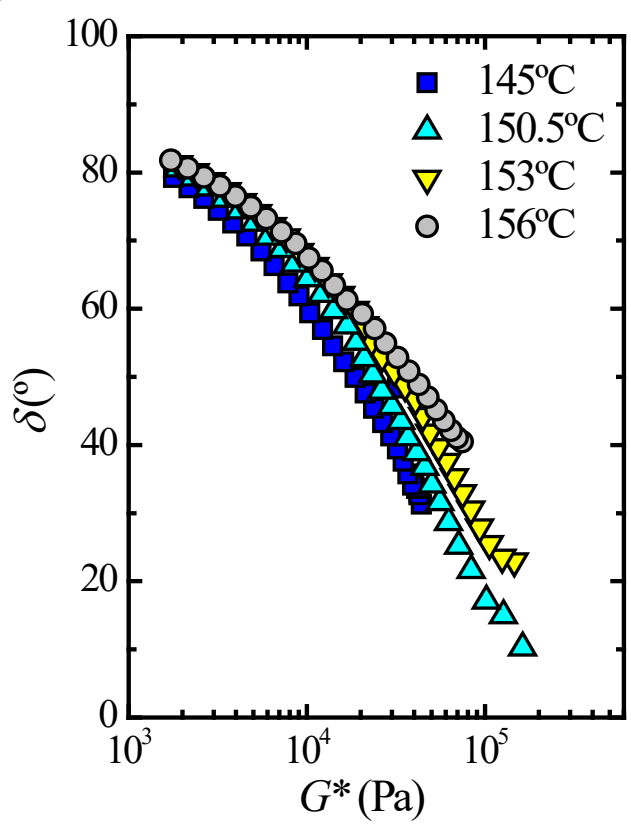

d)

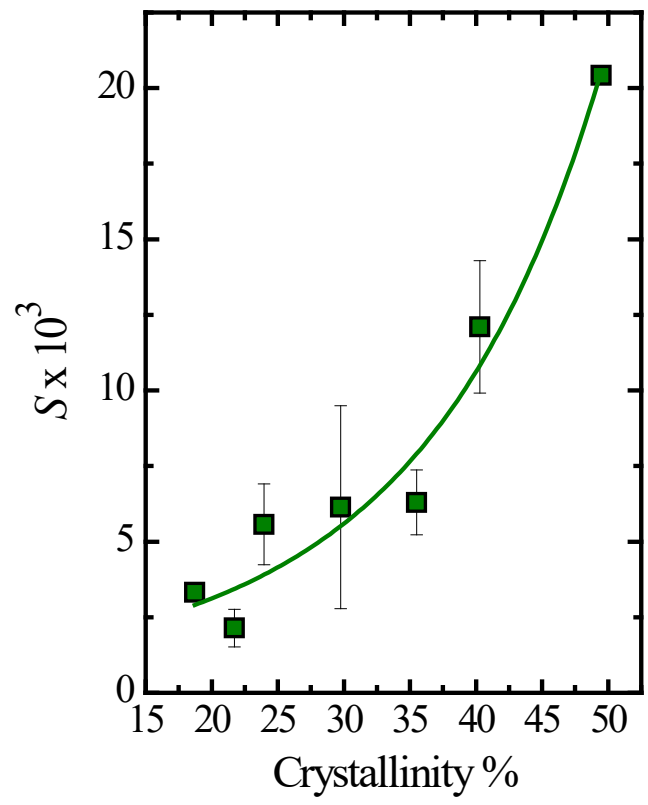

Figure 14. Phase angle versus complex modulus at different temperatures for propene/ethylene copolymer with $1.8 \%$ of defects for the isotropic melt a) and self-nucleated melt b); c) The natural logarithm of complex modulus at phase angle $=45^{\circ}$ as a function of the inverse of temperature for propene/ethylene copolymer containing 1.8 and $8.7 \%$ of defects. The slope of the curves is defined as $S$ and reflects the thermo-rheological complexity degree; d) $S$ parameter as a function of crystallinity for a series of propene/ethylene copolymers, the line corresponds to an arbitrary exponential fitting. Adapted from ref 64 . 
The degree of thermo-rheological complexity was empirically quantified, by evaluating the natural logarithm of the complex modulus measured at a phase angle of $45^{\circ}$, with respect to the reciprocal measurement temperature (Figure 14c). The steepness of the slope in this type of plot indicates the degree of thermo-rheological complexity of the melt. Considering the different copolymers, which are characterized by a different crystallizability, a remarkable correlation between the degree of crystallinity and the thermo-rheological complexity was found (Figure 14d). This strengthens the conclusion that the intermolecular interactions causing deviation from the TTS behavior in the $\mathrm{PP} / \mathrm{PE}$ self-nucleated melt are originated from the pre-existing crystals.

As intermolecular interactions seem to play a relevant role in the melt memory effect, Sangroniz et al. extended their studies to PCL, a polar polymer for which dipoledipole interactions are present in addition to the weak Van Der Waals forces ${ }^{65,97}$. The rheological behavior of the different melts was explored in a wide frequency range, encompassing both the terminal and rubbery zones (low and high frequency, respectively), from which the Newtonian viscosity and entanglement plateau modulus could be measured ${ }^{97}$.

a)

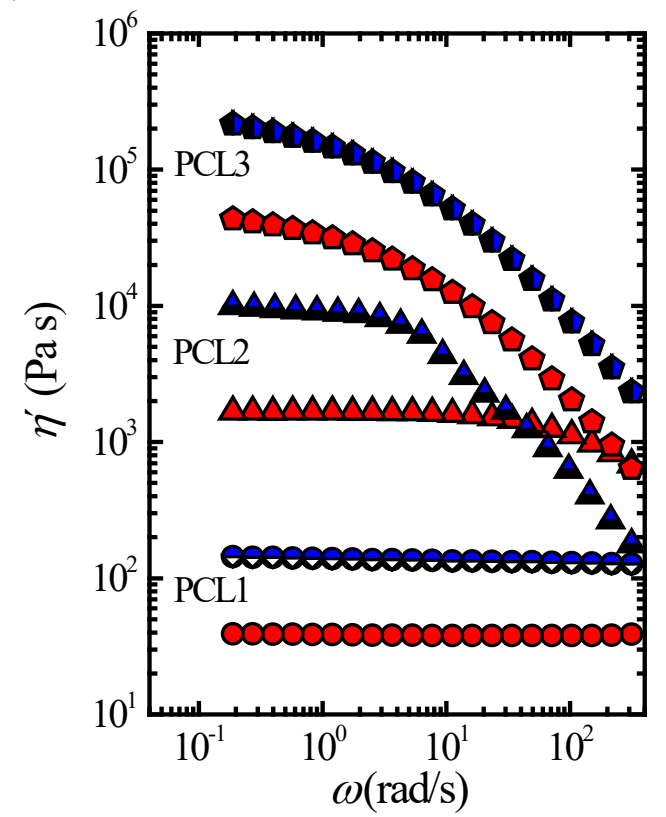

b)

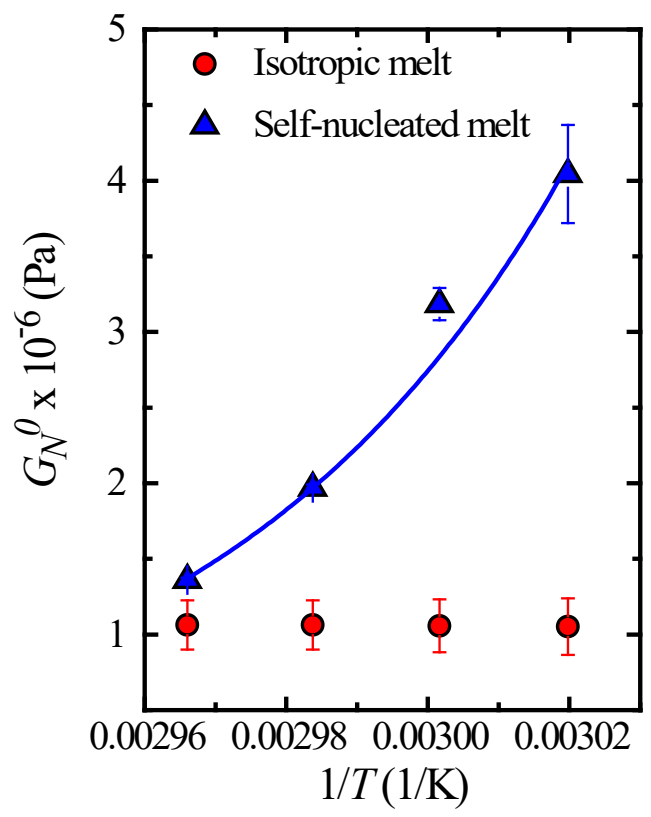

Figure 15. a) Dynamic viscosity $\left(\eta^{\prime}\right)$ as a function of frequency for PCL of different molecular weights. Data are plotted in red for the isotropic melts, and in blue for the self-nucleated melts, the measurements were performed at the same temperature, varying the thermal procedure to obtain different melt states. b) Entanglement plateau modulus as a function of the inverse of the temperature for high 
molecular weight PCL in both the isotropic and self-nucleated melt state ${ }^{97}$. Modified figure from ref. 97. Copyright 2018 Elsevier B.V.

Figure 15 summarizes the results for isotropic and self-nucleated melt states of PCL samples. The dynamic viscosity $\left(\eta^{\prime}\right)$ of self-nucleated PCL, can be up to one order of magnitude higher with respect to that of the isotropic melt, at the same temperature (Figure 15a). The same enhancement was confirmed in samples of different molecular weights. These results are interpreted by considering that self-nuclei are regions of the melt where intermolecular interactions occur, providing additional entanglements, which hinder the diffusion of polymeric chain segments and enhance resistance to flow. The nature of these extra physical entanglements is different with respect to conventional topological entanglements ${ }^{97}$, as they are progressively dissolved by increasing the melt temperature until TDI/DII (see the lower dynamic viscosity values of the isotropic melt).

Figure $15 \mathrm{~b}$ shows the plateau modulus, $G_{N}{ }^{0}$, as a function of the reciprocal measurement temperature for self-nucleated and isotropic PCL melts. The higher entanglement density for the self-nucleated melt is confirmed by the larger value of the modulus, which indicates a denser network ${ }^{97}$. Therefore, while a network of conventional entanglements constitutes the isotropic melt, the self-nucleated melt state consists of a mixed network of conventional entanglements plus new entanglement-like regions arising from the pre-existing interactions of the chain segments within selfnuclei.

Their temperature dependence evidences the different nature of the entanglement network in the two cases. For the isotropic melt state, there is practically no effect of temperature in the plateau modulus in the temperature range analyzed in Figure 15b. However, in the case of the self-nucleated melt, an important reduction of the plateau modulus is observed upon increasing temperature. As expected, increasing temperature progressively destroys the interactions that are present in self-nuclei, and eventually, the SN melt is transformed into an isotropic melt at temperatures above TDI/DII. In fact, at the highest temperature probed in Figure $15 \mathrm{~b}$, the value of the plateau modulus is very close to that of the isotropic melt.

In Figure 15b, the decrease of the plateau modulus, $G_{N}{ }^{0}$, with the inverse temperature is fitted with an exponential equation. It can thus be considered that the decay of the inter-chain interactions is a thermally activated process according to an 
Arrhenius law. An activation energy of about $170 \mathrm{~kJ} / \mathrm{mol}$ for the dissolution of selfnuclei is derived from this rheological method. Such value is in good agreement with previously discussed temperature-dependencies of melt memory effects (see Section $2.2)^{97}$.

We note that the rheological properties of PCL self-nucleated melts are strikingly different from those of isotropic melts, while in the previous investigation of propylene/ethylene copolymers, distinctions between the two states where only found in the thermo-rheological behavior (complexity vs. simplicity) ${ }^{64}$. This observation points toward a stronger melt memory effect in the case of PCL, and it can be tentatively attributed to the stabilization of self-nuclei via additional dipole-dipole interactions in the polyester, with respect to the sole Van Der Waals forces acting in propene/ethylene copolymers $^{65}$.

Since PCL possesses a dipolar moment in the repeating unit, it is possible to study its melt memory effect performing dielectric experiments. As reported in the literature, dielectric measurements are very sensitive to crystallization phenomena, in fact, this technique can detect pre-ordering phenomena, while classical techniques (such as DSC or X ray diffraction) only reveal changes when the crystallization process is in a more advanced stage ${ }^{164-167}$.

In Figure 16, the difference in the normalized dielectric permittivity (i.e., the normalized permittivity of a sample whose thermal history has been erased subtracted by the normalized permittivity of a self-nucleated sample $)^{65}$ is shown for PCLs with two different molecular weights as a function of self-nucleation temperature. The permittivity is normalized with respect to its value at $90^{\circ} \mathrm{C}$, in the isotropic melt.

As it can be seen in Figure 16, the differences in permittivity in Domain II are positive, which indicates that the sample with an isotropic melt has a higher permittivity than the self-nucleated sample. Thus, the presence of the self-nuclei hinders the reorientation of the dipoles with the varying electric field, which results in lower permittivity values. With increasing the temperature, the differences are reduced because the restricted dipoles unlock and become more mobile, contributing to the total permittivity. At temperatures corresponding to Domain I the permittivity of isotropic and SN melts is the same. The number of PCL dipoles involved in the self-nuclei can be roughly estimated by using the Onsager equation. Assuming completely immobile units in self-nucleated melt at $58^{\circ} \mathrm{C}$, a volume fraction of dipoles of the order of $0.4 \%$ was 
derived. Such a remarkably low value can explain the undetectability of self-nuclei by traditional investigation techniques such as X-ray diffraction.

To confirm the close relationship between PCL dipoles with restricted mobility and self-nuclei, a similar parameter based on the crystallization temperature has been defined. In Figure 16b, the difference in the crystallization temperature is shown as a function of temperature. It shows the same trend as the difference in normalized permittivity.

Furthermore, the presence of restricted dipoles in self-nucleated PCL was also found to be associated with the measured increase in Newtonian viscosity of selfnucleated melts with respect to isotropic ones ${ }^{65}$, see Figure 16c. This confirms that measurements of polymer chain mobility in the self-nucleated melt, at various length scales, can be a convenient tool to gain insights into the nature of melt memory effects. 
a)

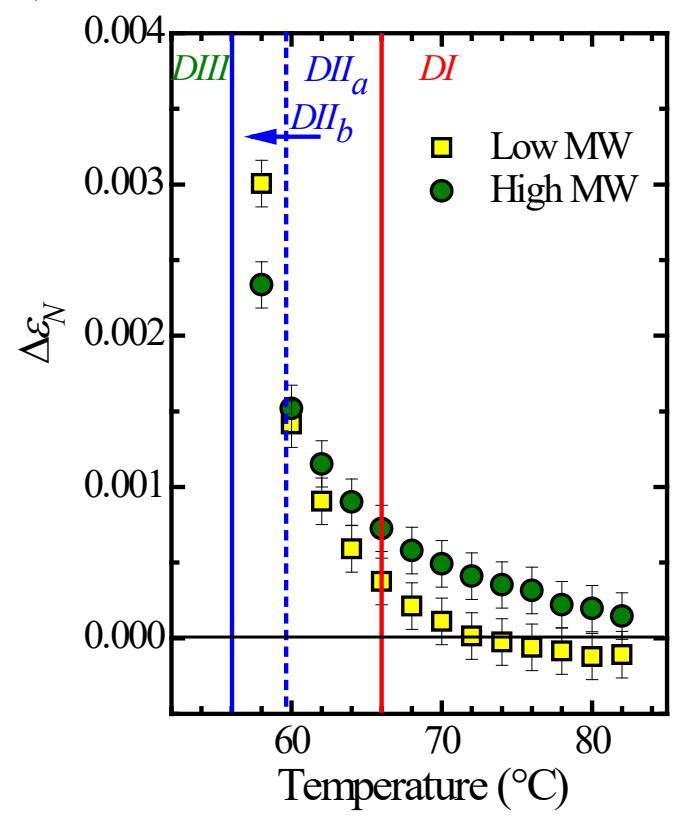

b)

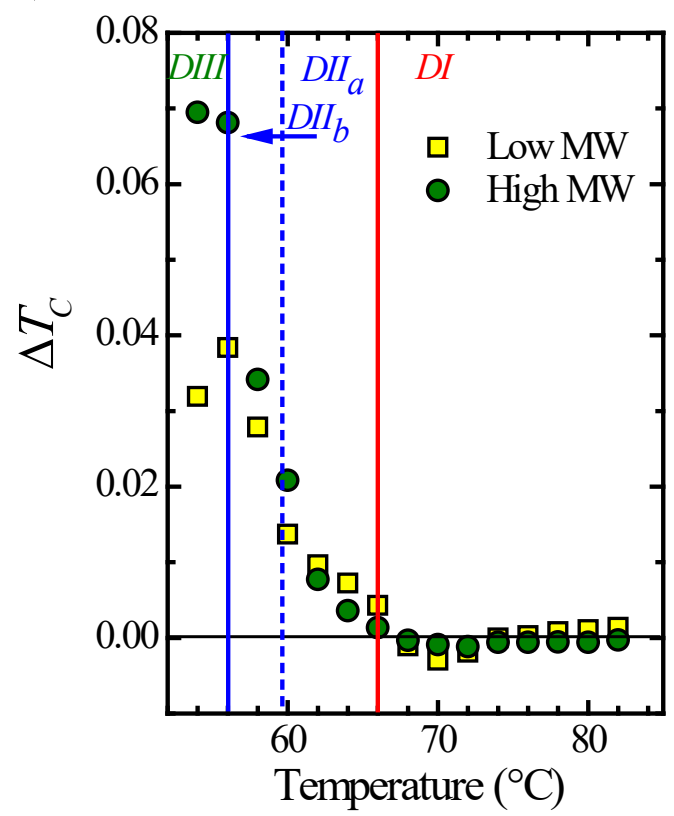

c)

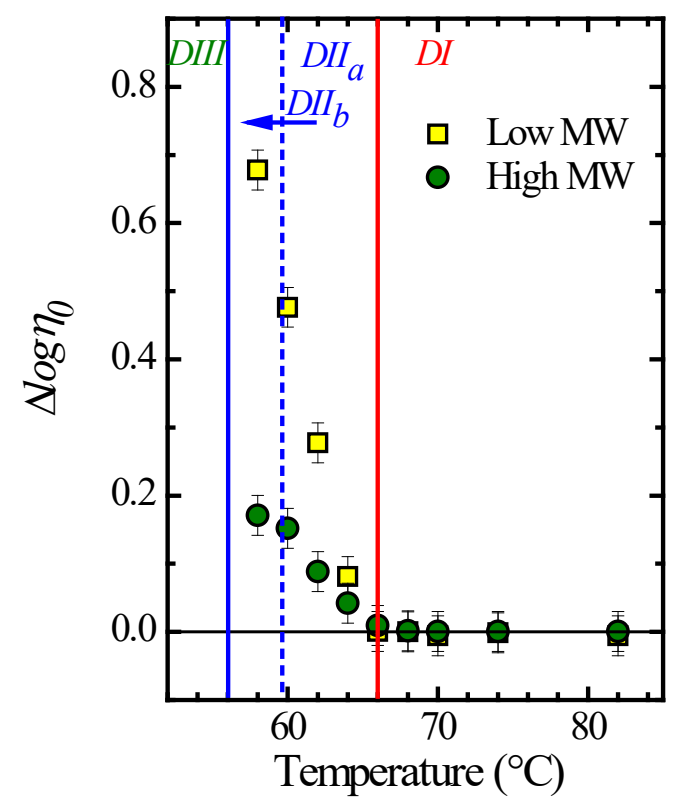

Figure 16. The difference of the normalized a) permittivity $\left(\Delta \varepsilon_{N}=\frac{\varepsilon^{\prime}, \mathrm{IM}-\varepsilon^{\prime}, \mathrm{SNM}}{\varepsilon_{, I M}^{\prime}}\right)$, b) crystallization temperature $\left(\Delta T_{c}=\frac{T_{C, S N M}-T_{C, I M}}{T_{C, I M}}\right)$ and c) viscosity $\left(\Delta \log \eta_{0}=\frac{\log \eta_{0, S N M}-\log \eta_{0, I M}}{\log \eta_{0, I M}\left(90^{\circ} \mathrm{C}\right)}\right)$ for PCL of low and high molecular weights as a function of temperature. Adapted with permission from ref 64 . 
The results obtained by rheology and dielectric relaxation on PCL give support to the idea that self-nuclei are regions of the melt where specific interactions between chain segments exist. The rather strong memory effect of PCL, as compared to polyolefins, which has clear repercussions on melt rheology and permittivity, can be hypothesized to be the result of enhanced inter-molecular interactions. In fact, a recent work, which combined terahertz spectroscopy and quantum chemical calculations, revealed the presence of three different kinds of weak hydrogen bondings between the methylene and carbonyl groups in PCL ${ }^{168}$.

A further demonstration of the role of intermolecular interactions in polymer melt memory effect has been recently obtained for a series of polyamides. As it is well known, polyamide properties are strongly influenced by hydrogen bonding. Liu et al. ${ }^{169}$ have shown with FTIR analysis that the ratio of hydrogen-bonded to free N-H groups in PA1012 in the melt is reduced when the $T_{s}$ temperature is increased. In other words, hydrogen bonding interactions tend to decrease as temperature increases progressively. Therefore, the highest density of hydrogen-bonded groups are obtained in the temperature range corresponding to Domain II. Moreover, by considering the temperature variation of the ratio free/bonded N-H groups, a pseudo-equilibrium constant for hydrogen bonds dissociation could be estimated. The variation of the natural logarithm of this constant is shown as a function of the reciprocal self-nucleation temperature in Figure 17a.

The slope of the plot in Figure 17a is proportional to the enthalpy gain due to chain segmental interactions. The energy required to break the hydrogen bonding interactions is the lowest in the isotropic melt (high temperature range of Domain I), corresponding to ca. $7 \mathrm{~kJ} / \mathrm{mol}$, while it is considerably higher in Domain IIa, where a value of about $75 \mathrm{~kJ} / \mathrm{mol}$ can be calculated. When residual crystal fragments are present (Domain IIb), the strength of intermolecular interaction is even stronger. These results clearly indicate that for this specific polyamide (PA1012), the self-nucleated melt is characterized by persisting hydrogen bonding interactions, which are stronger than those established by the polymer chains in the isotropic melt. 
a)

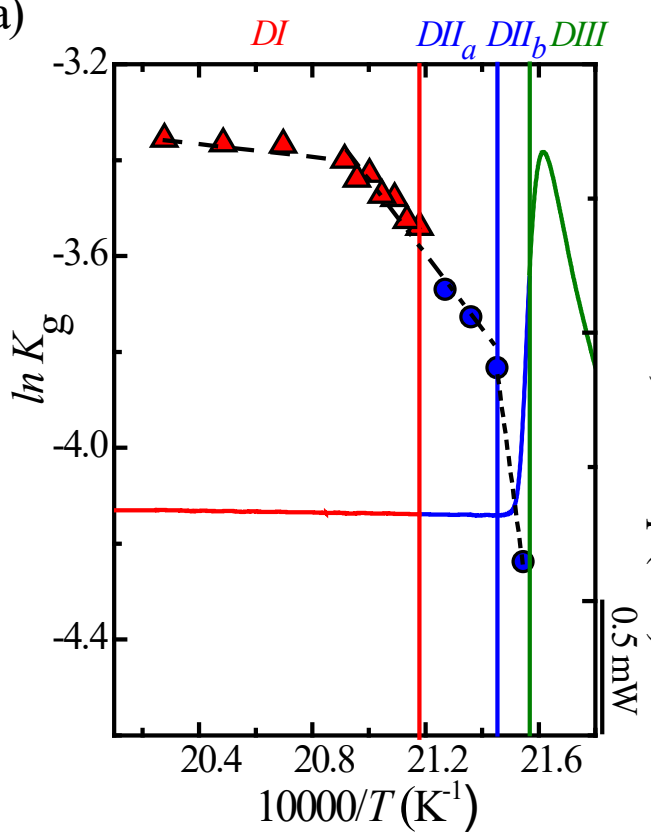

b)

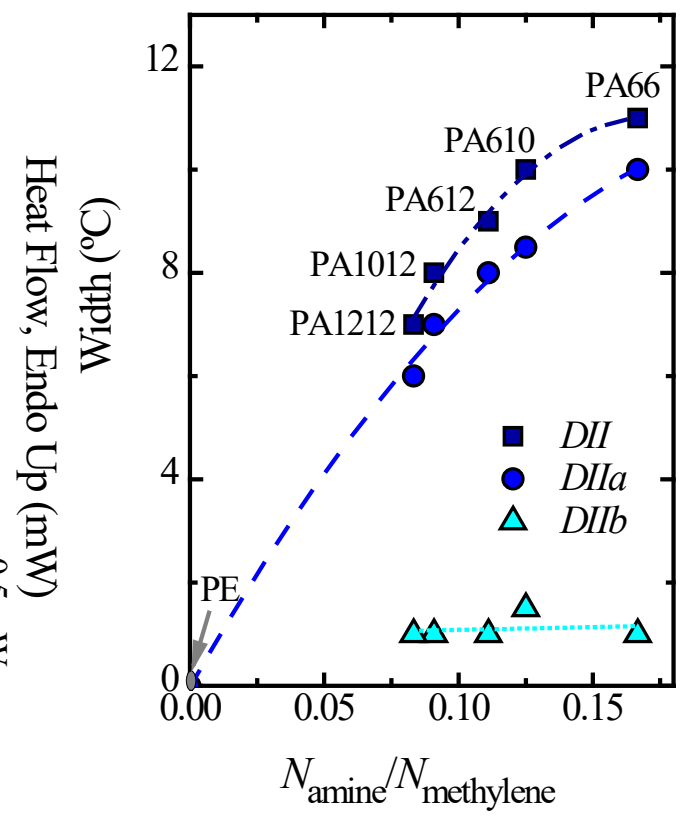

Figure 17. a) Equilibrium constant between hydrogen-bonded and free $\mathrm{N}-\mathrm{H}$ groups as a function of the reciprocal absolute temperature. b) Width of Domain II, Domain IIa and Domain IIb as a function of the ratio of amide to methylene groups in different polyamides with varying content of methylene units in the repeating unit. Modified from ref. 169. Copyright 2020 Elsevier B.V.

Additionally, several different polyamides composed of a varying number of methylene groups in their repeating units were considered, and the different selfnucleation Domains were determined by DSC. Figure $17 \mathrm{~b}$ reports the width of Domain II (also separately including Domain IIa and Domain IIb) as a function of the ratio of the amide to methylene groups in the repeating unit. The results show that the temperature range of Domain IIa increases when the number of methylene groups in the repeating unit is reduced; or, in other words, when the importance of polar (hydrogen bonding) interactions in the polymer increases at the expenses of dispersive (Van Der Waals) forces. On the other hand, the width of Domain IIb, in which small crystal fragments are present, is independent of the amide group density along the polyamide chains. An interesting observation is that Domain IIa width vs. amide/methylene group ratio data can be extrapolated to the origin of the axes. This means that the melt memory effect is expected to disappear for a polymer composed of exclusively methylene groups, a prediction in agreement with the total absence or very limited width of Domain II in polyethylene ${ }^{62,169}$. 
In summary, combining calorimetry with other techniques that can directly probe the differences between self-nucleated and isotropic melts (such as FTIR, rheology and permittivity measurements) provides a way to ascertain the importance of intermolecular interactions between chain segments, which were originally present within the crystalline lamellae. Such intra-crystalline intermolecular interactions seem to be very significant for determining the extent of melt memory effects.

The recent experimental results suggest that the width of Domain IIa, i.e., the persistence of the memory effect, is intimately linked to the strength of such interactions. This apparently trivial but important concept can be further tested on existing literature data. To this aim, an extensive collection of the experimentally determined self-nucleation Domain temperatures for various homopolymers and copolymers is reported in Table S3 of the Supporting Information. To rationalize the data, it is instructive to consider the temperature of the transition between Domain I and Domain II (TDI/DII), which represents the ultimate stability limit of self-nuclei for a given polymer. In Figure 18, this temperature is plotted as a function of the end of the melting endotherm measured by DSC, i.e., the stability limit of the polymer crystallites. The whole set of data can be divided into two classes with distinct behavior, i.e., the homopolymers and the copolymers.

Remarkably, the data for all homopolymers nicely fall on a single line, for a melting temperature range spanning over $200{ }^{\circ} \mathrm{C}$. It can be safely concluded that, as could be expected, the stability of self-nuclei in linear flexible semi-crystalline homopolymers is directly related to the stability of the crystals that originate them. The melting point of a polymer crystal, to a first rough approximation, is determined by the intermolecular forces of the constituent chains. In fact, since the molar entropy of fusion only varies in a narrow range for a wide variety of polymers, and although there might be intramolecular (conformational) contribution to the melting enthalpy ${ }^{170}$, a certain correlation between polymer melting temperature and cohesive energy density has been found ${ }^{171-173}$. Therefore, it seems that the same forces that hold the polymer chains together in the crystals, are responsible for the persistence of melt memory effects in homopolymers.

Concerning the extra-stability of self-nuclei, in comparison with the respective crystals, no clear conclusion can be drawn from the data of Figure 18. In fact, the width of Domain IIa seems unrelated to the melting point of the crystals (see Figure S.1 in the Supporting Information), and the data are scattered around an average value of 
approximately $6^{\circ} \mathrm{C}$. It must be deduced that other factors come into play in determining the "superheating" of self-nuclei with respect to the crystals. These might be related for instance, to specific molecular or morphological features, such as molecular weight or lamellar thickness.

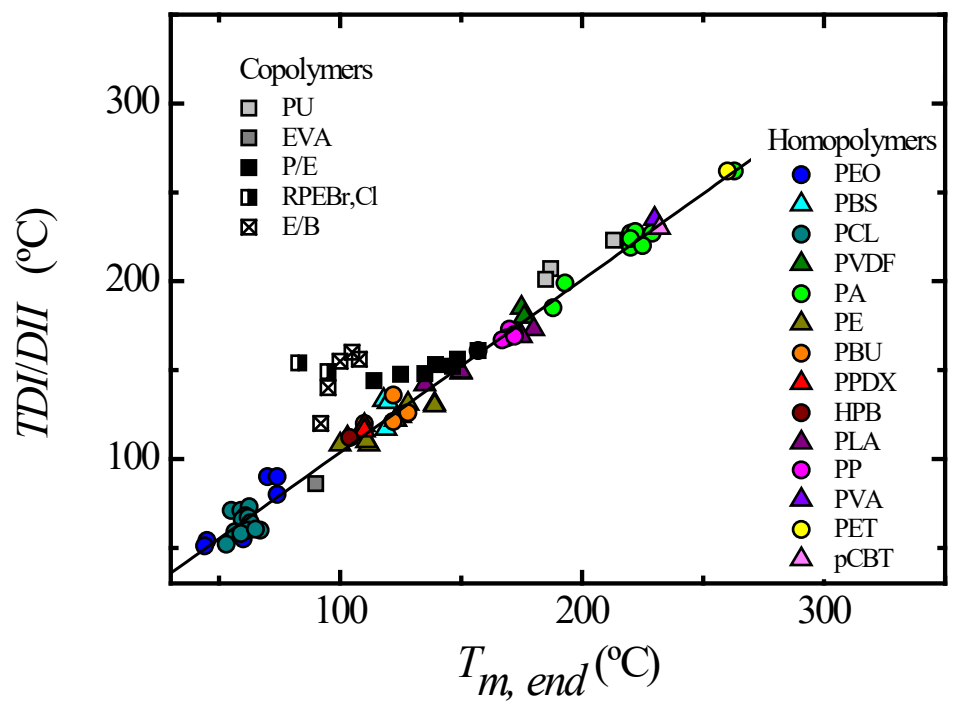

Figure 18. Transition temperature between Domain I and Domain II as a function of the end of the melting temperature for homopolymers and copolymers reported in the literature. The line corresponds to the fitting of the data.

A completely different situation arises for the copolymers. The Domain I/Domain II transition temperatures for these samples lay systematically above the line described by the homopolymer data in Figure 18. In other words, the stability of selfnuclei in these systems is not directly determined by the stability of the parent crystals. The behavior is particularly evident for ethylene/1-butene and ethylene/halogensubstituted ethylene random copolymers, but it is also observed in propene/ethylene copolymers as well as in polyurethanes. As discussed by Alamo and $\mathrm{Hu}$ et al. 52,88,94,110 the melt memory effect of random copolymers has its origin in the complicated topology of the amorphous phase arising from the selection of crystallizable sequences at the crystal growth front. In this respect, the blocky chain structure of polyurethane might be the origin of their intermediate behavior, between that of homopolymers and truly random copolymers. The plot of Figure 18 is thus a strong confirmation of the peculiarity of random copolymer melt memory effect and indicates the direction of further research to improve our understanding of this topic. 


\section{Conclusions}

Since melt memory effect was first detected in the fifties and sixties ${ }^{37-40,66-68,121-}$ ${ }^{127}$, the development of more accurate techniques and more detailed studies, performed with a wide range of polymer systems have allowed shedding light on the parameters that affect it and on its possible nature. In this perspective, the literature findings on the role of the different parameters, such as the effect of molecular weight, chain topology, confinement or holding times in the melt, have been discussed in depth. The different theoretical interpretations, proposed throughout the years to explain the nature of melt memory effects, have also been summarized. While the different concepts which have been put forward might find application in distinct cases, e.g., homopolymers vs. copolymers, a unified model is still lacking. The main obstacle in this respect is the lack of direct probe for the self-nuclei intimate nature, together with the still somehow qualitative predictions, which hamper the validation or rejection of a particular theory.

In our opinion, with the aim of obtaining a general model able to explain all the parameters that affect the melt memory effect, the multi-technique approach should be extended to a wide range of polymeric systems. Until now, only a few works have employed techniques complementary to calorimetry to study self-nucleation ${ }^{61,63-}$ $65,97,106,156$. Rheology, dielectric relaxation and different types of spectroscopy have revealed some details of self-nuclei's nature ${ }^{61,63-65,97}$, as they could detect distinct features in isotropic and self-nucleated melts. Extending these types of studies to several polymeric systems, encompassing homopolymers, copolymers, blends and nanocomposites or confined polymers, seems a promising route to be pursued.

Regarding the effect of homopolymers chemistry on melt memory effects, we have presented a large survey of literature data that highlight the importance of intermolecular interactions. In general, polyolefinic homopolymers, which only display Van der Waals forces, show weak memory effects (i.e., very narrow temperature range of Domain IIa $)^{52,62}$, whereas polymers with polar groups, that have in addition dipoledipole interactions or hydrogen bonding, generally show a broader temperature range in which melt memory effect occurs (broad temperature range of Domain IIa) ${ }^{57,169}$. Nevertheless, systematic experiments on model polymers, whose chemical structure varies in a controlled fashion, are still much needed. Such experiments should reveal more precisely the role of the intermolecular chain segments interactions in the melt memory effect and on the stabilization of the self-nuclei. 
For what concerns apolar random copolymers, the studies carried out with ethylene/1-alkene, ethylene/vinyl halide and propene/ethylene copolymers have shown that the width of the temperature range corresponding to melt memory effects can be increased up to temperatures higher than the equilibrium crystal melting point by inserting a certain amount of comonomers. In this case, the crucial factor for selfnucleation is the complexity of the melt topology ${ }^{52,64,94}$. However, a way of quantifying the extent of complexity in the intercrystalline amorphous phase, which would be of great importance to gain further insights into random copolymers' memory effects, has not been developed yet.

In addition to the above-mentioned issues, several aspects of self-nucleation deserve further investigation. For instance, the reported data on the influence of time on the survival of melt memory, as a function of self-nucleation temperature, also deserves further investigation, since those studies are scarce in literature and not fully consistent. From the few studies available, it can be concluded that if the sample is maintained at temperatures corresponding to Domain IIa (i.e., the temperature region where all crystals have been molten, but where self-nuclei remain active), for very long times, eventually an isotropic melt state can be obtained, especially in the upper end of Domain IIa. On the other hand, if the sample is kept in the temperature range of Domain IIb (i.e., where unmolten crystal fragments remain in the sample), exceedingly long times are needed. In practice, as those long times could result in degradation of the material, it is not possible to observe the complete dissolution of self-seeds and the achievement of the isotropic melt $\mathrm{t}^{43,47,64}$.

Some works which were not covered in detail in this Perspective also raise interesting questions and are worth additional research efforts. It has been recently reported that the presence of impurities can affect melt memory. Men et al. ${ }^{174}$ have shown that in commercial isotactic Poly(butene-1), a melt memory effect could be detected at temperatures higher than the equilibrium melting point. However, when the sample was purified, this peculiar melt memory effect at temperatures higher than the equilibrium melting point disappeared. Therefore, in this case, the melt memory effect at such high temperatures was clearly the result of the presence of impurities. On the one hand this finding highlights the importance of analyzing model materials, which do not contain impurities or additives. On the other hand, they force us to wonder on the role of heterogeneities in memory effects. In fact, adsorption of polymer chains on solid surfaces has been shown to stabilize small but thick crystalline fragments at 
temperatures close to the equilibrium melting point, in the case of poly(butylene terephthalate)/reduced graphene oxide nanocomposites ${ }^{153}$. Moreover, novel experimental results on substrate-polymer interactions show that thin layers of crystalline chains can spontaneously order on a heterogeneous solid surface, even at temperatures above the equilibrium melting point, according to the so-called prewetting transition ${ }^{175,176}$. The relationship between heterogeneities and self-nucleation also needs clarification.

Scarce literature observations have reported faster crystal growth rate upon recrystallization from non-isotropic melts ${ }^{177}$. Since in the majority of cases, the possible effect of melt memory on the growth process is neglected, due to the overwhelming impact on nucleation, it seems worth to re-consider this possibility.

So far, research has been focused on ascertaining the nature or origin of melt memory effects and on the parameters that affect it. However, a practical application to exploit the dramatic nucleation density enhancement that is produced by self-nucleation has not been developed so far, apart from using the $\mathrm{SN}$ technique as an easy way to quantify the efficiency of nucleating agents, as originally proposed by Fillon et al. ${ }^{100}$.

From an industrial point of view, self-nucleation could be highly attractive for two specific purposes: to reduce the time needed to process polymeric materials and to improve its final properties. Employing self-nucleation, the crystallization process can be accelerated up to 2 orders of magnitude, in the case of polymers with a fast crystallization rate $^{118}$. Therefore, the solidification or molding cycles could be shortened, leading to faster processing and thus to more economic production. Another aspect of promoting self-nucleation in a polymeric crystallizable material is that nucleation density can be enhanced from 3 to 7 orders of magnitude. Considering also that with self-nucleation the crystallinity degree of polymers with slow crystallization rates can be increased, properties such as mechanical performance, barrier character, optical properties or the biodegradation rate of biopolymers could also be controlled. Naturally, the relatively low temperatures at which memory effects are observed, presently impair the processing of polymers under ideal self-nucleation conditions. However, with gaining a deeper fundamental understanding, polymers with tailor-made melt topology or crystalline structure could be prepared, possibly enabling technological exploitation of this peculiar phenomenon. 


\section{Supporting Information}

A summary of literature results in nucleation density and spherulite size after the application of SN are shown in Table S1; the variation of the morphology and structure after SN procedure are reported in Table S2; SN results for different materials are listed in Table S3 and the width of DIIa versus the end of melting temperature can be seen in Figure S1.

\section{Acknowledgments}

We acknowledge extensive discussions on melt memory effects with Rufina Alamo, Antxon Santamaria, Giovanni C. Alfonso, Xia Dong, Guoming Liu, Yunlan Su, and Dujin Wang. This work has received funding from the European Union's Horizon 2020 research and innovation program under the Marie Sklodowska-Curie grant agreement No 778092. AJM and LS also acknowledge funding from MINECO, project: MAT2017-83014-C2-1-P and the Basque Government through grant IT1309-19. LS gratefully thanks the Basque Government for her Postdoctoral Fellowship.

\section{References}

(1) Wunderlich, B., Macromolecular Physics, Volume 2: Crystal nucleation, Growth, Annealing; Academic Press: New York, 1973.

(2) Mandelkern, L., Crystallization of Polymers: Volume 2, Kinetics and Mechanisms; Cambridge University Press: Cambridge, 2004

(3) Price F. P. Nucleation in polymer crystallization. In Nucleation; Zettlemoyer, A. C., Ed.; Marcel Dekker: New York, 1969; pp 405-488.

(4) Cormia, R. L.; Price, F. P.; Turnbull, D. Kinetics of crystal nucleation in polyethylene. $J$. Chem. Phys. 1962, 37, 1333-1340.

(5) Sharples, A. The formation of nuclei in crystallizing polymers. Polymer 1962, 3, 250-252.

(6) Sharples, A. Introduction to Polymer Crystallization; Edward Arnold Publishers Ltd.: London, 1966.

(7) Vonnegut, B. Variation with temperature of the nucleation rate of supercooled liquid tin and water drops. J. Colloid Sci. 1948, 3, 563-569.

(8) Turnbull, D.; Cech, R. E. Microscopic observation of the solidification of small metal droplets. J. Appl. Phys. 1950, 21, 804-810.

(9) Pound, G. M.; La Mer, V. K. Kinetics of crystalline nucleus formation in supercooled liquid $\operatorname{Tin}^{1,2}$. J. Am. Chem. Soc. 1952, 74, 2323-2332.

(10) Turnbull, D. Kinetics of solidification of supercooled liquid mercury droplets. J. Chem. Phys. 1952, 20, 411-424.

(11) Turnbull, D.; Cormia, R. L. Kinetics of crystal nucleation in some normal alkane liquids. $J$. Chem. Phys. 1961, 34, 820-831.

(12) Burns, J. R.; Turnbull, D. Kinetics of crystal nucleation in molten isotactic polypropylene. $J$. Appl. Phys. 1966, 37, 4021-4026.

(13) Koutsky, J. A.; Walton, A. G.; Baer, E. Nucleation of polymer droplets. J. Appl. Phys. 1967, $38,1832-1839$.

(14) Gornick, F.; Ross, G. S.; Frolen, L. J. Crystal nucleation in polyethylene: The droplet experiment. J. Polym. Sci. C Polym. Symp. 1967, 18, 79-91.

(15) Barham, P. J.; Jarvis, D. A.; Keller, A. A new look at the crystallization of polyethylene. III. Crystallization from the melt at high supercoolings. J. Polym. Sci. Polym. Phys. Ed. 1982, 20, 1733-1748. 
(16) Massa, M. V.; Carvalho, J. L.; Dalnoki-Veress, K. Direct visualisation of homogeneous and heterogeneous crystallisation in an ensemble of confined domains of poly(ethylene oxide). Eur. Phys. J. E 2003, 12, 111-117.

(17) Massa, M. V.; Dalnoki-Veress, K. Homogeneous crystallization of poly(ethylene oxide) confined to droplets: The dependence of the crystal nucleation rate on length scale and temperature. Phys. Rev. Lett. 2004, 92, 255509.

(18) Massa, M. V.; Lee, M. S. M.; Dalnoki-Veress, K. Crystal nucleation of polymers confined to droplets: Memory effects. J. Polym. Sci. B Polym. Phys. 2005, 43, 3438-3443.

(19) Carvalho, J. L.; Dalnoki-Veress, K. Homogeneous bulk, surface, and edge nucleation in crystalline nanodroplets. Phys. Rev. Lett. 2010, 105, 237801.

(20) Carvalho, J. L.; Dalnoki-Veress, K. Surface nucleation in the crystallisation of polyethylene droplets. Eur. Phys. J. E 2011, 34, 1-6.

(21) Arnal, M. L.; Matos, M. E.; Morales, R. A.; Santana, O. O.; Müller, A. J. Evaluation of the fractionated crystallization of dispersed polyolefins in a polystyrene matrix. Macromol. Chem. Phys. 1998, 199, 2275-2288.

(22) Córdova, M. E.; Lorenzo, A. T.; Müller, A. J.; Gani, L.; Tencé-Girault, S.; Leibler, L. The influence of blend morphology (co-continuous or sub-micrometer droplets dispersions) on the nucleation and crystallization kinetics of double crystalline polyethylene/polyamide blends prepared by reactive extrusion. Macromol. Chem. Phys. 2011, 212, 1335-1350.

(23) Michell, R. M.; Blaszczyk-Lezak, I.; Mijangos, C.; Müller, A. J. Confinement effects on polymer crystallization: From droplets to alumina nanopores. Polymer 2013, 54, 4059-4077.

(24) Van Horn, R. M.; Steffen, M. R.; O'Connor, D. Recent progress in block copolymer crystallization. Polym. Crystallization 2018, e10039.

(25) Loo, Y. L.; Register, R. A. Crystallization within block copolymer mesophases; In Developments in Block Copolymer Science and Technology; Hamley, I. W., Ed.; John Wiley \& Sons Inc.: Chichester, 2004; pp 213-243.

(26) Nandan, B.; Hsu, J. Y.; Chen, H. L. Crystallization behavior of crystalline-amorphous diblock copolymers consisting of a rubbery amorphous block. J. Macromol. Sci. C Polym. Rev. 2006, 46, $143-172$

(27) Takeshita, H.; Shiomi, T.; Takenaka, K.; Arai, F. Crystallization and higher-order structure of multicomponent polymeric systems. Polymer 2013, 54, 4776-4789.

(28) Müller, A. J.; Balsamo, V.; Arnal, M. L.; Jakob, T.; Schmalz, H.; Abetz, V. Homogeneous nucleation and fractionated crystallization in block copolymers. Macromolecules 2002, 35, 3048-3058.

(29) Müller, A. J.; Balsamo, V.; Arnal, M. L. Nucleation and crystallization in diblock and triblock copolymers. Adv. Polym. Sci. 2005, 190, 1-63.

(30) Müller, A. J.; Arnal, M. L.; Trujillo, M.; Lorenzo, A. T. Super-nucleation in nanocomposites and confinement effects on the crystallizable components within block copolymers, miktoarm star copolymers and nanocomposites. Eur. Polym. J. 2011, 47, 614-629.

(31) Müller, A. J.; Arnal, M. L.; Lorenzo, A. T. Crystallization in nano-confined polymeric systems. In Handbook of Polymer Crystallization; Piorkowska, E.; Rutledge, G. C., Eds.; John Wiley and Sons Inc.: Hoboken, 2013; pp 347-372.

(32) Michell, R. M.; Blaszczyk-Lezak, I.; Mijangos, C.; Müller, A. J. Confined crystallization of polymers within anodic aluminum oxide templates. J. Polym. Sci. B Polym. Phys. 2014, 52, 1179-1194.

(33) Michell, R. M.; Müller, A. J. Confined crystallization of polymeric materials. Prog. Polym. Sci. 2016, 54-55, 183-213.

(34) Wu, H.; Higaki, Y.; Takahara, A. Molecular self-assembly of one-dimensional polymer nanostructures in nanopores of anodic alumina oxide templates. Prog. Polym. Sci. 2018, 77, 95-117.

(35) Androsch, R.; Schick, C. Crystal nucleation of polymers at high supercooling of the melt. Adv. Polym. Sci. 2017, 276, 257-288.

(36) Schick, C.; Androsch, R.; Schmelzer, J. W. P. Homogeneous crystal nucleation in polymers. J. Phys. Condens. Matter. 2017, 29, 453002.

(37) Banks, W.; Sharples, A. The effect of melt temperature on nucleation in crystallizing polymers. Macromol. Chem. Phys. 1963, 67, 42-48.

(38) Blundell, D. J.; Keller, A.; Kovacs, A. J. A new self-nucleation phenomenon and its application to the growing of polymer crystals from solution. J. Polym. Sci. B Polym. Lett. 1966, 4, 481486.

(39) Blundell, D. J.; Keller, A. Nature of self-seeding polyethylene crystal nuclei. J. Macromol. Sci. B Phys. 1968, 2, 301-336.

(40) Boon, J.; Challa, G.; Van Krevelen, D. W. Crystallization kinetics of isotactic polystyrene. II. Influence of thermal history on number of nuclei. J. Polym. Sci. A-2 Polym. Phys. 1968, 6, 1835-1851. 
(41) Rault, J.; Robelin, E. Memory effect in liquid polyolefine. Polym. Bull. 1980, 2, 373-381.

(42) Di Filippo, G.; González, M. E.; Gasiba, M. T.; Müller, A. J. Crystalline memory on polycarbonate. J. Appl. Polym. Sci. 1987, 34, 1959-1966.

(43) Fillon, B.; Wittmann, J. C.; Lotz, B.; Thierry, A. Self-nucleation and recrystallization of isotactic polypropylene ( $\alpha$ phase) investigated by differential scanning calorimetry. J. Polym. Sci. B Polym. Phys. 1993, 31, 1383-1393.

(44) Alfonso, G. C.; Scardigli, P. Melt memory effects in polymer crystallization. Macromol. Symp. 1997, 118, 323-328.

(45) Chocinski-Arnault, L.; Gaudefroy, V.; Gacougnolle, J. L.; Rivière, A. Memory effect and crystalline structure in polyamide 11. J. Macromol. Sci. B Phys. 2002, 41, 777-785.

(46) Strobl, G. A thermodynamic multiphase scheme treating polymer crystallization and melting. Eur. Phys. J. E 2005, 18, 295-309.

(47) Lorenzo, A. T.; Arnal, M. L.; Sánchez, J. J.; Müller, A. J. Effect of annealing time on the self-nucleation behavior of semicrystalline polymers. J. Polym. Sci. B Polym. Phys. 2006, 44, 1738-1750.

(48) Maus, A.; Hempel, E.; Thurn-Albrecht, T.; Saalwächter, K. Memory effect in isothermal crystallization of syndiotactic polypropylene -- Role of melt structure and dynamics? Eur. Phys. J. E 2007, 23, 91-101.

(49) Xu, J.; Ma, Y.; Hu, W.; Rehahn, M.; Reiter, G. Cloning polymer single crystals through self-seeding. Nat. Mater. 2009, 8, 348-353.

(50) Balzano, L.; Rastogi, S.; Peters, G. Self-nucleation of polymers with flow: The case of bimodal polyethylene. Macromolecules 2011, 44, 2926-2933.

(51) Cavallo, D.; Gardella, L.; Portale, G.; Müller, A. J.; Alfonso, G. C. On cross- and selfnucleation in seeded crystallization of isotactic poly(1-butene). Polymer 2013, 54, 4637-4644.

(52) Reid, B. O.; Vadlamudi, M.; Mamun, A.; Janani, H.; Gao, H.; Hu, W.; Alamo, R. G. Strong memory effect of crystallization above the equilibrium melting point of random copolymers. Macromolecules 2013, 46, 6485-6497.

(53) Reiter, G. Some unique features of polymer crystallisation. Chem. Soc. Rev. 2014, 43, $2055-$ 2065.

(54) Su, F.; Li, X.; Zhou, W.; Zhu, S.; Ji, Y.; Wang, Z.; Qi, Z., Li, L. Direct formation of isotactic poly(1-butene) form I crystal from memorized ordered melt. Macromolecules 2013, 46, 73997405 .

(55) Luo, C.; Sommer, J.-U. Frozen topology: Entanglements control nucleation and crystallization in polymers. Phys. Rev. Lett. 2014, 112, 195702.

(56) Pérez, R. A.; Córdova, M. E.; López, J. V.; Hoskins, J. N.; Zhang, B.; Grayson, S. M.; Müller, A. J. Nucleation, crystallization, self-nucleation and thermal fractionation of cyclic and linear poly(E-caprolactone)s. React. Funct. Polym. 2014, 80, 71-82.

(57) Arandia, I.; Mugica, A.; Zubitur, M.; Arbe, A.; Liu, G.; Wang, D.; Mincheva, R.; Dubois, P.; Müller, A. J. How composition determines the properties of isodimorphic poly(butylene succinate-ranbutylene azelate) random biobased copolymers: From single to double crystalline random copolymers. Macromolecules 2015, 48, 43-57.

(58) Fernández-d'Arlas, B.; Balko, J.; Baumann, R. P.; Pöselt, E.; Dabbous, R.; Eling, B.; ThurnAlbrecht, T.; Müller, A. J. Tailoring the morphology and melting points of segmented thermoplastic polyurethanes by self-nucleation. Macromolecules 2016, 49, 7952-7964.

(59) Li, X.; Liu, Y.; Tian, X.; Cui, K. Molecular mechanism leading to memory effect of mesomorphic isotactic polypropylene. J. Polym. Sci. B Polym. Phys. 2016, 54, 1573-1580.

(60) Muthukumar, M. Communication: Theory of melt-memory in polymer crystallization. $J$. Chem. Phys. 2016, 145, 031105.

(61) Li, W.; Wu, X.; Chen, X.; Fan, Z. The origin of memory effect in stereocomplex poly (lactic acid) crystallization from melt state. Eur. Polym. J. 2017, 89, 241-248.

(62) Michell, R. M.; Mugica, A.; Zubitur, M.; Müller, A. J. Self-nucleation of crystalline phases within homopolymers, polymer blends, copolymers, and nanocomposites. Adv. Polym. Sci. 2017, 276, $215-256$.

(63) Zhang, H.; Shao, C.; Kong, W.; Wang, Y.; Cao, W.; Liu, C.; Shen, C. Memory effect on the crystallization behavior of poly(lactic acid) probed by infrared spectroscopy. Eur. Polym. J. 2017, 91, $376-385$.

(64) Sangroniz, L.; Cavallo, D.; Santamaria, A.; Müller, A. J.; Alamo, R. G. Thermorheologically complex self-seeded melts of propylene-ethylene copolymers. Macromolecules 2017, 50, 642-651. 
(65) Sangroniz, L.; Alamo, R. G.; Cavallo, D.; Santamaria, A.; Müller, A. J.; Alegría, A. Differences between isotropic and self-nucleated PCL melts detected by dielectric experiments. Macromolecules 2018, 51, 3663-3671.

(66) Blundell, D. J.; Keller, A. Controlled crystal-growing procedures in polyethylene involving self-seeding: Some novel twinning habits. J. Macromol. Sci. B Phys. 1968, 2, 337-359.

(67) Carr, S. H.; Keller, A.; Baer, E. Relationship between self-seeded and epitaxial crystallization from polymer solutions: A potentially new method for molecular weight separation and a new decoration method for alkali halides. J. Polym. Sci. A-2 Polym. Phys. 1970, 8, 1467-1490.

(68) Keller, A.; Willmouth, F. M. Self-seeded crystallization and its potential for molecular weight characterization. I. Experiments on broad distributions. J. Polym. Sci. A-2 Polym. Phys. 1970, 8, $1443-1456$.

(69) Guerra, G.; Vitagliano, V. M.; De Rosa, C.; Petraccone, V.; Corradini, P. Polymorphism in melt crystallized syndiotactic polystyrene samples. Macromolecules 1990, 23, 1539-1544.

(70) Sabino, M. A.; Ronca, G.; Müller, A. J. Heterogeneous nucleation and self-nucleation of poly(p-dioxanone). J. Mater. Sci. 2000, 35, 5071-5084.

(71) Supaphol, P.; Lin, J.-S. Crystalline memory effect in isothermal crystallization of syndiotactic polypropylenes: effect of fusion temperature on crystallization and melting behavior. Polymer 2001, 42, 9617-9626.

(72) Müller, A. J.; Arnal, M. L. Thermal fractionation of polymers. Prog. Polym. Sci. 2005, 30, 559-603.

(73) Wu, M. C.; Woo, E. M. Effects of $\alpha$-form or $\beta$-form nuclei on polymorphic crystalline morphology of poly(butylene adipate). Polym. Int. 2005, 54, 1681-1688.

(74) Sorrentino, A.; Pantani, R.; Titomanlio, G. Kinetics of melting and characterization of the thermodynamic and kinetic properties of syndiotactic polystyrene. J. Polym. Sci. B Polym. Phys. 2007, 45, 196-207.

(75) Sorrentino, A.; Vittoria, V. Chapter 9. Structure, Morphology, and Crystallization Behavior of Syndiotactic Polystyrene; In Syndiotactic Polystyrene: Synthesis, Characterization, Processing, and Applications; Schellenberg, J., Ed.; John Wiley \& Sons, Inc.: Hoboken, 2009; pp 155-193.

(76) Müller, A. J.; Michell, R. M.; Pérez, R. A.; Lorenzo, A. T. Successive self-nucleation and annealing (SSA): Correct design of thermal protocol and applications. Eur. Polym. J. 2015, 65, 132-154.

(77) Zaldua, N.; Liénard, R.; Josse, T.; Zubitur, M.; Mugica, A.; Iturrospe, A.; Arbe, A.; De Winter, J.; Coulembier, O.; Müller, A. J. Influence of chain topology (cyclic versus linear) on the nucleation and isothermal crystallization of poly(L-lactide) and poly(D-lactide). Macromolecules 2018, $51,1718-1732$.

(78) Pérez-Camargo, R. A.; d'Arcy, R.; Iturrospe, A.; Arbe, A.; Tirelli, N.; Müller, A. J. Influence of chain primary structure and topology (branching) on crystallization and thermal properties: The case of polysulfides. Macromolecules 2019, 52, 2093-2104.

(79) Maiz, J.; Fernández-d'Arlas, B.; Li, X.; Balko, J.; Pöselt, E.; Dabbous, R.; Thurn-Albrecht, T.; Müller, A. J. Effects and limits of highly efficient nucleating agents in thermoplastic polyurethane. Polymer 2019, 180, 121676.

(80) Zaldua, N.; Maiz, J.; de la Calle, A.; García-Arrieta, S.; Elizetxea, C.; Harismendy, I.; Tercjak, A.; Müller, A. J. Nucleation and crystallization of PA6 composites prepared by T-RTM: Effects of carbon and glass fiber loading. Polymers 2019, 11, 1680.

(81) Varga, J. Melting memory effect of the $\beta$-modification of polypropylene. J. Thermal Anal. 1986, $31,165-172$.

(82) Häfele, A.; Heck, B.; Hippler, T.; Kawai, T.; Kohn, P.; Strobl, G. Crystallization of poly(ethylene-co-octene): II Melt memory effects on first order kinetics. Eur. Phys. J. E 2005, 16, 217224.

(83) Mamun, A.; Umemoto, S.; Ishihara, N.; Okui, N. Influence of thermal history on primary nucleation and crystal growth rates of isotactic polystyrene. Polymer 2006, 47, 5531-5537.

(84) He, Y.; Xu, Y.; Wei, J.; Fan, Z.; Li, S. Unique crystallization behavior of poly(Llactide)/poly(D-lactide) stereocomplex depending on initial melt states. Polymer 2008, 49, 5670-5675.

(85) Na, B.; Pan, H.; Lv, R.; Zhu, J.; Li, C. A facile route to ordered $\gamma$ phase in poly(vinylidene fluoride) via melt annealing and re-crystallization. Mater. Lett. 2012, 85, 37-39.

(86) Pan, H.; Na, B.; Lv, R.; Li, C.; Zhu, J.; Yu, Z. Polar phase formation in poly(vinylidene fluoride) induced by melt annealing. J. Polym. Sci. B Polym. Phys. 2012, 50, 1433-1437.

(87) Cavallo, D.; Gardella, L.; Portale, G.; Müller, A. J.; Alfonso, G. C. Self-nucleation of isotactic poly(1-butene) in the trigonal modification. Polymer 2014, 55, 137-142. 
(88) Mamun, A.; Chen, X.; Alamo, R. G. Interplay between a strong memory effect of crystallization and liquid-liquid phase separation in melts of broadly distributed ethylene-1-alkene copolymers. Macromolecules 2014, 47, 7958-7970.

(89) Wang, Y.; Lu, Y.; Zhao, J.; Jiang, Z.; Men, Y. Direct formation of different crystalline forms in butene-1/ethylene copolymer via manipulating melt temperature. Macromolecules 2014, 47, $8653-8662$

(90) Chen, X.; Mamun, A.; Alamo, R. G. Effect of level of crystallinity on melt memory above the equilibrium melting temperature in a random ethylene 1-butene copolymer. Macromol. Chem. Phys. 2015, 216, 1220-1226.

(91) Hamad, F. G.; Colby, R. H.; Milner, S. T. Lifetime of flow-induced precursors in isotactic polypropylene. Macromolecules 2015, 48, 7286-7299.

(92) Ren, M.; Chen, X.; Sang, Y.; Alamo, R. G. Effect of heterogeneous short chain branching distribution on acceleration or retardation of the rate of crystallization from melts of ethylene copolymers synthesized with Ziegler-Natta catalysts. Macromol. Symp. 2015, 356, 131-141.

(93) Yin, Y.; Song, Y.; Xiong, Z.; Zhang, X.; de Vos, S.; Wang, R.; Joziasse, C. A. P.; Liu, G.; Wang, D. Effect of the melting temperature on the crystallization behavior of a poly(L-lactide)/poly(Dlactide) equimolar mixture. J. Appl. Polym. Sci. 2016, 133, 43015.

(94) Marxsen, S. F.; Alamo, R. G. Melt-memory of polyethylenes with halogen substitution: Random vs. precise placement. Polymer 2019, 168, 168-177.

(95) Cho, K.; Saheb, D. N.; Choi, J.; Yang, H. Real time in situ X-ray diffraction studies on the melting memory effect in the crystallization of $\beta$-isotactic polypropylene. Polymer 2002, 43, 1407-1416.

(96) Cho, K.; Saheb, D. N.; Yang, H.; Kang, B.-I.; Kim, J.; Lee, S.-S. Memory effect of locally ordered $\alpha$-phase in the melting and phase transformation behavior of $\beta$-isotactic polypropylene. Polymer 2003, 44, 4053-4059.

(97) Sangroniz, L.; Barbieri, F.; Cavallo, D.; Santamaria, A.; Alamo, R. G.; Müller, A. J. Rheology of self-nucleated poly( $\varepsilon$-caprolactone) melts. Eur. Polym. J. 2018, 99, 495-503.

(98) Fenni, S. E.; Wang, J.; Haddaoui, N.; Favis, B. D.; Müller, A. J.; Cavallo, D. Crystallization and self-nucleation of PLA, PBS and PCL in their immiscible binary and ternary blends. Thermochim. Acta 2019, 677, 117-130.

(99) Corradini, P.; Guerra, G. Polymorphism in polymers. Adv. Polym. Sci. 1992, 100, 183-217.

(100) Fillon, B.; Lotz, B.; Thierry, A.; Wittmann, J. C. Self-nucleation and enhanced nucleation of polymers. Definition of a convenient calorimetric "efficiency scale" and evaluation of nucleating additives in isotactic polypropylene ( $\alpha$ phase). J. Polym. Sci. B Polym. Phys. 1993, 31, 1395-1405.

(101) Fillon, B.; Thierry, A.; Wittmann, J. C.; Lotz, B. Self-nucleation and recrystallization of polymers. Isotactic polypropylene, $\beta$ phase: $\beta$ - $\alpha$ conversion and $\beta$ - $\alpha$ growth transitions. J. Polym. Sci. B Polym. Phys. 1993, 31, 1407-1424.

(102) Ho, R.-M.; Lin, C.-P.; Tsai, H.-Y.; Woo, E.-M. Metastability studies of syndiotactic polystyrene polymorphism. Macromolecules 2000, 33, 6517-6526.

(103) Schneider, S.; Drujon, X.; Lotz, B.; Wittmann, J. C. Self-nucleation and enhanced nucleation of polyvinylidene fluoride ( $\alpha$-phase). Polymer 2001, 42, 8787-8798.

(104) Zhu, X.; Li, Y.; Yan, D.; Fang, Y. Crystallization behavior of partially melting isotactic polypropylene. Polymer 2001, 42, 9217-9222.

(105) De Rosa, C.; Ruiz de Ballesteros, O.; Di Gennaro, M.; Auriemma, F. Crystallization from the melt of $\alpha$ and $\beta$ forms of syndiotactic polystyrene. Polymer 2003, 44, 1861-1870.

(106) Mamun, A.; Umemoto, S.; Okui, N. Self-seeding effect on primary nucleation of isotactic polystyrene. Macromolecules 2007, 40, 6296-6303.

(107) Pan, P.; Zhao, L.; Zhu, B.; He, Y.; Inoue, Y. Fractionated crystallization and selfnucleation behavior of poly(ethylene oxide) in its miscible blends with poly(3-hydroxybutyrate). J. Appl. Polym. Sci. 2010, 117, 3013-3022.

(108) Zhou, T. N.; Yang, H.; Ning, N.-Y.; Xiang, Y.-F.; Du, R.-N.; Fu, Q. Partial melting and recrystallization of isotactic polypropylene. Chinese J. Polym. Sci. 2010, 28, 77-83.

(109) Zhang, Y.-S.; Zhong, L.-W.; Yang, S.; Liang, D.-H.; Chen, E.-Q. Memory effect on solution crystallization of high molecular weight poly(ethylene oxide). Polymer 2012, 53, 3621-3628.

(110) Gao, H.; Vadlamudi, M.; Alamo, R. G.; Hu, W. Monte Carlo simulations of strong memory effect of crystallization in random copolymers. Macromolecules 2013, 46, 6498-6506.

(111) Li, X.; Su, F.; Ji, Y.; Tian, N.; Lu, J.; Wang, Z.; Qi, Z.; Li, L. Influence of the memory effect of a mesomorphic isotactic polypropylene melt on crystallization behavior. Soft Matter 2013, 9 , $8579-8588$. 
(112) Kang, J.; Chen, Z.; Zhou, T.; Yang, F.; Chen, J.; Cao, Y.; Xiang, M. Dynamic crystallization and melting behavior of $\beta$-nucleated isotactic polypropylene with different melt structures. J. Polym. Res. 2014, 21, 384.

(113) Kang, J.; Zhang, J.; Chen, Z.; Yang, F.; Chen, J.; Cao, Y.; Xiang, M. Isothermal crystallization behavior of $\beta$-nucleated isotactic polypropylene with different melt structures. J. Polym. Res. 2014, 21, 506.

(114) Kang, J.; Weng, G.; Chen, Z.; Chen, J.; Cao, Y.; Yang, F.; Xiang, M. New understanding in the influence of melt structure and $\beta$-nucleating agents on the polymorphic behavior of isotactic polypropylene. RSC Adv. 2014, 4, 29514-29526.

(115) Guan, X.; Gao, H.; Zha, L.; Wu, Y.; Hu, W. Strong memory of strain-induced copolymer crystallization as revealed by Monte Carlo simulations. Polymer 2016, 98, 282-286.

(116) Hu, D.-D.; Ye, S.-B.; Yu, F.; Feng, J.-C. Further understanding on the three domains of isotactic polypropylene by investigating the crystalline morphologies evolution after treatment at different domains. Chinese J. Polym. Sci. 2016, 34, 344-358.

(117) Luo, C.; Sommer, J.-U. Role of thermal history and entanglement related thickness selection in polymer crystallization. ACS Macro Lett. 2016, 5, 30-34.

(118) Jiang, J.; Zhuravlev, E.; Hu, W.-B.; Schick, C.; Zhou, D.-S. The effect of self-nucleation on isothermal crystallization kinetics of poly(butylene succinate) (PBS) investigated by differential fast scanning calorimetry. Chinese J. Polym. Sci. 2017, 35, 1009-1019.

(119) Zheng, Y.; Zhang, J.; Sun, X.; Li, H.; Ren, Z.; Yan, Z. Crystal structure regulation of ferroelectric poly(vinylidene fluoride) via controlled melt-recrystallization. Ind. Eng. Chem. Res. 2017, $56,4580-4587$.

(120) Cavallo, D.; Zhang, L.; Sics, I.; Alfonso, G. C.; Dumas, P.; Marco, C.; Ellis, G. The morphology and polymorphism of self-nucleated trigonal isotactic poly(1-butene) studied by synchrotron IR microspectroscopy. CrystEngComm 2016, 18, 816-828.

(121) Hawkins, S. W.; Richards, R. B. Light transmission and the formation and decay of spherulites in polythene. J. Polym. Sci. 1949, 4, 515-522.

(122) Price, F. P. The development of crystallinity in polychlorotrifluoroethylene. J. Am. Chem. Soc. 1952, 74, 311-318.

(123) Rabesiaka, J.; Kovacs, A. J. Isothermal crystallization kinetics of polyethylene. III. Influence of the sample preparation. J. Appl. Phys. 1961, 32, 2314-2320.

(124) Magill, J. H. Melting behaviour and spherulitic crystallization of polycaproamide (nylon 6). Polymer 1962, 3, 43-51.

(125) McLaren, J. V. A kinetic study of the isothermal spherulitic crystallization of polyhexamethylene adipamide. Polymer 1963, 4, 175-189.

(126) Schultz, J. M. Transient effects in the crystallization of polyethylene. J. Polym. Sci. A-2 Polym. Phys. 1969, 7, 821-827.

(127) Vidotto, G.; Lévy, D.; Kovacs, A. J. Cristallisation et fusion des polymères autoensemencés. Kolloid Z Z Polymere 1969, 230, 289-305.

(128) Bassett, D. C. Principles of Polymer Morphology; Cambridge University Press: Cambridge, 1981.

(129) Androsch, R.; Zhang, R.; Schick, C. Melt-recrystallization of poly (1-lactic acid) initially containing $\alpha^{\prime}$-crystals. Polymer 2019, 176, 227-235.

(130) Jariyavidyanont, K.; Androsch, R.; Schick, C. Crystal reorganization of poly (butylene terephthalate). Polymer 2017, 124, 274-283.

(131) Androsch, R.; Schick, C.; Rhoades, A. M. Application of Tammann's two-stage crystal nuclei development method for analysis of the thermal stability of homogeneous crystal nuclei of poly (ethylene terephthalate). Macromolecules 2015, 48, 8082-808.

(132) Tang, X.; Chen, W.; Li, L. The tough journey of polymer crystallization: Battling with chain flexibility and connectivity. Macromolecules 2019, 52, 3575-3591.

(133) Janeschitz-Kriegl, H.; Eder, G. Shear induced crystallization, a relaxation phenomenon in polymer melts: A re-collection. J. Macromol. Sci. B Phys. 2007, 46, 591-601.

(134) Galeski, A.; Psarski, M. Morphology and kinetics of crystallization of polyethylene from chain disentangled melt. Macromol. Symp. 1996, 104, 183-190.

(135) Rastogi, S.; Lippits, D. R.; Peters, G. W. M.; Graf, R.; Yao, Y.; Spiess, H. W. Heterogeneity in polymer melts from melting of polymer crystals. Nat. Mater. 2005, 4, 635-641.

(136) Pawlak, A.; Krajenta, J.; Galeski, A. The crystallization of polypropylene with reduced density of entanglements. J. Polym. Sci. B Polym. Phys. 2017, 55, 748-756.

(137) Schick, C.; Androsch, R. Nucleation-controlled semicrystalline morphology of bulk polymers. Polym. Crystallization 2018, 1, e10036. 
(138) Wang, Y.; Liu, P.; lu, Y.; Men, Y. Mechanism of polymorph selection during crystallization of random butene-1/ethylene copolymer. Chinese J. Polym. Sci. 2016, 34, 1014-1020.

(139) Zhao, J.; Sun, Y.; Men, Y. Melt temperature and initial polymorphs dependencies of polymorphs selection during subsequent crystallization in propylene-ethylene random copolymer. Ind. Eng. Chem. Res. 2017, 56, 198-205.

(140) Lai, Y.; Men, Y. Polymorph selection during crystallization of random copolymers. Eur. Polym. J. 2018, 101, 218-224.

(141) Hu, C.; Li, J.; Huang, S.; Li, H.; Chen, J.; Yu, D.; de Claville Christiansen, J.; Jiang, S.; An, L. Memory effects on crystallization behaviours of poly(L-lactic acid) revisited. CrystEngComm 2019, 21, 2660-2668.

(142) Alfonso, G. C.; Ziabicki, A. Memory effects in isothermal crystallization II. Isotactic polypropylene. Colloid Polym. Sci. 1995, 273, 317-323.

(143) Chen, X.; Qu, C.; Alamo, R. G. Effect of annealing time and molecular weight on melt memory of random ethylene 1-butene copolymers. Polym. Int. 2019, 68, 248-256.

(144) Cavallo, D.; Azzurri, F.; Balzano, L.; Funari, S. S.; Alfonso, G. C. Flow memory and stability of shear-induced nucleation precursors in isotactic polypropylene. Macromolecules 2010, 43, 9394-9400.

(145) Azzurri, F.; Alfonso, G. C. Insights into formation and relaxation of shear-induced nucleation precursors in isotactic polystyrene. Macromolecules 2008, 41, 1377-1383.

(146) Chen, X.; Wignall, G. D.; He, L.; Lopez-Barron, C.; Alamo, R. G. SANS evidence of liquid-liquid phase separation leading to inversion of crystallization rate of broadly distributed random ethylene copolymers. Macromolecules 2017, 50, 4406-4414.

(147) Balsamo, V.; Müller, A. J.; Stadler, R. Antinucleation effect of the polyethylene block on the polycaprolactone block in ABC triblock copolymers. Macromolecules 1998, 31, 7756-7763.

(148) Balsamo, V.; Paolini, Y.; Ronca, G.; Müller, A. J. Crystallization of the polyethylene block in polystyrene-b-polyethylene-b-polycaprolactone triblock copolymers, 1. Self-nucleation behavior. Macromol. Chem. Phys. 2000, 201, 2711-2720.

(149) Schmalz, H.; Müller, A. J.; Abetz, V. Crystallization in ABC triblock copolymers with two different crystalline end blocks: Influence of confinement on self-nucleation behaviour. Macromol. Chem. Phys. 2003, 204, 111-124.

(150) Müller, A. J.; Albuerne, J.; Marquez, L.; Raquez, J.-M.; Degée, P.; Dubois, P.; Hobbs, J.; Hamley, I. W. Self-nucleation and crystallization kinetics of double crystalline poly(p-dioxanone)-bpoly(E-caprolactone) diblock copolymers. Faraday Discuss. 2005, 128, 231-252.

(151) Arnal, M. L.; López-Carrasquero, F.; Laredo, E.; Müller, A. J. Coincident or sequential crystallization of PCL and PEO blocks within polystyrene-b-poly(ethylene oxide)-b-poly( $\varepsilon$-caprolactone) linear triblock copolymers. Eur. Polym. J. 2004, 40, 1461-1476.

(152) Trujillo, M.; Arnal, M. L.; Müller, A. J.; Laredo, E.; Bredeau, S.; Bonduel, D.; Dubois, P. Thermal and morphological characterization of nanocomposites prepared by in-situ polymerization of high-density polyethylene on carbon nanotubes. Macromolecules 2007, 40, 6268-6276.

(153) Colonna, S.; Pérez-Camargo, R. A.; Chen, H.; Liu, G.; Wang, D.; Müller, A. J.; Saracco, G.; Fina, A. Supernucleation and orientation of poly(butylene terephthalate) crystals in nanocomposites containing highly reduced graphene oxide. Macromolecules 2017, 50, 9380-9393.

(154) Wen, X.; Su, Y.; Shui, Y.; Zhao, W.; Müller, A. J.; Wang, D. Correlation between grafting density and confined crystallization behavior of poly(ethylene glycol) grafted to silica. Macromolecules 2019, 52, 1505-1516.

(155) Zhu, X.; Yan, D.; Yao, H.; Zhu, P. In situ FTIR spectroscopic study of the regularity bands and partial-order melts of isotactic poly(propylene). Macromol. Rapid Commun. 2000, 21, 354-357.

(156) Zheng, C.; Zhang, X.; Dong, X.; Zhao, Y.; Wang, Z.; Zhu, S.; Xu, D.; Wang, D. Variations of regular conformation structures in melt of syndiotactic polypropylene. Polymer 2006, 47, 7813-7820.

(157) Gurarslan, A.; Joijode, A. S.; Tonelli, A. E. Polymers coalesced from their cyclodextrin inclusion complexes: What can they tell us about the morphology of melt crystallized polymers? $J$. Polym. Sci. B Pol. Phys. 2012, 50, 813-823.

(158) Tonelli, A. E. Enhancing the melt crystallization of polymers, especially slow crystallizing polymers like PLLA and PET. Polym. Crystallization 2019, e10095.

(159) Ziabicki, A.; Alfonso G. C.; Memory effects in isothermal crystallization. I. Theory. Colloid Polym. Sci. 1994, 272, 1027-1042.

(160) Lorenzo, A. T.; Müller, A. J. Estimation of the nucleation and crystal growth contributions to the overall crystallization energy barrier. J. Polym. Sci. B Polym. Phys. 2008, 46, 1478-1487.

(161) Bremner, T.; Rudin, A. Persistence of regions with high segment density in polyethylene melts. J. Polym. Sci. B Polym. Phys. 1992, 30, 1247-1260. 
(162) Blom, H. P.; Teh, J. W.; Bremner, T.; Rudin, A. Isothermal and non-isothermal crystallization of PP: effect of annealing and of the addition of HDPE. Polymer 1998, 39, 4011-4022.

(163) Lippits, D. R.; Rastogi, S.; Höhne, G. W. H.; Mezari, B.; Magusin, P. C. M. M. Heterogeneous distribution of entanglements in the polymer melt and its influence on crystallization. Macromolecules 2007, 40, 1004-1010.

(164) Wurm, A.; Soliman, R.; Schick, C. Early stages of polymer crystallization-a dielectric study. Polymer 2003, 44, 7467-7476.

(165) Wurm, A.; Soliman, R.; Goossens, J. G. P.; Bras, W.; Schick, C. Evidence of precrystalline-order in supercooled polymer melts revealed from simultaneous dielectric spectroscopy and SAXS. J. Non-Cryst. Solids 2005, 351, 2773-2779.

(166) Wurm, A.; Minakov, A.; Schick, C. Combining X-ray scattering with dielectric and calorimetric experiments for monitoring polymer crystallization. Eur. Polym. J. 2009, 45, 3282-3291.

(167) Soccio, M.; Nogales, A.; Lotti, N.; Munari, A.; Ezquerra, T. A. Evidence of early stage precursors of polymer crystals by dielectric spectroscopy. Phys. Rev. Lett. 2007, 98, 037801.

(168) Funaki, C.; Yamamoto, S.; Hoshina, H.; Ozaki, Y.; Sato, H. Three different kinds of weak $\mathrm{C}-\mathrm{H} \cdots \mathrm{O}=\mathrm{C}$ inter- and intramolecular interactions in poly $(\varepsilon$-caprolactone $)$ studied by using terahertz spectroscopy, infrared spectroscopy and quantum chemical calculations. Polymer 2018, 137, 245-254.

(169) Liu, X.; Wang, Y.; Wang, Z.; Cavallo, D.; Müller, A. J.; Zhu, P.; Zhao, Y.; Dong, X.; Wang, D. The origin of memory effects in the crystallization of polyamides: Role of hydrogen bonding. Polymer 2020, 122117, in press.

(170) Wunderlich, B. Macromolecular Physics Volume 3: Crystal melting; Academic Press: New York, 1980.

(171) Bunn, C. W. The melting points of chain polymers. J. Polym. Sci. B Polym. Phys. 1996, $34,799-819$.

(172) Van Krevelen, D. W. Properties of Polymers; Elsevier: Amsterdam, 1972.

(173) Askadskii, A. A., Matveev, Y. I.; Slonimskii, G. L., Korschk, V. V., Dokl.Akad. Nauk. Effect of inter-molecular interaction energy of different types of bonds on temperature of polymer melting. SSSR 1978, 238, 592.

(174) Liu, P.; Xue, Y.; Men, Y. Melt memory effect beyond the equilibrium melting point in commercial isotactic polybutene-1. Ind. Eng. Chem. Res. 2019, 58, 5472-5478.

(175) Flieger, A.-K.; Schulz, M.; Thurn-Albrecht, T. Interface-induced crystallization of polycaprolactone on graphite via first-order prewetting of the crystalline phase. Macromolecules 2018, 51, 189-194.

(176) Tariq, M.; Dolynchuk, O.; Thurn-Albrecht, T. Effect of substrate interaction on thermodynamics of prefreezing. Macromolecules 2019, 52, 9140-9148.

(177) Lu, J.; Yang, H.; Ji, Y.; Li, X.; Lv, Y.; Su, F.; Li, L. Strong memory effect of metastable $\beta$ form trans-1,4-polyisoprene above equilibrium melting temperature. Macromol. Chem. Phys. 2017, 218, 1700235 . 
For Table of Contents use only:

\title{
Memory Effects on Polymer Crystallization
}

\author{
Leire Sangroniz ${ }^{1}$, Dario Cavallo ${ }^{2 *}$ and Alejandro J. Müller ${ }^{1,3 *}$
}

${ }^{1}$ POLYMAT and Polymer Science and Technology Department, Faculty of Chemistry, University of the Basque Country UPV/EHU, Paseo Manuel de Lardizábal, 3, 20018 Donostia-San Sebastián, Spain.

${ }^{2}$ Department of Chemistry and Industrial Chemistry, University of Genova, via Dodecaneso, 31 - 16146 Genova, Italy

${ }^{3}$ IKERBASQUE, Basque Foundation for Science, Bilbao, Spain.






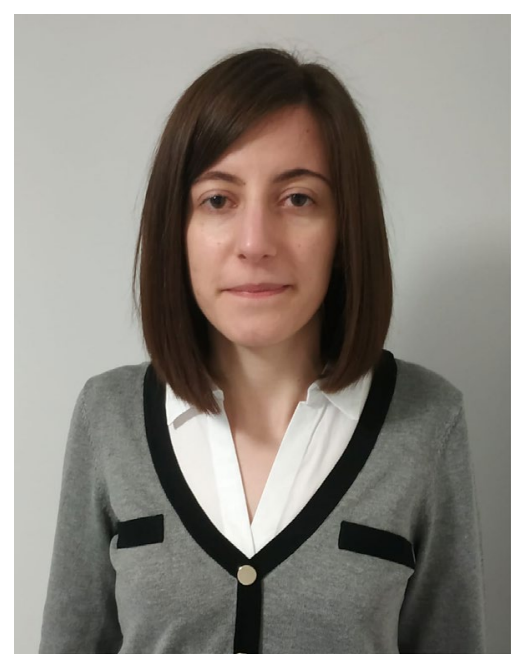

Leire Sangroniz earned her B.Sc. in Chemistry in 2014 and M.Sc. in Applied Chemistry and Polymeric Materials in 2015 from the University of the Basque Country UPV/EHU in Donostia- San Sebastián (Spain). She obtained her Ph.D. from the same university in Applied Chemistry and Polymeric Materials in 2019 (Advisors: Antxon Santamaria and Alejandro J. Müller). She is actually a Postdoctoral Fellow at the University of the Basque Country. Her research is focused on the development of complex multiphasic polymeric systems, including recycled and biodegradable polymers, and on the investigation of their rheology, crystallization and physical properties. 


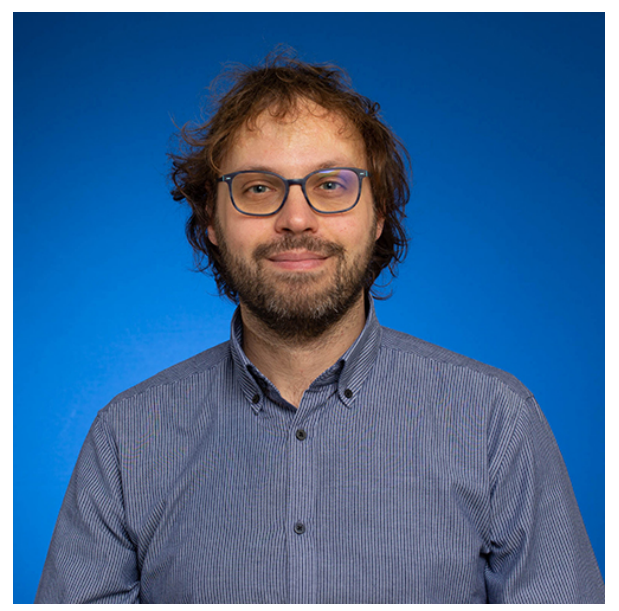

Dario Cavallo graduated in Industrial Chemistry at the University of Genova in 2007. Afterwards he achieved the Ph.D. title in Chemical Sciences from the same University in 2011, studying polymer crystallization in "processing-relevant" conditions (supervisor: Prof. Giovanni C. Alfonso). After the achievement of the Ph.D., he spent few months as a visiting researcher in Madrid (Spain) at the Institute of Polymer Science and Technology (ICTP-CSIC), applying infrared spectroscopy to polymorphic polymers. Subsequently (2011-2013), he was employed in a post-doc position at the Eindhoven University of Technology (The Netherlands), conducting research on structure-properties relationship in semicrystalline polymers for the Dutch Polymer Institute. Since July 2013 he became Assistant Professor at the Department of Chemistry and Industrial Chemistry of the University of Genoa. His main research topics are related to the kinetics of polymer crystallization, concerning in particular the role of solidification conditions and molecular features. In this framework, he is interested in various aspects of primary nucleation (including memory effects and heterogeneous nucleation at various surfaces), polymorphism, crystallization of copolymers and immiscible blends and structuring under processing conditions. The results of his research have been published in about 70 scientific publications. Since 2018 he is member of the Editorial Board of the journal "Polymer Crystallization" and of the International Advisory Board of "Chinese Journal of Polymer Science". 


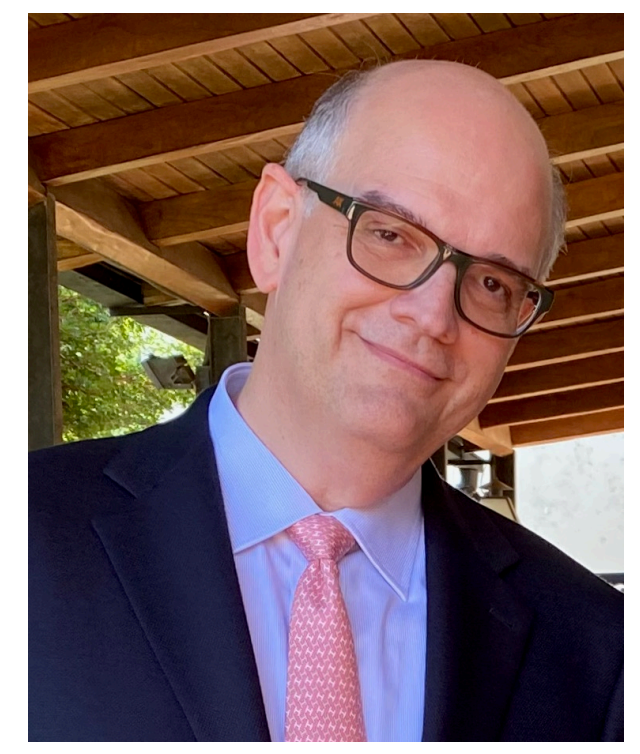

Alejandro J. Müller is a Materials Engineer (Simón Bolívar University, Caracas, Venezuela), with an M.Sc. in Chemistry (Venezuelan Institute for Scientific Research, IVIC, Caracas, Venezuela) and a Ph.D. in Physics (Bristol University, U.K., advisors: Andrew Keller and Jeff Odell). He worked for nearly 30 years as a professor at the Materials Science Department of Simón Bolívar University in Caracas, Venezuela, where he founded and coordinated a very productive research group in Polymer Science. He became a Corresponding Member of the "Academia Nacional de la Ingeniería y del Hábitat de Venezuela (ANIH)" or National Academy of Engineering and Habitat from Venezuela. He won several awards in Venezuela, including the Lorenzo Mendoza Fleury, Polar Prize for basic science (in Chemistry). In 2011 he received the international "Paul J. Flory Polymer Research Prize" for his contributions on the research of Confined Crystallization. Since September 2013, he holds an IKERBASQUE (Basque Foundation for Science) Research Chair at POLYMAT and the Polymer Science and Technology Department, Faculty of Chemistry, University of the Basque Country UPV/EHU in Donostia-San Sebastián, Spain, where he leads the Advanced Multiphasic Polymers Group. He is an Editor for POLYMER (Elsevier) in the joint areas of Polymer Physics and Physical Chemistry. His fields of interest include structure, morphology, nucleation, crystallization, crystallization kinetics, rheology and properties of multiphasic and confined polymeric materials (in particular block copolymers, random copolymers, nano-composites, hybrids and polymer blends). 